\title{
THE $q$-ANALOGUE OF THE WILD FUNDAMENTAL GROUP AND THE INVERSE PROBLEM OF THE GALOIS THEORY OF $q$-DIFFERENCE EQUATIONS
}

\author{
by
}

Jean-Pierre Ramis \& Jacques Sauloy

\begin{abstract}
In 20, 21, we defined $q$-analogues of alien derivations for linear analytic $q$-difference equations with integral slopes and proved a density theorem (in the Galois group) and a freeness theorem. In this paper, we completely describe the wild fundamental group and apply this result to the inverse problem in $q$-difference Galois theory.
\end{abstract}

\section{Contents}

1. Introduction..................................

2. Previous results on the structure of the local Galois group 8

3. Structure of the Stokes component................ 24

4. Structure of the global Galois group................ 36

5. The inverse problem ........................... 42

6. The local inverse problem...................... 52

7. About the global inverse problem................... 64

Appendix A. Pronilpotent completions.................. 67

References................................... 70

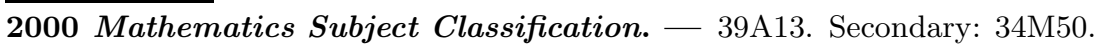

Key words and phrases. - q-difference equations, Stokes phenomenon, alien derivations, Galois theory, inverse problem. 


\section{Introduction}

1.1. The problems. - The main purpose of this paper is to give a new and probably definitive version of the local meromorphic classification of $q$ difference modules in the integral slopes case(1). Using this result we shall get a complete solution of the inverse problem for the $q$-difference Galois theory in the local case, for all $q \in \mathbf{C}^{*},|q| \neq 1$, and a solution of the inverse problem for connected reductive algebraic groups in the global case, also for all $q \in \mathbf{C}^{*}$, $|q| \neq 1$ (for the case of the exceptional simple groups, in particular, this result is new $(2)$.

1.1.1. The q-wild fundamental group. - In [22] we gave three versions of the local meromorphic classification of $q$-difference modules (in the integral slopes case). The first one uses algebraic normal forms and index theorems, it improves some results of Birkhoff and Guenther [3], there is no analog in the differential case. The second method uses a $q$-analog of Poincaré asymptotics expansions and the non abelian cohomology $H^{1}\left(\mathbf{E}_{q}, \Lambda\right)$ of some sheaves $\Lambda$ on the (loxodromic) elliptic curve $\mathbf{E}_{q}:=\mathbf{C}^{*} / q^{\mathbf{Z}}$, it parallels some results of Malgrange and Sibuya (after Birkhoff, Balser-Jürkat-Lutz) in the differential case. The third method uses $q$-multisummability, it parallels [17] in the differential case.

The new version of the classification exposed here is based upon a "fundamental group" $\pi_{1, q, w, 1}^{(0)}$ that we named the q-wild fundamental group (3), a $q$-analog of the wild fundamental group introduced by the first author in the differential case [7], [17]. There is an equivalence of (tannakian) categories between the category of finite dimensional representations of this $q$-wild fundamental group and the category of $q$-difference modules (with integral slopes), moreover the image of a representation is "the" $q$-difference Galois group of the corresponding module. This classification is in the style of the Riemann-Hilbert correspondence for regular-singular meromorphic linear differential equations and should have similar (important...) applications.

\footnotetext{
${ }^{(1)}$ This is explained in section 2.2. For the definition and properties of slopes, see section 2 and 32 .

${ }^{(2)}$ For the simple groups $S L(n, \mathbf{C}), S O(n, \mathbf{C}), S p(2 n, \mathbf{C})$ there are explicit solutions with generalized $q$-hypergeometric difference equations due to J. Roques, $c f$. section 5.1

${ }^{(3)}$ In $\pi_{1, q, w, 1}^{(0)}$, the subsript 1 is for the analogy with $\pi_{1}, q$ is clear, $w$ is for wild, the last 1 is for integral slopes case (i.e.with denominator 1 ) and the superscript ${ }^{(0)}$ is for local at 0 .
} 
Of course there is a "trivial" candidate for a $q$-wild fundamental group satisfying our requirements: the tannakian Galois group $\operatorname{Gal}\left(\mathcal{E}_{1}^{(0)}\right)$ of the tannakian category $\mathcal{E}_{1}^{(0)}$ of our $q$-modules, but this (proalgebraic) group is "too abstract and too big", our purpose was to get a smaller fundamental group (as small as possible!) which is Zariski dense in the tannakian Galois group and to describe it explicitly. (As a byproduct, we shall get finally a complete description of the tannakian Galois group itself.) It is important to notice that the tannakian Galois group is an algebraic object, but that the construction of the smaller group is based upon transcendental techniques (complex analysis). This is similar to what happens with the Riemann-Hilbert correspondance.

We will see that it is possible to write:

$$
\operatorname{Gal}\left(\mathcal{E}_{1}^{(0)}\right)=\mathfrak{S t} \rtimes \operatorname{Gal}\left(\mathcal{E}_{p, 1}^{(0)}\right)
$$

where (4), by definition, $\operatorname{Gal}\left(\mathcal{E}_{p, 1}^{(0)}\right):=\operatorname{Hom}_{g r}\left(\mathbf{E}_{q}, \mathbf{C}^{*}\right) \times \mathbf{C}$ and $\mathfrak{S t}$ is a prounipotent group (named the Stokes group). We can replace $\operatorname{Gal}\left(\mathcal{E}_{1}^{(0)}\right)$ by an equivalent datum, the action of $\operatorname{Gal}\left(\mathcal{E}_{p, 1}^{(0)}\right)$ on the Lie algebra $\mathfrak{s t}$ of $\mathfrak{S t}$. We denote this datum as a semi-direct product $\mathfrak{s t} \rtimes \operatorname{Gal}\left(\mathcal{E}_{p, 1}^{(0)}\right)$.

We build a free Lie algebra $L$ generated by an infinite family of symbols $\dot{\Delta}_{i}^{(\delta, \bar{c})}\left(\delta \in \mathbf{N}^{*}, \bar{c} \in \mathbf{E}_{q}, i=1, \ldots, \delta\right)$ and $\dot{\Delta}^{(0)}$, the (pointed) $q$-alien derivations, endowed with an action of $\operatorname{Gal}\left(\mathcal{E}_{p, 1}^{(0)}\right)_{s}:=\operatorname{Hom}_{g r}\left(\mathbf{E}_{q}, \mathbf{C}^{*}\right)$, and a natural $\operatorname{Gal}\left(\mathcal{E}_{p, 1}^{(0)}\right)_{s}$-equivariant map $L \rightarrow \tilde{\mathfrak{s t}}:=\mathfrak{s t} \oplus \mathbf{C} \log \dot{\Delta}^{(0)}$. Then, by definition:

$$
\pi_{1, q, w, 1}^{(0)}:=L \rtimes \operatorname{Gal}\left(\mathcal{E}_{p, 1}^{(0)}\right)_{s}
$$

and we prove that the natural map

$$
\operatorname{Rep}\left(\operatorname{Gal}\left(\mathcal{E}_{1}^{(0)}\right)\right) \rightarrow \operatorname{Rep}\left(\pi_{1, q, w, 1}^{(0)}\right)
$$

is an isomorphism.

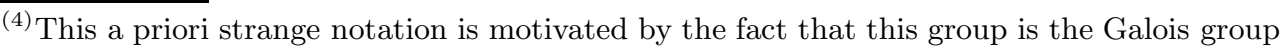
of the category of pure modules.
} 
As a byproduct, we prove that, for some convenient pronilpotent completion $L^{\dagger}$ (introduced in section 3.6 and studied in the appendix) of the free Liealgebra the map:

$$
\exp \left(L^{\dagger}\right) \rtimes G_{p, 1, s}^{(0)} \rightarrow \exp (\tilde{\mathfrak{s t}}) \rtimes G_{p, 1, s}^{(0)}=\mathfrak{S t} \rtimes G_{p, 1}^{(0)}=G_{1}^{(0)}
$$

is an isomorphism of proalgebraic groups. It is an "explicit description" of the tannakian group $G_{1}^{(0)}$.

The construction of $L$ and the proof of its main properties is the outcome of a quite long process (in three steps: [20, [21 and the present article) and uses some deep results of $\mathbf{2 2}$. In 20 we built some (pointed) $q$-alien derivations $\dot{\Delta}_{a}^{\delta}$ belonging to $\mathfrak{s t}^{(5)}$, we interpreted them using $q$-Borel-Ramis transform and we got the "first level" of our construction (the "linear case" as in the two-slopes case). In [21] we proved the Zariski density of the Lie algebra generated by the $q$-alien derivations and we gave a first (awkward...) tentative of devissage in order to "free" a convenient subset of an extended set of alien derivations. Here we finally give "the good" devissage and we prove the freeness theorem (theorem 3.8). The freeness property is absolutely crucial, it allows a very easy computation of the representations of the $q$-wild fundamental group and in particular the solution of the inverse problem.

The ( $q$-Gevrey) devissage used in the present article is based upon the ( $q$-Gevrey) devissage of the non-abelian cohology sets of some sheaves of unipotent groups on $\mathbf{E}_{q}$ and its relations with the $q$-alien derivations. We think that this devissage is interesting by itself and will give later some relations between some $H^{1}\left(\mathbf{E}_{q}, \Lambda\right)$ and some representations of algebraic groups.

The underlying idea of our construction is that the knowledge of a $q$ difference module is equivalent to the knowledge of its formal invariants and of the corresponding $q$-Stokes phenomena (in the sense of [22]). This is similar to what happens in the differential case, but unfortunately there is a major difference, here the entries of the Stokes matrices are $q$-constants, that is elliptic functions on $\mathbf{E}_{q}$, and we would like instead some matrices belonging to $\mathrm{GL}_{n}(\mathbf{C})$ (the $q$-difference Galois groups are defined on $\mathbf{C}$ ). This motivates

\footnotetext{
${ }^{(5)}$ The pointed $q$ alien derivations are $q$-analog of the algebraic pointed alien derivation introduced in 16. The name comes from the fact that in the simplest cases the MartinetRamis pointed alien derivations "coincide" with the derivations introduced before by J. Ecalle under this name. For a proof $c f$. [15.
} 
the replacement of Stokes matrices by $q$-alien derivations (using residues) introduced in [20]: a trick to reduce the field of constants from $\mathcal{M}\left(\mathbf{E}_{q}\right)$ to $\mathbf{C}$.

As a byproduct of our classification theorem we get a $q$-analog of the Ramis density theorem of the differential case [17].

At the end of the story there is a fascinating parallel between the differential and the $q$-difference case. However, it was impossible (in any case for us...) to mimick the differential approach which is essentially based upon the concept of solution, because in the $q$-difference case the solutions behave badly by tensor products. Hence we followed a new path using (roughly speaking) categories in place of solutions.

For more details about the analogies between the $q$-wild fundamental group and the wild fundamental group of the differential case the reader can have a look to the introduction of $[20$ (6).

For each point $\bar{\alpha} \in \mathbf{E}_{q}$, we can consider the semi-direct product of the free Lie algebra generated by the symbols $\dot{\Delta}_{\bar{\alpha}}^{\delta, \bar{\alpha}^{\delta}}\left(\delta \in \mathbf{N}^{*}\right)$ by $\mathbf{C}^{*}$ (the action of $\mathbf{C}^{*}$ corresponding to the grading $\delta$ ). The corresponding category of representations is isomorphic to the category of representations of a quotient of $\pi_{1, q, w, 1}$. Similar groups appear in the linear differential case, in the non linear differential case (Lie algebras of Ecalle pointed alien derivations $(7)$ ) and in the theory of the cosmic Galois group of Connes-Marcolli 5. These groups are in some sense "motivic groups" ( $c f$. also [1] 5. Coda(8) $)$, therefore we can interpret our result as a "motivic version" of the local classification of the $q$-difference modules.

1.1.2. The inverse problem of the Galois theory of q-difference equations. - Using the $q$-wild fundamental group we can imitate the solution of the local inverse problem in the differential case due to the first author. The

\footnotetext{
${ }^{(6)}$ In fact it is possible to get a perfect analogy if one replaces the free resurgent algebra of the wild fundamental group by a bigger free Lie algebra endowed with an action not only of $\mathbf{Z}$ but of its proalgebraic completion $\operatorname{Hom}_{g r}\left(\mathbf{C}^{*}, \mathbf{C}^{*}\right) \times \mathbf{C}$, we will return to this problem in a future paper.

${ }^{(7)}$ The Lie algebra generated by the Ecalle pointed alien derivations $\left\{\dot{\Delta}_{n}\right\}_{n \in \mathbf{N} *}$ is free, the grading corresponding to the rescaling of $e^{-1 / x}$. There is a dictionary between MartinetRamis classification of saddle-nodes and some representations of this algebra [33.

(8) "Ce groupe d'une ubiquité stupéfiante", page 16
} 
problem is to find necessary and sufficient conditions on a complex linear algebraic group in order that this group be the $q$-difference Galois group of a local meromorphic $q$-difference module with integral slopes $\left(q \in \mathbf{C}^{*},|q| \neq 1\right)$.

As in the differential case we get easily some necessary conditions using the algebraic group $V(G):=G / L(G)$ (where $L(G)$ is the invariant subgroup generated by all the maximal tori of $G$ ) and a tannakian argument. In the differential case the corresponding conditions are sufficient, but here it is no longer the case, there appears a new necessary condition involving some type of co-weight on a maximal torus (existence of a $\Theta$-structure(9).). Adding this condition we get a set of necessary and sufficient conditions. It follows in particular that a Borel subgroup of a reductive group is the $q$-difference Galois group of a local meromorphic $q$-difference module with integral slopes.

In [29] and [30] the second author proved a classification theorem for regular singular $q$-difference modules, involving the local modules at 0 et $\infty$ and an invertible elliptic connection matrix (in Birkhoff style) and derived a description of the corresponding Galois group and of a Zariski dense subset of this group. We extend these results to the general case. Using this extension and the solution of the local inverse problem we get a partial solution of the global inverse problem. We prove in particular that every connected reductive group is the $q$-difference Galois group of a rational q-difference module.

1.2. Contents of the paper. - We now briefly sketch the organisation of the paper. General notations and conventions are explained in the next subsection 1.3 ,

Sections 2 to 4 are devoted to the "direct problem" of the description of the Galois group of a $q$-difference module (or system, or equation) with integral slopes. In section 2, we review results from our previous work [20, 21] and adapt them to our present needs. In section 3, we proceed to a complete description of the local Galois group. In section 4, we combine this with previous results from [30] to obtain a description of the global Galois group (when it makes sense); this is less complete that section 3 but nevertheless sufficient for our use in section 7 .

$\overline{{ }^{(9)} c f . \text { the definition } 5.13}$ 
Sections 5 to 7 are devoted to the inverse problem. This is introduced in section 5, as well as an important technical tool, the notion of $\Theta$-structure. In section 6 , the local inverse problem is solved. In section 7 , the global inverse problem is tackled.

1.3. General notations. - Let $q \in \mathbf{C}$ be a complex number with modulus $|q|>1$. We write $\sigma_{q}$ the $q$-dilatation operator, so that, for any map $f$ on an adequate domain in $\mathbf{C}$, one has: $\sigma_{q} f(z)=f(q z)$. Thus, $\sigma_{q}$ defines a ring automorphism in each of the following rings: $\mathbf{C}\{z\}$ (convergent power series), $\mathbf{C}[[z]]$ (formal power series), $\mathcal{O}\left(\mathbf{C}^{*}\right)$ (holomorphic functions over $\mathbf{C}^{*}$ ), $\mathcal{O}\left(\mathbf{C}^{*}, 0\right)$ (germs at 0 of elements of $\mathcal{O}\left(\mathbf{C}^{*}\right)$ ). Likewise, $\sigma_{q}$ defines a field automorphism in each of their fields of fractions: $\mathbf{C}(\{z\})$ (convergent Laurent series), $\mathbf{C}((z))$ (formal Laurent series), $\mathcal{M}\left(\mathbf{C}^{*}\right)$ (meromorphic functions over $\left.\mathbf{C}^{*}\right), \mathcal{M}\left(\mathbf{C}^{*}, 0\right)$ (germs at 0 of elements of $\mathcal{M}\left(\mathbf{C}^{*}\right)$ ). The $\sigma_{q}$-invariants elements of $\mathcal{M}\left(\mathbf{C}^{*}, 0\right)$ actually belong to $\mathcal{M}\left(\mathbf{C}^{*}\right)$ and can be considered as meromorphic functions on the quotient Riemann surface $\mathbf{E}_{q}=\mathbf{C}^{*} / q^{\mathbf{Z}}$. Through the mapping $x \mapsto z=e^{2 \mathrm{i} \pi x}$, the latter is identified to the complex torus $\mathbf{C} /(\mathbf{Z}+\mathbf{Z} \tau)$, where $q=e^{2 \mathrm{i} \pi \tau}$. Accordingly, we shall identify the fields $\mathcal{M}\left(\mathbf{C}^{*}, 0\right)^{\sigma_{q}}, \mathcal{M}\left(\mathbf{C}^{*}\right)^{\sigma_{q}}$ and $\mathcal{M}\left(\mathbf{E}_{q}\right)$. We shall write $a \mapsto \bar{a}$ the canonical projection map $\pi: \mathbf{C}^{*} \rightarrow \mathbf{E}_{q}$ and $[c ; q]=\pi^{-1}(\bar{c})=c q^{\mathbf{Z}}$ (a discrete logarithmic $q$-spiral). Last, we shall have use for the function $\theta \in \mathcal{O}\left(\mathbf{C}^{*}\right)$, a Jacobi Theta function such that $\sigma_{q} \theta=z \theta$ and $\theta$ has simple zeroes along $[-1 ; q]$. One then puts $\theta_{c}(z)=\theta(z / c)$, so that $\theta_{c} \in \mathcal{O}\left(\mathbf{C}^{*}\right)$ satisfies $\sigma_{q} \theta_{c}=(z / c) \theta_{c}$ and $\theta_{c}$ has simple zeroes along $[-c ; q]$.

For any two (pro)algebraic groups $G, H$, the set of morphisms from $G$ to $H$ is written $\operatorname{Hom}_{\text {gralg }}(G, H)$. When we want to consider all morphisms of abstract groups, forgetting the (pro)algebraic structure, we write $\operatorname{Hom}_{g r}(G, H)$.

Acknowledgements. - The final redaction of this work was achieved while the second author was an invited professor for three months at the School of Mathematics and Statistics of "Wuda" (Wuhan University, Wuhan, Hubei, People's Republic of China): he wishes to express his gratitude to Wuda for the excellent working conditions there.

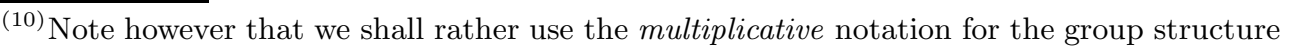
on $\mathbf{E}_{q}$.
} 


\section{Previous results on the structure of the local Galois group}

In this section, we recall the notations and results of [20, 21] and make more precise some of them.

A linear analytic $q$-difference equation at $0 \in \mathbf{C}$ is an equation:

$$
\sigma_{q} X=A X
$$

where $A \in \mathrm{GL}_{n}(\mathbf{C}(\{z\}))$. We shall identify it with the $q$-difference modul ${ }^{(11)}$ :

$$
M_{A}:=\left(\mathbf{C}(\{z\})^{n}, \Phi_{A}\right), \text { where } \Phi_{A}(X):=A^{-1} \sigma_{q} X .
$$

If $B \in \mathrm{GL}_{p}(\mathbf{C}(\{z\}))$, morphisms from $M_{A}$ to $M_{B}$ are described by:

$$
\operatorname{Hom}\left(M_{A}, M_{B}\right)=\left\{F \in \operatorname{Mat}_{p, n}(\mathbf{C}(\{z\})) \mid\left(\sigma_{q} F\right) A=B F\right\} .
$$

The $q$-difference modules over $\mathbf{C}(\{z\})$ form a $\mathbf{C}$-linear neutral tannakian category $\mathcal{E}^{(0)}$, of which we shall now distinguish some particular subcategories. First note that to each $q$-difference module is attached a Newton polygon, which can be described as a sequence $\mu_{1}<\cdots<\mu_{k}$ of rational slopes coming with multiplicities $r_{1}, \ldots, r_{k} \in \mathbf{N}^{*}$. Modules with integral slopes form the full subcategory $\mathcal{E}_{1}^{(0)}$ of $\mathcal{E}^{(0)}$. Modules having only one slope are called pure isoclinic; direct sums of pure isoclinic modules are called pure and they form the full subcategory $\mathcal{E}_{p}^{(0)}$ of $\mathcal{E}^{(0)}$. Pure modules with integral slopes form the full subcategory $\mathcal{E}_{p, 1}^{(0)}$ of both $\mathcal{E}_{p}^{(0)}$ and $\mathcal{E}_{1}^{(0)}$. Pure isoclinic modules of slope 0 are called fuchsian; they form the full subcategory $\mathcal{E}_{f}^{(0)}$ of $\mathcal{E}_{p, 1}^{(0)}$. All these categories are tannakian subcategories of $\mathcal{E}^{(0)}$. Before describing their Galois groups, we shall have a look at their fiber functors.

For any $q$-difference module $M$, holomorphic solutions in $\sigma_{q}$-invariant open subsets of $\left(\mathbf{C}^{*}, 0\right)$ form a sheaf $\mathcal{F}_{M}$ over $\mathbf{E}_{q}$. This sheaf is locally free over the structural sheaf of $\mathbf{E}_{q}$ and thereby defines a holomorphic vector bundle which we also write $\mathcal{F}_{M}$. In case $M$ is given in matricial form $M_{A}=\left(\mathbf{C}(\{z\})^{n}, \Phi_{A}\right)$,

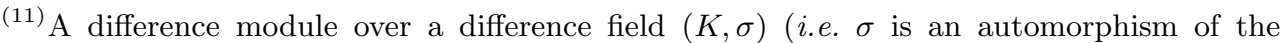
commutative field $K)$ is a pair $M:=(V, \Phi)$, where $V$ is a finite dimensional vector space over $K$ and $\Phi$ a $\sigma$-linear automorphism: $\forall a \in K, \forall x \in V, \Phi(a x)=\sigma(a) \Phi(x)$. Equivalently, $M$ is a finite length left module over the ring $\mathcal{D}_{q, K}:=K\left\langle\sigma, \sigma^{-1}\right\rangle$ of difference operators $\sum a_{i} \sigma^{i}$. Difference modules over $\left(\mathbf{C}(\{z\}), \sigma_{q}\right)$ are called $q$-difference modules.
} 
these sheaf and bundle admit the following descriptions:

$$
\begin{aligned}
\mathcal{F}_{M}(V) & =\left\{X \in \varnothing\left(\pi^{-1}(V), 0\right)^{n} \mid \sigma_{q} X=A X\right\}, \\
\mathcal{F}_{M} & =\frac{\left(\mathbf{C}^{*}, 0\right) \times \mathbf{C}^{n}}{(z, X) \sim(q z, A(z) X)} \longrightarrow \frac{\left(\mathbf{C}^{*}, 0\right)}{z \sim q z}=\mathbf{E}_{q} .
\end{aligned}
$$

In the right hand side of the first (resp. the second) equality, solutions $X \in \varnothing\left(\pi^{-1}(V), 0\right)^{n}$ are taken to be germs at $0 \in \mathbf{C}^{*}$ (resp. the bundle $\left(\mathbf{C}^{*}, 0\right) \times \mathbf{C}^{n}$ to be quotiented is taken to be trivial over the germ of $\mathbf{C}^{*}$ at 0$)$.

The functor $M \leadsto \mathcal{F}_{M}$ is exact, faithful and $\otimes$-compatible and provides a fiber functor on $\mathcal{E}^{(0)}$ over the base $\mathbf{E}_{q}$. Lifting $\mathcal{F}_{M}$ through $\pi$ to an equivariant (trivial) bundle over $\mathbf{C}^{*}$, then taking fibers, we get a family $\left(\omega_{a}^{(0)}\right)_{a \in \mathbf{C}^{*}}$ of fiber functors on $\mathcal{E}^{(0)}$ over $\mathbf{C}$, thus a Galois groupoid with base $\mathbf{C}^{*}$ over the field C. (The reason to consider points in $\mathbf{C}^{*}$ rather than in $\mathbf{E}_{q}$ is that we want to use transcendental constructions. $(12)$.)

On the other hand, $\mathcal{E}^{(0)}$ is endowed with a family $\left(F_{\leq \mu}\right)_{\mu \in \mathbf{Q}}$ of endofunctors such that, for each module $M$, the $F_{\leq \mu} M$ form a filtration of $M$ by subobjects, with jumps at the slopes of $M$. The associated graded module:

$$
\operatorname{gr} M:=\bigoplus \frac{F_{\leq \mu} M}{F_{<\mu} M}
$$

is pure and we get a functor $M \leadsto \operatorname{gr} M$ from $\mathcal{E}^{(0)}$ to $\mathcal{E}_{p}^{(0)}$, which is exact, faithful and $\otimes$-compatible. It is also a retraction of $\mathcal{E}_{p}^{(0)} \subset \mathcal{E}^{(0)}$. This yields a new family of fiber functors on $\mathcal{E}^{(0)}$ :

$$
\hat{\omega}_{a}^{(0)}:=\omega_{a}^{(0)} \circ \mathrm{gr} .
$$

In some sense, $\mathcal{E}_{p}^{(0)}$ is the "formalisation" of $\mathcal{E}^{(0)}$ and we see the $\hat{\omega}_{a}^{(0)}$, resp. the $\omega_{a}^{(0)}$, as points in a formal, resp. an analytic neighborhood of 0 . (The reason for this is that, over the formal category, gr is isomorphic to the identity functor, see [32].)

\footnotetext{
${ }^{(12)}$ It is not feasible in the setting of $q$-difference equations to define a fiber functor as the space of solutions in some big field $K$. Indeed, in order to get a fiber functor in this way, one has to take $K$ rather big; then the fiber functor is defined over the field of constants of $K$, which will be bigger than $\mathbf{C}$. For instance, the natural choice $K=\mathcal{M}\left(\mathbf{C}^{*}\right)$ yields the a Galois group over $\mathcal{M}\left(\mathbf{E}_{q}\right)$.
} 
Whatever the fiber functor used to define it, the Galois group $\operatorname{Gal}\left(\mathcal{E}^{(0)}\right)$ is the semi-direct product of the "formal" Galois group $\operatorname{Gal}\left(\mathcal{E}_{p}^{(0)}\right)$ by a prounipotent group, the kernel of the morphism $\operatorname{Gal}\left(\mathcal{E}^{(0)}\right) \rightarrow \operatorname{Gal}\left(\mathcal{E}_{p}^{(0)}\right)$ dual to gr. (This follows from the existence of the filtration.) Restricting to $\mathcal{E}_{1}^{(0)}$, one gets:

$$
\operatorname{Gal}\left(\mathcal{E}_{1}^{(0)}\right)=\mathfrak{S t} \rtimes \operatorname{Gal}\left(\mathcal{E}_{p, 1}^{(0)}\right),
$$

where $\mathfrak{S t}$ is a prounipotent group.

The goal of this series of papers is the description of the Stokes group $\mathfrak{S t}$ and the Stokes Lie algebrd $(13) \mathfrak{s t}:=\operatorname{Lie}(\mathfrak{S t})$ and its application to the inverse problem in $q$-difference Galois theory. The main tool on the side of $q$-difference equations is theorem 3.11, which describes all Galois groups of systems with integral slopes in terms of representations of a wild fundamental group, actually, the semi-direct product of an infinite dimensional Lie algebra with a proalgebraic group, the tannakian formal Galois group of the category of systems with integral slopes. We obtain it with the help of an explicit family of galoisian Stokes operators built by the authors together with Changgui Zhang in [22] and used there to get an analytic classification of $q$-difference modules. It was proved in previous work $[\mathbf{2 0}, \mathbf{2 1}$ that we thus obtain a generating family. The analytic classification and representations of $\mathfrak{s t}$ are, in some sense, two models of the same thing, which allows us to give a precise description of the latter. In this comparison, the filtration above plays a crucial role and we shall now have a closer look at it.

Convention. - As already said, any object of $\mathcal{E}^{(0)}$ is equivalent to some $M_{A}$. It can moreover be shown that one may always choose $A$ in so-called BirkhoffGuenther normal form; in our case of interest, this is explained at the beginning of 2.2. This implies that $A \in \mathrm{GL}_{n}\left(\mathbf{C}\left[z, z^{-1}\right]\right) \subset \mathrm{GL}_{n}(\mathbf{C}(\{z\})) \cap \mathrm{GL}_{n}\left(\varnothing\left(\mathbf{C}^{*}\right)\right)$, so that the above definitions are simplified to:

$$
\begin{aligned}
\mathcal{F}_{M}(V) & =\left\{X \in \varnothing\left(\pi^{-1}(V)\right)^{n} \mid \sigma_{q} X=A X\right\}, \\
\mathcal{F}_{M} & =\frac{\mathbf{C}^{*} \times \mathbf{C}^{n}}{(z, X) \sim(q z, A(z) X)} \longrightarrow \frac{\mathbf{C}^{*}}{z \sim q z}=\mathbf{E}_{q} .
\end{aligned}
$$

Moreover, starting from a module $M_{A}=\left(\mathbf{C}(\{z\})^{n}, \Phi_{A}\right)$ such that $A \in$ $\mathrm{GL}_{n}\left(\mathbf{C}\left[z, z^{-1}\right]\right)$, a module $M_{B}=\left(\mathbf{C}(\{z\})^{p}, \Phi_{B}\right)$ such that $B \in \mathrm{GL}_{p}\left(\mathbf{C}\left[z, z^{-1}\right]\right)$,

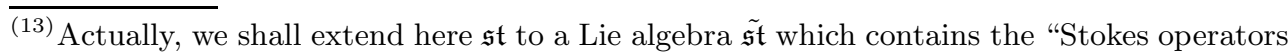
of level 0", that is the unipotent part of the fuchsian Galois group, corresponding to the $q$-logarithm. 
and a morphism $F: M_{A} \rightarrow M_{B}, F \in \operatorname{Mat}_{p, n}(\mathbf{C}(\{z\}))$, it follows from the relation $\left(\sigma_{q} F\right) A=B F \Rightarrow \sigma_{q} F=B F A^{-1}$ that $F$ is holomorphic over $\mathbf{C}^{*}$ (the functional equation allows one to expand by a factor $|q|>1$ any punctured disk of convergence). Thus, in order to have a more concrete description of the fiber functors $\omega_{a}^{(0)}$ and $\hat{\omega}_{a}^{(0)}$, we shall now restrict to the essential full tannakian subcategory of $\mathcal{E}^{(0)}$ made of $q$-difference modules $M_{A}$ such that $A \in \mathrm{GL}_{n}\left(\mathbf{C}\left[z, z^{-1}\right]\right)$. We shall keep the notation $\mathcal{E}^{(0)}$ for this smaller (but equivalent) category. Then, one has canonical identifications $\omega_{a}^{(0)}\left(M_{A}\right)=\mathbf{C}^{n}$, $\omega_{a}^{(0)}\left(M_{B}\right)=\mathbf{C}^{p}$ and $\omega_{a}^{(0)}(F)=F(a)$. A similar description of $\hat{\omega}_{a}^{(0)}$ will be given in 2.2 .

2.1. Some facts about tannakian filtrations. - We shall axiomatize the situation described above in the following way. The tannakian constructions given here essentially follow from [28, chap. IV, §2].

We consider a $\mathbf{C}$-linear tannakian category $\mathcal{C}$ endowed with a family of endofunctors $F_{\leq \mu}, \mu \in \mathbf{Q}$ subject to the following constraints:

1. For each object $M$ of $\mathcal{C}$, the family $F_{\leq \mu} M$ is an ascending filtration of $M$ by subobjects. This filtration is separated, exhaustive and it has a finite number of jumps.

2. Defining $\operatorname{gr}^{(\mu)} M:=\left(F_{\leq \mu} M\right) /\left(F_{<\mu} M\right)$ and $\operatorname{gr} M:=\bigoplus_{\mu \in \mathbf{Q}} \operatorname{gr}^{(\mu)} M$, we obtain an endofunctor gr which is exact, faithful and $\otimes$-compatible.

3. The essential image of gr is a neutral tannakian subcategory $\mathcal{C}_{p}$ (we call its objects pure) and the restriction of gr to $\mathcal{C}_{p}$ is the identity functor.

Choosing a fiber functor $\omega_{p}$ from $\mathcal{C}_{p}$ to $\operatorname{Vect}_{\mathbf{C}}^{f}$, we can extend it to a fiber functor $\omega:=\omega_{p} \circ$ gr on $\mathcal{C}$, thus making the latter a neutral tannakian category. We call $G, G_{p}$ the corresponding Galois groups, seen as proalgebraic groups over $\mathbf{C}$ : these are respectively the groups of $\mathbf{C}$-valued points of the affine group schemes $\underline{\mathrm{Aut}}^{\otimes}(\omega)$ and $\underline{\mathrm{Aut}}^{\otimes}\left(\omega_{p}\right)$.

By tannakian duality, the inclusion functor $i$ from $\mathcal{C}_{p}$ into $\mathcal{C}$ and its retraction gr induce morphisms of proalgebraic groups gr* $: G_{p} \rightarrow G$ and its section $i^{*}: G \rightarrow G_{p}$. Calling $S$ the kernel of $i^{*}$, we get an exact sequence of proalgebraic groups:

$$
1 \rightarrow S \rightarrow G \rightarrow G_{p} \rightarrow 1 .
$$

Since $i^{*} \circ \operatorname{gr}^{*}=(\operatorname{gr} \circ i)^{*}=\operatorname{Id}_{G_{p}}$, this is actually a semi-direct product:

$$
G=S \rtimes G_{p} .
$$


Example 2.1. - The above description applies to $\mathcal{E}^{(0)}$ and $\mathcal{E}_{p}^{(0)}$ as explained in the previous subsection, and so will do all constructions in the present subsection. However, in this paper, from 2.2 on, we shall rather take $\mathcal{E}_{1}^{(0)}$ and $\mathcal{E}_{p, 1}^{(0)}$. Hence, the filtrations on subobjects will have jumps at the integers only, and the functor gr will be $\mathbf{Z}$-graded rather than $\mathbf{Q}$-graded. If we restrict to $\mu \in \mathbf{Z}$, we shall therefore have $F_{<\mu} M=F_{\leq \mu-1} M$. The corresponding Galois groups will be $G:=\operatorname{Gal}\left(\mathcal{E}_{1}^{(0)}\right)$ and $G_{p}:=\operatorname{Gal}\left(\mathcal{E}_{p, 1}^{(0)}\right)$ and the factor $S$ will be denoted $\mathfrak{S t}$ and called the Stokes group.

We shall have use for the following description of the proalgebraic structures on $G, G_{p}$ and $S$. We start with $G$. For each object $M$ of $\mathcal{C}$, write $<M>$ the full subcategory whose objects are subquotients of direct sums of tensors $M^{\otimes n} \otimes\left(M^{\vee}\right)^{\otimes p}$ : this is a tannakian subcategory and there is a restriction morphism from $G$ to its Galois group $G(M)$, which is a linear algebraic group over C. Actually, the map $\gamma \mapsto \gamma(M)$ is an algebraic morphism of $G$ into $\mathrm{GL}(\omega(M))$ and its image is closed; and the similar map from $G(M)$ to $\mathrm{GL}(\omega(M))$ has the same image and is an isomorphism, whence a canonical identification of $G(M)$ with an algebraic subgroup of $\mathrm{GL}(\omega(M))$. Say that $M^{\prime} \preceq M$ if $M^{\prime}$ is an object of $\langle M\rangle$. Then there is a surjective morphism of algebraic groups from $G(M)$ to $G\left(M^{\prime}\right)$. In this way, the groups $G(M)$ form a filtered inverse system and the surjective maps $G \rightarrow G(M)$ make $G$ the inverse limit of the $G(M)$. Morphisms from $G$ to an arbitrary algebraic group $G^{\prime}$ are defined through the equality:

$$
\operatorname{Hom}\left(G, G^{\prime}\right):=\lim _{\rightarrow} \operatorname{Hom}_{\text {gralg }}\left(G(M), G^{\prime}\right) .
$$

(The notation Hom $_{\text {gralg }}$ was defined in 1.3.) Note that the subobjects $F_{\leq \mu} M$, the subquotients $\operatorname{gr}^{(\mu)} M$ and therefore gr $M$ itself all are objects of $\langle M\rangle$, whence a surjective map of algebraic groups $G(M) \rightarrow G(\operatorname{gr} M)$. But, from the equality $\omega_{p} \circ \mathrm{gr}=\omega$ follows a canonical identification of $G(\operatorname{gr} M)$ with $G_{p}(M)$, whence a surjective map $G(M) \rightarrow G_{p}(M)$. Calling $S(M)$ the kernel of this map (an algebraic group), one has first a semidirect decomposition $G(M)=S(M) \rtimes G_{p}(M)$, second an inverse system of exact sequences

$$
1 \rightarrow S(M) \rightarrow G(M) \rightarrow G_{p}(M) \rightarrow 1
$$

whose inverse limit is the sequence seen before.

For any $M$ in $\mathcal{C}$, the vector space $V:=\omega(M)=\omega(\operatorname{gr} M)=\omega_{p}(\operatorname{gr} M)$ admits: 
- a separated exhaustive descending filtration by the $V_{\leq \mu}:=\omega\left(F_{\leq \mu} M\right)$, $\mu \in \mathbf{Q}$, with a finite number of jumps;

- a graduation by the $V^{(\mu)}:=\omega\left(\operatorname{gr}^{(\mu)} M\right), \mu \in \mathbf{Q}$, with a finite number of non trivial components.

Moreover, the filtration is the one associated with the graduation:

$$
V_{\leq \mu}=\bigoplus_{\nu \leq \mu} V^{(\nu)}
$$

In the terminology of [28, it is scindée (split), thus admissible. These structures are determined by $M_{0}:=\operatorname{gr} M$. Using them, one can define the following linear algebraic groups:

1. The group of automorphisms of $V$ respecting the filtration:

$$
\mathrm{GL}_{M_{0}}(V):=\left\{f \in \mathrm{GL}(V) \mid \forall \mu \in \mathbf{Q}, f\left(V_{\leq \mu}\right) \subset V_{\leq \mu}\right\} .
$$

2. The group of automorphisms of $V$ respecting the graduation:

$$
\overline{\mathrm{GL}}_{M_{0}}(V):=\left\{f \in \mathrm{GL}(V) \mid \forall \mu \in \mathbf{Q}, f\left(V^{(\mu)}\right) \subset V^{(\mu)}\right\}=\bigoplus_{\mu \in \mathbf{Q}} \mathrm{GL}\left(V^{(\mu)}\right) .
$$

3. The group of automorphisms of $V$ respecting the filtration and inducing the identity on the graduation:

$$
\mathfrak{G}_{M_{0}}(V):=\left\{f \in \operatorname{GL}(V) \mid \forall \mu \in \mathbf{Q},\left(f-\operatorname{Id}_{V}\right)\left(V_{\leq \mu}\right) \subset V_{<\mu}\right\} .
$$

Roughly, these three groups respectively correspond (in matricial form) to block upper triangular matrices, to block diagonal matrices and to unipotent block upper triangular matrices. The latter two groups are subgroups of the first one with trivial intersection.

There is a natural surjective morphism of algebraic groups from $\mathrm{GL}_{M_{0}}(V)$ onto $\overline{\mathrm{GL}}_{M_{0}}(V)$. Its kernel is $\mathfrak{G}_{M_{0}}(V)$, whence an exact sequence:

$$
1 \rightarrow \mathfrak{G}_{M_{0}}(V) \rightarrow \mathrm{GL}_{M_{0}}(V) \rightarrow \overline{\mathrm{GL}}_{M_{0}}(V) \rightarrow 1 .
$$

Actually, the surjective morphism admits an obvious section, whence a semidirect decomposition:

$$
\mathrm{GL}_{M_{0}}(V)=\mathfrak{G}_{M_{0}}(V) \rtimes \overline{\mathrm{GL}}_{M_{0}}(V) .
$$

The group $\mathfrak{G}_{M_{0}}(V)$ is unipotent (more on this further below). Its (nilpotent) Lie algebra can be characterized as the set of endomorphisms on $V$ respecting the filtration and trivial (null) on the graduation:

$$
\mathfrak{g}_{M_{0}}(V):=\left\{f \in \operatorname{gl}(V) \mid \forall \mu \in \mathbf{Q}, f\left(V_{\leq \mu}\right) \subset V_{<\mu}\right\} .
$$


Now any "galoisian" automorphism $\gamma \in G(M) \subset \mathrm{GL}(\omega(M))$ stabilises images of subobjects, in particular the $\omega\left(F_{\leq_{\mu}} M\right)$ and the $\omega\left(F_{<\mu} M\right)$; and descends to subquotients, in particular the $\omega\left(\operatorname{gr}^{(\mu)}(M)\right.$. With the notations just introduced, we thus have an embedding of exact sequences:

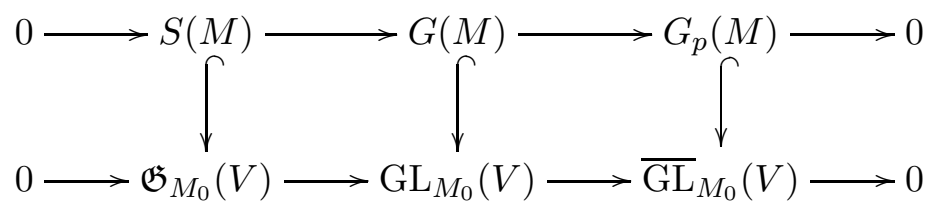

Note in particular that, since $S(M)$ embeds into $\mathfrak{G}_{M_{0}}(V)$, it is a unipotent group and its Lie algebra $s(M)$ is nilpotent. We have:

$$
\begin{aligned}
S(M) & =\exp s(M)=1+s(M), \\
\mathfrak{G}_{M_{0}}(V) & =\exp \mathfrak{g}_{M_{0}}(V)=1+\mathfrak{g}_{M_{0}}(V) .
\end{aligned}
$$

We put, for all $\delta \geq 0$ :

$$
\mathfrak{g}_{M_{0}}^{>\delta}(V):=\left\{f \in \operatorname{gl}(V) \mid \forall \mu \in \mathbf{Q}, f\left(V_{\leq \mu}\right) \subset V_{<\mu-\delta}\right\} .
$$

(In matricial form, this corresponds to upper triangular nilpotent matrices with decreasing orders of nilpotency.) This gives a descending separated exhaustive filtration of $\mathfrak{g}_{M_{0}}(V)$ by ideals, with a finite number of jumps, and we have:

$$
\mathfrak{g}_{M_{0}}^{>0}(V)=\mathfrak{g}_{M_{0}}(V) \text { and }\left[\mathfrak{g}_{M_{0}}^{>\delta}(V), \mathfrak{g}_{M_{0}}^{>\delta^{\prime}}(V)\right] \subset \mathfrak{g}_{M_{0}}^{>\delta+\delta^{\prime}}(V) .
$$

We are interested in the induced filtration on the Stokes Lie algebras:

$$
s^{>\delta}(M):=s(M) \cap \mathfrak{g}_{M_{0}}^{>\delta}(V) .
$$

This gives again a descending separated exhaustive filtration of $s(M)$ by ideals, with a finite number of jumps, such that:

$$
s^{>0}(M)=s(M) \text { and }\left[s^{>\delta}(M), s^{>\delta^{\prime}}(M)\right] \subset s^{>\delta+\delta^{\prime}}(M) .
$$

Last, we introduce the group counterparts:

$$
\begin{aligned}
& \mathfrak{G}_{M_{0}}^{>\delta}(V):=1+\mathfrak{g}_{M_{0}}^{>\delta}(V), \\
& S^{>\delta}(M):=1+s^{>\delta}(M)=S(M) \cap \mathfrak{G}_{M_{0}}^{>\delta}(V) .
\end{aligned}
$$

This yields two descending separated exhaustive filtrations by normal subgroups, with a finite number of jumps, such that:

$$
\begin{array}{llrl}
\mathfrak{G}_{M_{0}}^{>0}(V)=\mathfrak{G}_{M_{0}}(V) & \text { and }\left[\mathfrak{G}_{M_{0}}^{>\delta}(V), \mathfrak{G}_{M_{0}}^{>\delta^{\prime}}(V)\right] & \subset \mathfrak{G}_{M_{0}}^{>\delta+\delta^{\prime}}(V) \\
S^{>0}(M)=S(M) & \text { and }\left[S^{>\delta}(M), S^{>\delta^{\prime}}(M)\right] & \subset S^{>\delta+\delta^{\prime}}(M) .
\end{array}
$$


Now we return to our global Galois groups. Since the exact sequence $1 \rightarrow S \rightarrow G \rightarrow G_{p} \rightarrow 1$ is the inverse limit of the exact sequences $1 \rightarrow$ $S(M) \rightarrow G(M) \rightarrow G_{p}(M) \rightarrow 1$, we see that $S$ is a prounipotent group, equipped with a separated exhaustive filtration by normal subgroups $S^{>\delta}$. Its Lie algebra (see [6, A.7]) $s=\operatorname{Lie} S:=\lim s(M)$ is likewise equipped with a separated exhaustive filtration by ideals $s^{\leftarrow \delta}$, and it is a pronilpotent Lie algebra. Actually, $s$ inherits the inverse limit topology of the discrete topologies on the $s(M)$, which has as a fundamental system of neighborhoods of 0 this filtration; and $s$ is Hausdorff and complete for that topology.

2.2. Overall structure and representations of $\operatorname{Gal}\left(\mathcal{E}_{1}^{(0)}\right)$. - We now make an important assumption:

\section{From now on, we shall restrict to modules with integral slopes.}

The reason is that we then have explicit normal forms, and we are going to use them heavily $\stackrel{(14)}{ }$. Indeed, any pure module $M_{0}$ with integral slopes $\mu_{1}<\cdots<\mu_{k}$ and multiplicities $r_{1}, \ldots, r_{k}$ can be described as $M_{A_{0}}:=$ $\left(\mathbf{C}(\{z\})^{n}, \Phi_{A_{0}}\right)$, and any module $M$ such that $\operatorname{gr} M \approx M_{0}$ can be described as $M_{A}:=\left(\mathbf{C}(\{z\})^{n}, \Phi_{A}\right)$ (see equation (2)), with:

$(5)$

$$
A_{0}:=\left(\begin{array}{ccccc}
z^{\mu_{1}} A_{1} & \ldots & \ldots & \ldots & \ldots \\
\ldots & \ldots & \ldots & 0 & \ldots \\
0 & \ldots & \ldots & \ldots & \ldots \\
\ldots & 0 & \ldots & \ldots & \ldots \\
0 & \ldots & 0 & \ldots & z^{\mu_{k}} A_{k}
\end{array}\right) \text { and } A:=\left(\begin{array}{ccccc}
z^{\mu_{1}} A_{1} & \ldots & \ldots & \ldots & \ldots \\
\ldots & \ldots & \ldots & U_{i, j} & \ldots \\
0 & \ldots & \ldots & \ldots & \ldots \\
\ldots & 0 & \ldots & \ldots & \ldots \\
0 & \ldots & 0 & \ldots & z^{\mu_{k}} A_{k}
\end{array}\right) \text {, }
$$

where, for $1 \leq i \leq k, A_{i} \in \mathrm{GL}_{r_{i}}(\mathbf{C})$ and where, for $1 \leq i<j \leq k$, $U_{i, j} \in \operatorname{Mat}_{r_{i}, r_{j}}(\mathbf{C}(\{z\}))$; moreover, one can assume that the coefficients of each block $U_{i, j}$ belong to $\sum_{\mu_{i} \leq \ell<\mu_{j}} \mathbf{C} z^{\ell}$ (Birkhoff-Guenther normal form). Modules $M_{A}:=\left(\mathbf{C}(\{z\})^{n}, \Phi_{A}\right)$ form an essential tannakian subcategory of $\mathcal{E}_{1}^{(0)}$, so that we can restrict all our definitions and constructions to such objects.

\footnotetext{
${ }^{(14)}$ In the general case of rational slopes, van der Put and Reversat obtained a precise description of pure modules and of the Galois group of $\mathcal{E}_{p}^{(0)}$, see 35 . Relying on these results, Virginie Bugeaud has started to extend the methods of the present series of papers to the case of two arbitrary slopes.
} 
The fiber functors $\hat{\omega}_{a}^{(0)}$ and $\omega_{a}^{(0)}$ admit the following concrete description. Let $A, A_{0}$ be as in (5D) and write for short $M:=M_{A}, M_{0}:=M_{A_{0}}$, so that $M_{0}=\operatorname{gr} M$. Then $\hat{\omega}_{a}^{(0)}(M)=\omega_{a}^{(0)}(M)=\omega_{a}^{(0)}\left(M_{0}\right)=\mathbf{C}^{n}$. Now define similarly $B \in \mathrm{GL}_{p}(\mathbf{C}(\{z\}))$ in Birkhoff-Guenther normal form with slopes $\nu_{1}<\cdots<\nu_{l}$ having multiplicities $s_{1}, \ldots, s_{l}$ and $B_{0}$ its graded (block diagonal) component and put $N:=M_{B}, N_{0}:=M_{B_{0}}$, so that $N_{0}=\operatorname{gr} N$. Then any morphism $M \rightarrow N$ is a matrix $F \in \operatorname{Mat}_{p, n}(\mathbf{C}(\{z\}))$ such that $\sigma_{q} F=B F A^{-1}$, so that one easily shows that $F \in \operatorname{Mat}_{p, n}\left(\varnothing\left(\mathbf{C}^{*}\right)\right)$. The corresponding graded morphism $F_{0}:=\operatorname{gr}(F) \in \operatorname{Mat}_{p, n}(\mathbf{C}(\{z\})) \cap \operatorname{Mat}_{p, n}\left(\varnothing\left(\mathbf{C}^{*}\right)\right)$ has $k l$ blocks of sizes $r_{i} \times s_{j}$, those such that $\mu_{i}=\nu_{j}$ coming from $F$, all the other ones being trivial. Then one has:

$$
\begin{aligned}
& \omega_{a}^{(0)}(F)=F(a), \\
& \hat{\omega}_{a}^{(0)}(F)=F_{0}(a) .
\end{aligned}
$$

The Galois groups of $\mathcal{E}_{f}^{(0)}$ and $\mathcal{E}_{p, 1}^{(0)}$ are abelian, so that we can use any fiber functor to describe them. Using the subscript "f" for "fuchsian" and the subscript "p" for "pure", we have:

$$
\begin{aligned}
G_{f}^{(0)} & :=\operatorname{Gal}\left(\mathcal{E}_{f}^{(0)}\right)=\operatorname{Hom}_{g r}\left(\mathbf{C}^{*} / q^{\mathbf{Z}}, \mathbf{C}^{*}\right) \times \mathbf{C}, \\
G_{p, 1}^{(0)} & :=\operatorname{Gal}\left(\mathcal{E}_{p, 1}^{(0)}\right)=\mathbf{C}^{*} \times G_{f}^{(0)} .
\end{aligned}
$$

(The notation $\operatorname{Hom}_{g r}$ was defined in 1.3.) We also write $G_{f, s}^{(0)}=\operatorname{Hom}_{g r}\left(\mathbf{C}^{*} / q^{\mathbf{Z}}, \mathbf{C}^{*}\right.$ ) the semi-simple component of the fuchsian group $G_{f}^{(0)}$; its elements are identified with (abstract group) morphisms $\mathbf{C}^{*} \rightarrow \mathbf{C}^{*}$ that send $q$ to 1 . Likewise, we write $G_{f, u}^{(0)}=\mathbf{C}$ the unipotent component of $G_{f}^{(0)}$ and $T_{1}^{(0)}=\mathbf{C}^{*}$ the "theta torus" component of $G_{p, 1}^{(0)}$; the latter should be compared with the "exponential torus" component of the wild fundamental group of differential equations.

\footnotetext{
$\overline{{ }^{(15)} \text { For details }}$ on this analogy, see the introduction of $[\mathbf{2 0}]$ and the conclusion of [21].
} 
Taking again $A$ in form (5), the representation of $G_{p, 1}^{(0)}=G_{f, s}^{(0)} \times G_{f, u}^{(0)} \times T_{1}^{(0)}$ corresponding to $M:=M_{A}$ by tannakian duality is the following:

$$
(\gamma, \lambda, t) \mapsto\left(\begin{array}{ccccc}
t^{\mu_{1}} \gamma\left(A_{1, s}\right) A_{1, u}^{\lambda} & \ldots & \ldots & \ldots & \ldots \\
\ldots & \ldots & \ldots & 0 & \ldots \\
0 & \ldots & \ldots & \ldots & \ldots \\
\ldots & 0 & \ldots & \ldots & \ldots \\
0 & \ldots & 0 & \ldots & t^{\mu_{k}} \gamma\left(A_{k, s}\right) A_{k, u}^{\lambda}
\end{array}\right)
$$

We wrote $A_{i}=A_{i, s} A_{i, u}$ the Jordan decomposition into semi-simple and unipotent component, and $\gamma\left(A_{i, s}\right)$ means $\gamma$ operating on eigenvalues of $A_{i}$.

As explained in example 2.1, we now write $\mathfrak{S t}$ the kernel of $i^{*}: G_{1}^{(0)} \rightarrow G_{p, 1}^{(0)}$, a prounipotent proalgebraic group, whence the semidirect decomposition of (41):

$$
G_{1}^{(0)}=\mathfrak{S t} \rtimes G_{p, 1}^{(0)} .
$$

We write $\mathfrak{s t}$ the Lie algebra of $\mathfrak{S t}$; it is pronilpotent, see the end of 2.1 ,

2.3. First look at the structure of $\mathfrak{S t}$ and $\mathfrak{s t}$. - Let us characterize Stokes operators, i.e. elements of the Stokes group $\mathfrak{S t}$ and alien derivations, i.e. elements of the Stokes Lie algebra $\mathfrak{s t}$. Let $s \in \mathfrak{S t}$, resp. $D \in \mathfrak{s t}$. Their respective images by the representation associated to matrix $A$ (meaning: to module $M_{A}$ ) are

$$
\begin{gathered}
s(A) \in \mathfrak{S t}(A) \subset \mathfrak{G}_{A_{0}}(\mathbf{C}) \subset \mathrm{GL}_{n}(\mathbf{C}), \text { where } \operatorname{Stt}(A):=\mathfrak{S t}\left(M_{A}\right), \\
D(A) \in \mathfrak{s t}(A) \subset \mathfrak{g}_{A_{0}}(\mathbf{C}) \subset \operatorname{gl}_{n}(\mathbf{C}), \text { where } \mathfrak{s t}(A):=\mathfrak{s t}\left(M_{A}\right),
\end{gathered}
$$

where we introduce the following unipotent algebraic group $\mathfrak{G}_{A_{0}}$ and its Lie algebra $\mathfrak{g}_{A_{0}}$ :

$$
\begin{aligned}
\mathfrak{G}_{A_{0}}: & =\left\{\left(\begin{array}{ccccc}
I_{r_{1}} & \ldots & \ldots & \ldots & \ldots \\
\ldots & \ldots & \ldots & \star & \ldots \\
0 & \ldots & \ldots & \ldots & \ldots \\
\ldots & 0 & \ldots & \ldots & \ldots \\
0 & \ldots & 0 & \ldots & I_{r_{k}}
\end{array}\right)\right\} \subset \mathrm{GL}_{n} \\
\mathfrak{g}_{A_{0}} & :=\left\{\left(\left(\begin{array}{ccccc}
0_{r_{1}} & \ldots & \ldots & \ldots & \ldots \\
\ldots & \ldots & \ldots & \star & \ldots \\
0 & \ldots & \ldots & \ldots & \ldots \\
\ldots & 0 & \ldots & \ldots & \ldots \\
0 & \ldots & 0 & \ldots & 0_{r_{k}}
\end{array}\right)\right\} \subset \mathrm{gl}_{n}=\mathrm{Mat}_{n} .\right.
\end{aligned}
$$


Here $I_{r}$ and $0_{r}$ respectively denote the identity and the null matrix of size $r \times r$. The rectangular block $\star$ indexed by $(i, j)$ such that $1 \leq i<j \leq k$ has size $r_{i} \times r_{j}$ and links the diagonal square blocks corresponding to slopes $\mu_{i}$ and $\mu_{j}$.

Globally, $s$ and $D$ are characterized as follows. They must be functorial: if $\left(\sigma_{q} F\right) A=B F$, then

$$
s(B) F_{0}(a)=F_{0}(a) s(A) \text { and } D(B) F_{0}(a)=F_{0}(a) D(A)
$$

(for the chosen base point $a \in \mathbf{C}^{*}$ ). They must be $\otimes$-compatible:

$$
s(A \otimes B)=s(A) \otimes s(B) \text { and } D(A \otimes B)=D(A) \otimes I_{p}+I_{n} \otimes D(B) .
$$

Last, they must be trivial on pure modules:

$$
s\left(A_{0}\right)=I_{n} \text { and } D\left(A_{0}\right)=0_{n} .
$$

The character group of the semi-simple component of $G_{p, 1}^{(0)}$ is:

$$
X\left(G_{f, s}^{(0)} \times T_{1}^{(0)}\right)=\mathbf{E}_{q} \times \mathbf{Z} .
$$

To describe the adjoint action of this group on $\mathfrak{s t}$ therefore amounts to give the decomposition in eigenspaces; note that for the projective limit $\mathfrak{s t}$, we have to complete the direct sum:

$$
\mathfrak{s t}=\bigoplus_{\delta \geq 1} \mathfrak{s t}^{(\delta)}, \text { where } \mathfrak{s t}^{(\delta)}=\bigoplus_{\bar{c} \in \mathbf{E}_{q}} \mathfrak{s t}^{(\delta, \bar{c})} .
$$

(Note that only the weights such that $\delta \geq 1$ are required, because of the triangular structure coming from the functorial filtration theorem.) This decomposition is expressed elementwise as a Fourier decomposition:

$$
\forall D \in \mathfrak{s t}, \forall \sigma \in G_{f, s}^{(0)} \times T_{1}^{(0)}, \sigma D \sigma^{-1}=\sum_{\chi \in X\left(G_{f, s}^{(0)} \times T_{1}^{(0)}\right)}\langle\chi, \sigma\rangle D^{(\chi)},
$$

where, for $\chi=(\delta, \bar{c}) \in \mathbf{Z} \times \mathbf{E}_{q}$ and for $\sigma=(t, \gamma) \in \mathbf{C}^{*} \times \operatorname{Hom}_{g r}\left(\mathbf{C}^{*} / q^{\mathbf{Z}}, \mathbf{C}^{*}\right)$ :

$$
\langle\chi, \sigma\rangle=t^{\delta} \gamma(\bar{c}) \text {. }
$$

Thus, $D=\sum D^{(\delta, \bar{c})}$ (with unicity of the decomposition) and:

$$
\sigma D^{(\delta, \bar{c})} \sigma^{-1}=t^{\delta} \gamma(\bar{c}) D^{(\delta, \bar{c})} .
$$

Since $G_{p, 1}^{(0)}$ is abelian, conjugacy under elements of its unipotent component $G_{f, u}^{(0)}$ fixes all each $\mathfrak{s t}^{(\delta, \bar{c})}$. We shall write $\tau$ the (Zariski-) generator $1 \in \mathbf{C}=$ 
$G_{f, u}^{(0)}$, so that:

$$
\tau \mathfrak{s t}^{(\delta, \bar{c})} \tau^{-1}=\mathfrak{s t}^{(\delta, \bar{c})}
$$

2.4. First look at the representations of $\mathfrak{S t}$ and $\mathfrak{s t}$. - More generally, the semi-simple component of $G_{p, 1}^{(0)}$ operates on $\mathfrak{g}_{A_{0}}$ through $G_{p, 1}^{(0)}(A)=$ $G_{p, 1}^{(0)}\left(A_{0}\right)$, whence a decomposition:

$$
\mathfrak{g}_{A_{0}}=\bigoplus_{\delta \geq 1} \mathfrak{g}_{A_{0}}{ }^{(\delta)}, \text { where } \mathfrak{g}_{A_{0}}{ }^{(\delta)}=\bigoplus_{\bar{c} \in \mathbf{E}_{q}} \mathfrak{g}_{A_{0}}{ }^{(\delta, \bar{c})} .
$$

(And, of course, $\mathfrak{s t}^{(\delta)}(A)=\mathfrak{s t}(A) \cap \mathfrak{g}_{A_{0}}{ }^{(\delta)}$, etc.) More concretely, one can divide matrices in $\mathfrak{g}_{A_{0}}$ in rectangular blocks numbered $(i, j)$ with $1 \leq i<j \leq k$; the block $i, j$ has size $r_{i} \times r_{j}$ and links the (null) square diagonal blocks corresponding to slopes $\mu_{i}$ and $\mu_{j}$. If one assumes moreover that the matrices $A_{i}$ are divided in diagonal blocks corresponding to their eigenvalues, then one can further divide each block $(i, j)$ in rectangular blocks numbered $(d, e) \in \operatorname{Sp} A_{i} \times \operatorname{Sp} A_{j}$. The action of $\sigma=(\gamma, t) \in G_{f, s}^{(0)} \times T_{1}^{(0)}$ (through its image in $\left.\mathfrak{G}_{A_{0}}\right)$ on the block $((i, j),(d, e))$ is multiplication by the nonzero scalar $\frac{t^{\mu_{i}}}{t^{\mu_{j}}} \frac{\gamma(\bar{d})}{\gamma(\bar{e})}$. Thus, the matrices of $\mathfrak{g}_{A_{0}}{ }^{(\delta)}$ are those such that blocks with $\mu_{j}-\mu_{i} \neq \delta$ are all zero and the matrices of $\mathfrak{g}_{A_{0}}{ }^{(\delta, \bar{c})}$ are those matrices of $\mathfrak{g}_{A_{0}}{ }^{(\delta)}$ such that blocks with $d / e \not \equiv c\left(\bmod q^{\mathbf{Z}}\right)$ are all zero. We shall frequently identify $\mathfrak{g}_{A_{0}}{ }^{(\delta)}$, resp. $\mathfrak{g}_{A_{0}}{ }^{(\delta, \bar{c})}$ with the corresponding vector spaces of rectangular matrices, forgetting their null components. For instance, in the case of two slopes $\mu<\nu$ with multiplicities $r, s \in \mathbf{N}^{*}$, the (abelian) Lie algebra $\mathfrak{g}_{A_{0}}$ has a single nontrivial component $\mathfrak{g}_{A_{0}}{ }^{(\delta)}$, with $\delta=\nu-\mu$, and we identify it with $\operatorname{Mat}_{r, s}(\mathbf{C})$.

The conjugacy action of the unipotent component of $G_{p, 1}^{(0)}(A)$ leaves stable each $\mathfrak{g}_{A_{0}}{ }^{(\delta, \bar{c})}$. Recall its Zariski-generator $\tau$ defined at the very end of 2.3 . Writing:

$$
U:=\tau(A)=\tau\left(A_{0}\right)=\left(\begin{array}{ccccc}
A_{1, u} & \ldots & \ldots & \ldots & \ldots \\
\ldots & \ldots & \ldots & 0 & \ldots \\
0 & \ldots & \ldots & \ldots & \ldots \\
\ldots & 0 & \ldots & \ldots & \ldots \\
0 & \ldots & 0 & \ldots & A_{k, u}
\end{array}\right)
$$

we see that:

$$
U \mathfrak{g}_{A_{0}}{ }^{(\delta, \bar{c})} U^{-1}=\mathfrak{g}_{A_{0}}{ }^{(\delta, \bar{c})} .
$$


Now fix $M_{0}, A_{0}$ in $\mathcal{E}_{p, 1}^{(0)}$ as above and call $\rho_{0}$ the attached representation of $G_{p, 1}^{(0)}$. We consider objects $M, A$ in $\mathcal{E}_{1}^{(0)}$ above $M_{0}, A_{0}$ (that is, $\operatorname{gr} M=M_{0}$ ). By tannakian duality, they correspond to representations $\rho$ of $G_{1}^{(0)}=\mathfrak{S t} \rtimes G_{p, 1}^{(0)}$ which restrict to $\rho_{0}$ on $G_{p, 1}^{(0)}$. These representations $\rho$ are in turn in one to one correspondance with representations of $\mathfrak{S t}$ that are compatible with $\rho_{0}$. Translated in terms of representations of $\mathfrak{s t}$, this gives:

Proposition 2.2. - Those representations of $\mathfrak{s t}$ corresponding to objects $M, A$ above $M_{0}, A_{0}$ are exactly those such that:

1. Each $\mathfrak{s t}^{(\delta, \bar{c})}$ is mapped to $\mathfrak{g}_{A_{0}}{ }^{(\delta, \bar{c})}$;

2. The conjugation by $\tau$ in $\mathfrak{s t}$ is intertwined with the conjugation by $U$ in $\mathfrak{g}_{A_{0}}$, i.e. $\rho\left(\tau D \tau^{-1}\right)=U \rho(D) U^{-1}$.

We write $\operatorname{Rep}_{A_{0}}(\mathfrak{s t})$ the set of these representations.

Proof. - Indeed, the first condition expresses compatibility with the semisimple component of the representation $\rho_{0}$.

In this paper, we shall extend the definition of the Stokes Lie algebra to include the fuchsian unipotent component and put:

$$
\tilde{\mathfrak{s t}}:=\mathfrak{s t} \rtimes \operatorname{Lie}\left(G_{f, u}^{(0)}\right)=\mathbf{C} \nu \oplus \mathfrak{s t},
$$

that is, $\tilde{\mathfrak{s t}}$ is generated by $\mathfrak{s t}$ and by $\operatorname{Lie}\left(G_{f, u}^{(0)}\right)=\mathbf{C} \nu$, where $\nu:=\log \tau$. Since $G_{f, u}^{(0)}$ commutes with $G_{f, s}^{(0)} \times T_{1}^{(0)}$, the adjoint action of this group on $\mathbf{C} \nu$ is trivial and we write $\tilde{\mathfrak{s t}}^{(\chi)}:=\mathfrak{s t}^{(\chi)}$ and $\tilde{\mathfrak{s t}}^{(0)}:=\mathbf{C} \nu$.

Corollary 2.3. - Those representations of $\tilde{\mathfrak{s t}}$ corresponding to objects $M, A$ above $M_{0}, A_{0}$ are exactly those such that:

1. Each $\tilde{\mathfrak{s t}}^{(\delta, \bar{c})}$ is mapped to $\mathfrak{g}_{A_{0}}{ }^{(\delta, \bar{c})}$;

2. The element $\nu$ is mapped to $\log U$.

We write $\operatorname{Rep}_{A_{0}}(\tilde{\mathfrak{s t}})$ the set of these representations.

2.5. Explicit generators of $\mathfrak{S t}$. - Let $A_{0}, A$ be as in (5). Then, there is a unique $F \in \mathfrak{G}_{A_{0}}(\mathbf{C}((z)))$ such that $F\left[A_{0}\right]=A$. We write it $\hat{F}_{A}$. The components of the $(i, j)$ block of $\hat{F}_{A}$ have $q$-Gevrey level $\delta:=\mu_{j}-\mu_{i}$, meaning that they are divergent formal series with coefficients $a_{k}$ having a growth of order $q^{k^{2} / 2 \delta}$ (up to some $O\left(R^{k}\right)$ factor). Stokes operators, to be defined herebelow, 
are obtained by "summing" this formal object in various directions then taking quotients of such summations (ambiguities). We consider as candidate "directions of summation" the $q$-spirals $[c ; q]$ in $\mathbf{C}^{*}$, equivalently, the points $\bar{c} \in \mathbf{E}_{q}$. Define:

$$
\Sigma_{A_{0}}:=\left\{\bar{c} \in \mathbf{E}_{q} \mid q^{\mathbf{Z}} c^{\mu_{i}} \operatorname{Sp}\left(A_{i}\right) \cap q^{\mathbf{Z}} c^{\mu_{j}} \operatorname{Sp}\left(A_{j}\right) \neq \emptyset \text { for some } 1 \leq i<j \leq k\right\},
$$

thus a finite subset of $\mathbf{E}_{q}$. Then [31]:

Proposition 2.4. - For all $\bar{c} \in \mathbf{E}_{q} \backslash \Sigma_{A_{0}}$, there is a unique $F \in \mathfrak{G}_{A_{0}}\left(\mathcal{M}\left(\mathbf{C}^{*}\right)\right)$ such that $F\left[A_{0}\right]=A$ and subject to the following constraints: components of the $(i, j)$ block are meromorphic over $\mathbf{C}^{*}$ with at worst poles over $[-c ; q]$, of order $\leq \mu_{j}-\mu_{i}$.

One proves in 22 that, in some adequate sense, this $F$ is asymptotic to $\hat{F}_{A}$. We write it $S_{\bar{c}} \hat{F}_{A}$ and we consider it as a summation of $S_{\bar{c}} \hat{F}_{A}$ in the "direction" $\bar{c} \in \mathbf{E}_{q}$. Thus, elements of $\Sigma_{A_{0}}$ are prohibited directions of summation. The Stokes operators are then defined as:

$$
S_{\bar{c}, \bar{d}} \hat{F}_{A}:=\left(S_{\bar{c}} \hat{F}_{A}\right)^{-1} S_{\bar{d}} \hat{F}_{A} .
$$

These are meromorphic automorphisms of $A_{0}$, and they are galoisian in the following sense: evaluating them at a fixed base point $a \in \mathbf{C}^{*}$ that is not a pole will yield elements of $\mathfrak{S t}(A)$ for the corresponding fiber functor $\hat{\omega}_{a}^{(0)}$. More precisely [21]:

Proposition 2.5. - For all $\bar{c}, \bar{d} \in \mathbf{E}_{q} \backslash \Gamma$ such that $a \notin[-c ; q] \cup[-d ; q]$ (so that $a$ is not a pole):

$$
S_{\bar{c}, \bar{d}} \hat{F}_{A}(a) \in \mathfrak{S t}(A)
$$

and these elements, together with their conjugates under the action of $G_{p, 1}^{(0)}(A)$, are Zariski-generators of $\mathfrak{S t}(A)$.

Since $S_{\bar{c}, \bar{d}} \hat{F}_{A}=\left(S_{\overline{c_{0}}, \bar{c}} \hat{F}_{A}\right)^{-1} S_{\overline{c_{0}}, \bar{d}} \hat{F}_{A}$, we may as well fix $\overline{c_{0}}$ and consider the family of all $S_{\overline{c_{0}}, \bar{c}} \hat{F}_{A}(a)$. The question of their relations thus comes next.

2.6. Explicit generators of $\mathfrak{s t}$. - In order to try to "free" these generators, one goes to the Lie algebra. Fix an arbitrary $\overline{c_{0}} \in \mathbf{E}_{q} \backslash \Sigma_{A_{0}}$. For a given $A$, the map:

$$
\bar{c} \mapsto \log S_{\overline{c_{0}}, \bar{c}} \hat{F}_{A}(a)
$$


is meromorphic on $\mathbf{E}_{q}$ with poles on $\Sigma_{A_{0}}$, with values in $\mathfrak{s t}(A)$. Its residue at $\alpha \in \Sigma_{A_{0}}$ is written:

$$
\Delta_{\alpha}(A):=\operatorname{Res}_{\beta=\alpha} \log S_{\overline{c_{0}}, \beta} \hat{F}_{A}(a) \in \mathfrak{s t}(A) .
$$

Residues at points $\alpha \notin \Sigma_{A_{0}}$ are null, except maybe at the particular point $\bar{a}$, where $a$ encodes the fiber functor; but this one has no intrinsic significance and we shall have no use for it.

Now the above statement may be reinforced as follows. From [20, 21], it follows that the mapping $A \mapsto \Delta_{\alpha}(A)$ is functorial and tensor compatible in the sense of the Stokes Lie algebra (see section 2.3) when defined on all operands; by continuity, this remains true without condition:

Lemma 2.6. - Each mapping $A \leadsto \Delta_{\alpha}(A)$ defines an element $\Delta_{\alpha}$ of $\mathfrak{s t .}$

According to (6) in 2.3, $\Delta_{\alpha}$ admits a decomposition:

$$
\Delta_{\alpha}=\hat{\bigoplus}^{(\delta)}, \quad \Delta_{\alpha}^{(\delta)}=\hat{\bigoplus}^{(\delta, \bar{c})} .
$$

We see the components $\Delta_{\alpha}^{(\delta, \bar{c})}$ as $q$-analogs of alien derivations. It was proved in [21, theorem 3.5] (with slightly different notations) that:

Proposition 2.7. - The $\Delta_{\alpha}$, together with their conjugates under the action of $G_{p, 1}^{(0)}$, are topological generators of $\mathfrak{s t .}$

From the preceding section, we draw:

Theorem 2.8. - The "q-alien derivations" $\Delta_{\alpha}^{(\delta, \bar{c})}$ together with $\nu$ generate topologically the Lie algebra $\tilde{\mathfrak{s t}}$.

Remark 2.9. - It was conjectured at the end of 21 that those " $q$-alien derivations" $\Delta_{\alpha}^{(\delta, \bar{c})}$ such that $\alpha^{\delta}=\bar{c}$ (remember we use a multiplicative notation for the group $\mathbf{E}_{q}$ ), together with their conjugates under the action of $G_{p, 1}^{(0)}$, are topological generators of $\mathfrak{s t}$. This will be proved in section 3.3. Therefore, those $\Delta_{\alpha}^{(\delta, \bar{c})}$ such that $\alpha^{\delta}=\bar{c}$ together with $\nu$ generate topologically the Lie algebra $\tilde{\mathfrak{s t}}$. The condition on $\alpha, \delta, \bar{c}$ can be interpreted in terms of "directions of maximal growth" as in the theory of differential equations.

From considerations related to the classification theory (see section 3.1), one 
can predict that these generators are not free: there should be $\delta$ of them for each pair $\delta, \bar{c}$, but there are $\delta^{2}$. In this respect, the "freeness theorem" of [21 is quite incomplete. We shall here complete it by theorem 3.8 at the end of 3.5 .

2.7. $q$-Gevrey interpolation. - Here, we use [21, §3.3.3]. For each level $\delta \in \mathbf{N} \cup\{\infty\}$, we define a category $\mathcal{E}^{\delta}$ with the same objects as $\mathcal{E}_{1}^{(0)}$ but morphisms having coefficients in the field of $q$-Gevrey series of level $>\delta$ (see definition at the beginning of 2.5). For $\delta=\infty$, the morphisms are analytic and $\mathcal{E}^{\infty}=\mathcal{E}_{1}^{(0)}$. For $\delta=0$, any $\hat{F}_{A}$ is a morphism, so that any $A$ is equivalent to $A_{0}$ and $\mathcal{E}^{0}=\mathcal{E}_{p, 1}^{(0)}$. In between, the interpolating categories $\mathcal{E}^{\delta}$ are related by essentially surjective and (not fully) faithful $\otimes$-compatible inclusion functors $\mathcal{E}^{\delta} \hookrightarrow \mathcal{E}^{\delta-1}$, whence the following diagram:

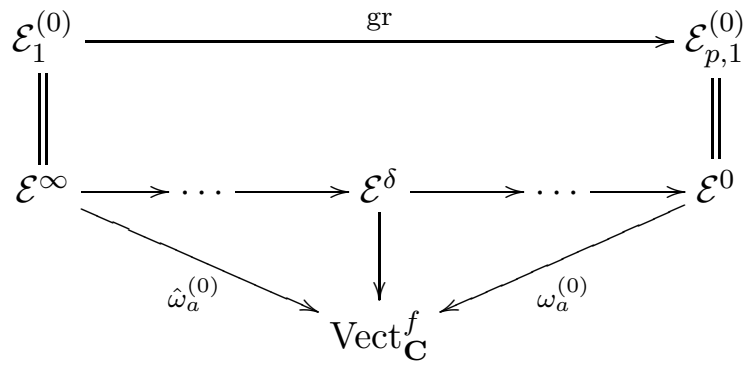

Each $\mathcal{E}^{\delta}$ is tannakian, with the same fiber functors as $\mathcal{E}_{1}^{(0)}$, and its Galois group is a closed subgroup of $G_{1}^{(0)}$ (its elements are $\otimes$-automorphisms of the fiber functor with more constraints imposed by functoriality since there are more morphisms; this is a particular case of [8 prop. 2.21 (b), p. 139]). Actually:

$$
\operatorname{Gal}\left(\mathcal{E}^{\delta}\right)=\mathfrak{S t}^{\leq \delta} \rtimes G_{p, 1}^{(0)},
$$

where $\mathfrak{S t}^{\leq} \leq \delta$ is the subgroup of $\mathfrak{S t}$ with Lie algebra $(16)$ :

$$
\operatorname{Lie}\left(\mathfrak{S t}^{\leq \delta}\right)=\mathfrak{s t}^{\leq \delta}:=\sum_{\delta^{\prime} \leq \delta} \mathfrak{s t}^{\left(\delta^{\prime}\right)}
$$

Thus, $\mathfrak{s t}^{\leq \delta}$ contains in particular all the $\Delta_{\alpha}^{\left(\delta^{\prime}, \bar{c}\right)}$ for $\delta^{\prime} \leq \delta$.

We now define:

$$
\tilde{\mathfrak{s t}}^{\leq \delta}:=\sum_{\delta^{\prime} \leq \delta} \tilde{\mathfrak{s t}}^{\left(\delta^{\prime}\right)}=\mathbf{C} \nu \oplus \mathfrak{s t}^{\leq \delta}
$$

$\overline{{ }^{(16)} \text { This was denoted } \mathfrak{s t}(\delta) \text { in } 21 .}$. 
Then, from what was said before and the grading, one draws:

Proposition 2.10. - The Lie algebra $\tilde{\mathfrak{s t}}^{\leq \delta}$ is generated by $\nu$ and the $\Delta_{\alpha}^{\left(\delta^{\prime}, \bar{c}\right)}$ such that $\alpha^{\delta^{\prime}}=\bar{c}$ for $\delta^{\prime} \leq \delta$.

\section{Structure of the Stokes component}

In this section, we shall describe in detail the structure of $\mathfrak{s t}$ and its representations. We first recall some necessary facts about classification.

3.1. Some useful results on local analytic classification. - These results come from [22, 31. Fix a pure module $M_{0}$ with matrix $A_{0}$ in form (5). The modules formally equivalent to $M_{0}$ are those such that $\operatorname{gr} M \approx M_{0}$. In order to classify them analytically, one rigidifies the situation by introducing "marked pairs" $(M, g)$ made up of an analytic $q$-difference module $M$ and an isomorphism $g: \operatorname{gr}(M) \rightarrow M_{0}$. We then define two such marked pairs $(M, g)$ and $\left(M^{\prime}, g^{\prime}\right)$ to be equivalent if there exists a morphism $f: M \rightarrow M^{\prime}$ such that $g=g^{\prime} \circ \operatorname{gr}(f)$. By standard commutative algebra, such a morphism $f$ is automatically an isomorphism.

The set of equivalence classes of marked pairs is written $\mathcal{F}\left(M_{0}\right)$ and we see it as the space of isoformal analytic classes in the formal class of $M_{0}$. The corresponding classification problem was solved in [22] and we shall use it in 3.3 to get an alternative description of $\operatorname{Rep}_{A_{0}}(\mathfrak{s t})$.

We define the sheaf $\Lambda_{I}\left(M_{0}\right)$ of meromorphic automorphisms of $M_{0}$ infinitely tangent to identity as:

$$
\Lambda_{I}\left(M_{0}\right)(V):=\left\{F \in \mathfrak{G}_{A_{0}}\left(\varnothing\left(\pi^{-1}(V)\right)\right) \mid F\left[A_{0}\right]=A_{0}\right\} .
$$

( $V$ denoting an open subset of $\mathbf{E}_{q}$.) This is a sheaf of unipotent groups over $\mathbf{E}_{q}$, and it is abelian only in the case that $M_{0}$ has one or two slopes; in the former case, it is trivial, in the latter case, it is a vector bundle [31.

Now let $M$ in the formal class of $M_{0}$, with matrix $A$ in form (5). The family of all the $S_{\bar{c}, \bar{d}} \hat{F}_{A}$ for all $\bar{c}, \bar{d} \in \mathbf{E}_{q} \backslash \Sigma_{A_{0}}$ is a cocycle for the above sheaf:

$$
\left(S_{\bar{c}, \bar{d}} \hat{F}_{A}\right)_{\bar{c}, \bar{d}} \in Z^{1}\left(\mathfrak{U}_{A_{0}}, \Lambda_{I}\left(M_{0}\right)\right) .
$$


Here, $\mathfrak{U}_{A_{0}}$ is the covering of $\mathbf{E}_{q}$ by the Zariski open sets $\mathbf{E}_{q} \backslash\{\overline{-c}\}, \bar{c} \in \mathbf{E}_{q} \backslash \Sigma_{A_{0}}$. The conditions on the poles of summations $S_{\bar{c}} \hat{F}_{A}$ imply that each $S_{\bar{c}, \bar{d}} \hat{F}_{A}$ has only poles on $[-c ; q] \cup[-d ; q]$, with multiplicities $\leq \mu_{j}-\mu_{i}$ for the coefficients of the block $(i, j)$. We call privileged such a cocycle and write $Z_{p r}^{1}\left(\mathfrak{U}_{A_{0}}, \Lambda_{I}\left(M_{0}\right)\right)$ the space of privileged cocycles.

Theorem 3.1. - [22, 31] The maps sending A to this cocycle and the latter to its cohomology class induce isomorphisms:

$$
\mathcal{F}\left(M_{0}\right) \rightarrow Z_{p r}^{1}\left(\mathfrak{U}_{A_{0}}, \Lambda_{I}\left(M_{0}\right)\right) \rightarrow H^{1}\left(\mathbf{E}_{q}, \Lambda_{I}\left(M_{0}\right)\right) .
$$

We now describe a $q$-Gevrey interpolation of this classification. Write $\mathfrak{G}_{A_{0}} \geq \delta$ the subgroup of $\mathfrak{G}_{A_{0}}$ defined by the vanishing of all blocks $(i, j)$ such that $0<\mu_{j}-\mu_{i}<\delta$. This is a normal subgroup of $\mathfrak{G}_{A_{0}}$ and each quotient $\mathfrak{G}_{A_{0}} \geq \delta / \mathfrak{G}_{A_{0}} \geq \delta+1$ is abelian, indeed isomorphic to $\mathfrak{g}_{A_{0}}{ }^{(\delta)}$, whence an exact sequence:

$$
0 \rightarrow \mathfrak{g}_{A_{0}}{ }^{(\delta)} \rightarrow \mathfrak{G}_{A_{0}} / \mathfrak{G}_{A_{0}} \geq \delta+1 \rightarrow \mathfrak{G}_{A_{0}} / \mathfrak{G}_{A_{0}} \geq \delta \rightarrow 1 .
$$

This is actually a central extension. It induces a central extension of sheaves:

$$
0 \rightarrow \lambda_{I}^{(\delta)}\left(M_{0}\right) \rightarrow \Lambda_{I}\left(M_{0}\right) / \Lambda_{\bar{I}}^{\geq \delta+1}\left(M_{0}\right) \rightarrow \Lambda_{I}\left(M_{0}\right) / \Lambda_{\bar{I}}^{\geq \delta}\left(M_{0}\right) \rightarrow 1 .
$$

The sheaf $\lambda_{I}\left(M_{0}\right)^{(\delta)}:=\Lambda_{\bar{I}}^{\geq \delta}\left(M_{0}\right) / \Lambda_{\bar{I}}^{\geq \delta+1}\left(M_{0}\right)$ is a sheaf of abelian groups, actually a vector bundle over $\mathbf{E}_{q}$, corresponding by the construction at the beginning of section 2 to a $q$-difference module that is pure isoclinic of slope $\delta$ : it is indeed the direct sum of the equations $\sigma_{q} f=\left(z^{\mu_{i}} A_{i}\right) f\left(z^{\mu_{j}} A_{j}\right)^{-1}$ for $\mu_{j}-\mu_{i}=\delta$. Now, using some non-abelian cohomology from [11], one gets an exact sequence:

$$
0 \rightarrow V^{(\delta)} \rightarrow \mathcal{F}^{\leq \delta}\left(M_{0}\right) \rightarrow \mathcal{F}^{\leq \delta-1}\left(M_{0}\right) \rightarrow 1 .
$$

The meaning of this sequence is the following:

1. The leftmost term $V^{(\delta)}:=H^{1}\left(\mathbf{E}_{q}, \lambda_{I}^{(\delta)}\left(M_{0}\right)\right)$ is a finite dimensional complex vector space (first cohomology of a vector bundle); its dimension is:

$$
\operatorname{dim}_{\mathbf{C}} V^{(\delta)}=\delta \sum_{\mu_{j}-\mu_{i}=\delta} r_{i} r_{j}
$$

2. The group $V^{(\delta)}$ operates freely on the mid term, which is defined as the cohomology pointed set $\mathcal{F} \leq \delta\left(M_{0}\right):=H^{1}\left(\mathbf{E}_{q}, \Lambda_{I}\left(M_{0}\right) / \Lambda_{I}^{\geq \delta+1}\left(M_{0}\right)\right)$. (The special point of this pointed set is the class of the trivial cocycle all of whose components are the identity.)

3. The corresponding quotient map is the canonical arrow from $\mathcal{F} \leq \delta\left(M_{0}\right)$ to the cohomology pointed set $\mathcal{F}^{\leq \delta-1}\left(M_{0}\right):=H^{1}\left(\mathbf{E}_{q}, \Lambda_{I}\left(M_{0}\right) / \Lambda_{\bar{I}}^{\geq \delta}\left(M_{0}\right)\right)$. 
Thus, the fiber:(17) of $\mathcal{F}^{\leq \delta}\left(M_{0}\right) \rightarrow \mathcal{F}^{\leq \delta-1}\left(M_{0}\right)$ inherit a natural structure of affine space over the vector space $V^{(\delta)}$. Accordingly, for $v \in V^{(\delta)}$, we shall write $\alpha \mapsto v \oplus \alpha$ the translation by $v$ in $\mathcal{F} \leq \delta\left(M_{0}\right)$ (that is, in each of the fibers just mentioned); and for two class $\alpha, \alpha^{\prime} \in \mathcal{F}^{\leq \delta}\left(M_{0}\right)$ having the same image in $\mathcal{F}^{\leq \delta-1}\left(M_{0}\right)$, we shall write $\alpha^{\prime} \ominus \alpha$ the unique element of $V^{(\delta)}$ such that $\alpha^{\prime}=v \oplus \alpha$.

The interpretation of $\mathcal{F} \leq \delta\left(M_{0}\right)$ in terms of classification rests on the same interpolating categories $\mathcal{E}^{\delta}$ as in subsection [2.7. An object of $\mathcal{E}^{\delta}$ can be identified with a matrix $A$ in $\mathrm{GL}_{n}(\mathbf{C}(\{z\}))$, with undetermined blocks $(i, j)$ for $\mu_{j}-\mu_{i}>\delta$, symbolized here by $\star$ :

$$
\left(\begin{array}{cccccc}
z^{\mu_{1}} A_{1} & \ldots & \ldots & \star & \star & \star \\
\ldots & \ldots & \ldots & \ldots & \star & \star \\
\ldots & \ldots & \ldots & U_{i, j} & \ldots & \star \\
0 & \ldots & \ldots & \ldots & \ldots & \ldots \\
\ldots & 0 & \ldots & \ldots & \ldots & \ldots \\
0 & \ldots & 0 & \ldots & \ldots & z^{\mu_{k}} A_{k}
\end{array}\right)
$$

The highest meaningful block diagonal consists in blocks $U_{i, j}$ with level $\mu_{j}-\mu_{i}=\delta$.

We fix a block diagonal matrix $A_{0}$ and we classify all matrices $A$ with diagonal $A_{0}$, up to $q$-Gevrey gauge equivalence of level $>\delta$, that is under transforms in $\mathfrak{G}_{A_{0}}(\mathbf{C}((z)))$ all of whose coefficients are series of $q$-Gevrey level $>\delta$. This amounts to the same as fixing the pure module $M_{0}$ and doing $q$-Gevrey classification in its formal class. The space of isoformal classes above $A_{0}$ in $\mathcal{E}^{\delta}$ received a cohomological description in [22]: it is $\mathcal{F} \leq \delta\left(M_{0}\right)$. Using the Birkhoff-Guenther normal form (loc. cit.), one can moreover require null blocks $(i, j)$ for $\mu_{j}-\mu_{i}>\delta$ and find its dimension as an affine space. We shall write $\operatorname{cl}(A)$ the class of the module $M_{A}$ in $\mathcal{F}^{\leq \delta}\left(A_{0}\right):=\mathcal{F}^{\leq \delta}\left(M_{0}\right)$.

In 3.3, we shall have use for the corresponding computational description of the exact sequence (7) . Consider $A, A^{\prime}$ in $\mathcal{F}^{\leq \delta}\left(M_{0}\right)$ having the same image in $\mathcal{F}^{\leq \delta-1}\left(M_{0}\right)$. Then $\hat{F}_{A, A^{\prime}}:=\hat{F}_{A^{\prime}}\left(\hat{F}_{A}\right)^{-1}$ lies in $\mathfrak{G}_{A_{0}} \geq \delta(\mathbf{C}((z)))$, as well as its

\footnotetext{
$\overline{{ }^{(17)} \text { Actually, each }} \mathcal{F}^{\leq \delta}\left(M_{0}\right)$ can be endowed with an affine structure over the vector space $\bigoplus_{k \leq \delta} V^{(k)}$, but we shall not need this fact.
} 
summations:

$$
S_{\bar{c}} \hat{F}_{A, A^{\prime}}:=S_{\bar{c}} \hat{F}_{A^{\prime}}\left(S_{\bar{c}} \hat{F}_{A}\right)^{-1}
$$

We get a cocycle:

$$
S_{\bar{c}, \bar{d}} \hat{F}_{A, A^{\prime}}:=\left(S_{\bar{c}, \bar{d}} \hat{F}_{A, A^{\prime}}\right)^{-1} S_{\bar{c}, \bar{d}} \hat{F}_{A, A^{\prime}}
$$

of $\Lambda_{I}\left(M_{0}\right)$, in which the blocks for $\mu_{j}-\mu_{i}>\delta$ have no meaning and those for $0<\mu_{j}-\mu_{i}<\delta$ vanish; thus, it yields a well defined privileged cocycle of $\lambda_{I}\left(M_{0}\right)^{(\delta)}:=\Lambda_{\bar{I}}^{\geq \delta}\left(M_{0}\right) / \Lambda_{\bar{I}}^{\geq \delta+1}\left(M_{0}\right)$, whence a class in $V^{(\delta)}:=H^{1}\left(\mathbf{E}_{q}, \lambda_{I}^{(\delta)}\left(M_{0}\right)\right)$. This class is the element $\operatorname{cl}\left(A^{\prime}\right) \ominus \operatorname{cl}(A) \in V^{(\delta)}$ which sends the class of $A$ to the class of $A^{\prime}$ in $\mathcal{F}^{\leq \delta}\left(M_{0}\right)$.

\subsection{Linking representations of $\mathfrak{s t}$ to isoformal analytic classes. -}

Let $M_{0}$ an object of $\mathcal{E}_{p, 1}^{(0)}$ Its fiber by the functor gr from $\mathcal{E}_{1}^{(0)}$ to $\mathcal{E}_{p, 1}^{(0)}$ can be identified with the category $\mathcal{C}\left(M_{0}\right)$ with objects the pairs $(M, u), M$ an object of $\mathcal{E}_{1}^{(0)}$ and $u: \operatorname{gr} M \rightarrow M_{0}$ an isomorphism; and with morphisms $(M, u) \rightarrow(N, v)$ the morphisms $f: M \rightarrow N$ in $\mathcal{E}_{1}^{(0)}$ such that $u=v \circ \operatorname{gr} f$. Such a morphism is automatically an isomorphism so that $\mathbf{C}\left(M_{0}\right)$ is a groupoid and $\mathcal{F}\left(M_{0}\right)$ is the set $\pi_{0}\left(\mathbf{C}\left(M_{0}\right)\right)$ of its connected components. Its cohomological description was explained in 3.1, we now use tannakian duality to get a representation theoretic description.

To alleviate notations, in this section, we respectively write $\mathcal{C}, \mathcal{C}_{0}$ for $\mathcal{E}_{1}^{(0)}, \mathcal{E}_{p, 1}^{(0)}$ and $G, G_{0}$ for their Galois groups $G_{1}^{(0)}=\operatorname{Gal}\left(\mathcal{E}_{1}^{(0)}\right), G_{p, 1}^{(0)}=\operatorname{Gal}\left(\mathcal{E}_{p, 1}^{(0)}\right)$. We write $\operatorname{Rep}_{\mathbf{C}}(G), \operatorname{Rep}_{\mathbf{C}}\left(G_{0}\right)$ the categories of complex finite dimensional rational representations of these proalgebraic groups. The choice of the fiber functors is here irrelevant, all that we need is the equivalences of category $\mathcal{C}$ with $\operatorname{Rep}_{\mathbf{C}}(G)$ and of category $\mathcal{C}_{0}$ with $\operatorname{Rep}_{\mathbf{C}}\left(G_{0}\right)$. Objects of $\operatorname{Rep}_{\mathbf{C}}(G)$ are rational morphisms $\rho: G \rightarrow \mathrm{GL}(V), V$ a finite dimensional complex space; and morphisms in $\operatorname{Rep}_{\mathbf{C}}(G)$ from $\rho: G \rightarrow \operatorname{GL}(V)$ to $\rho^{\prime}: G \rightarrow \operatorname{GL}\left(V^{\prime}\right)$ are linear maps $\phi: V \rightarrow V^{\prime}$ such that $\forall g \in G, \rho^{\prime}(g) \circ \phi=\phi \circ \rho(g)$. The category $\operatorname{Rep}_{\mathbf{C}}\left(G_{0}\right)$ is similarly defined.

We also introduce the auxiliary comma-category $\overline{\mathcal{C}}$ with objects the triples $\left(M, M_{0}, u\right)$ where $M, M_{0}$ are objects of $\mathcal{C}, \mathcal{C}_{0}$ and where $u: \operatorname{gr} M \rightarrow M_{0}$ is an isomorphism; and with morphisms $\left(M, M_{0}, u\right) \rightarrow\left(N, N_{0}, v\right)$ the pairs $\left(f, f_{0}\right)$ made up of a morphism $f: M \rightarrow N$ and of a morphism $f_{0}: M_{0} \rightarrow N_{0}$ such that $f_{0} \circ u=v \circ \operatorname{gr} f$. 
Lemma 3.2. - The category $\overline{\mathcal{C}}$ is equivalent to $\mathcal{C}$ and we can identify the fiber $\mathcal{C}\left(M_{0}\right)$ described above with the fiber $\overline{\mathcal{C}}\left(M_{0}\right)$.

Proof. - Let $F$ be the functor from $\mathcal{C}$ to $\overline{\mathcal{C}}$ defined by $M \leadsto\left(M, \operatorname{gr} M, \operatorname{Id}_{\operatorname{gr} M}\right)$ and $f \leadsto(f, \operatorname{gr} f)$ and let $G$ be the forgetful functor from $\overline{\mathcal{C}}$ to $\mathcal{C}$. Then $G \circ F$ is the identity functor of $\mathcal{C}$ and $F \circ G$ is isomorphic to the identity functor of $\overline{\mathcal{C}}$ by the natural transformation which sends $X=\left(M, M_{0}, u\right)$ to the morphism $\left(\operatorname{Id}_{M}, u\right)$ from $F \circ G(X)=\left(M, \operatorname{gr} M, \operatorname{Id}_{\operatorname{gr} M}\right)$ to $X$.

We now carry on this construction to the equivalent categories $\operatorname{Rep}_{\mathbf{C}}(G)$ and $\operatorname{Rep}_{\mathbf{C}}\left(G_{0}\right)$. The inclusion $\mathcal{C}_{0} \hookrightarrow \mathcal{C}$ and its retraction gr : $\mathcal{C} \rightarrow \mathcal{C}_{0}$ correspond dually to morphisms $G_{0} \stackrel{\mathrm{gr}^{*}}{\rightarrow} G \stackrel{\pi}{\rightarrow} G_{0}$ such that $\pi \circ \mathrm{gr}^{*}=\operatorname{Id}_{G_{0}}$. We shall use gr* to identify $G_{0}$ to a (proalgebraic) subgroup of $G$. The Stokes group is $\mathfrak{S t}=\operatorname{Ker} \pi$ and one deduces from these morphisms the equality $G=\mathfrak{S} \mathfrak{t} \rtimes G_{0}$. Thus, $G_{0}$ acts upon $\mathfrak{S t}$ by inner automorphisms, which we shall denote $s \mapsto s^{g}:=g^{-1} s g$. We also shall denote $D \mapsto D^{g}$ the corresponding adjoint action on the Lie algebra $\mathfrak{s t}$ of $\mathfrak{S t}$.

The functor $\mathcal{C}_{0} \hookrightarrow \mathcal{C}$ is thereby identified with the following functor from $\operatorname{Rep}_{\mathbf{C}}\left(G_{0}\right)$ to $\operatorname{Rep}_{\mathbf{C}}(G)$ :

$$
\begin{aligned}
\left(\rho_{0}: G_{0} \rightarrow \operatorname{GL}(V)\right) & \sim\left(\rho_{0} \circ \pi: G \rightarrow \operatorname{GL}(V)\right), \\
\left(\phi: V \rightarrow V^{\prime}, \rho_{0} \rightarrow \rho_{0}^{\prime}\right) & \sim\left(\phi: V \rightarrow V^{\prime}, \rho_{0} \circ \pi \rightarrow \rho_{0}^{\prime} \circ \pi\right) .
\end{aligned}
$$

Similarly, the functor gr : $\mathcal{C} \rightarrow \mathcal{C}_{0}$ is identified with the following functor from $\operatorname{Rep}_{\mathbf{C}}(G)$ to $\operatorname{Rep}_{\mathbf{C}}\left(G_{0}\right)$ :

$$
\begin{aligned}
(\rho: G \rightarrow \mathrm{GL}(V)) & \sim\left(\rho_{\mid G_{0}}: G_{0} \rightarrow \mathrm{GL}(V)\right), \\
\left(\phi: V \rightarrow V^{\prime}, \rho \rightarrow \rho^{\prime}\right) & \sim\left(\phi: V \rightarrow V^{\prime}, \rho_{\mid G_{0}} \rightarrow \rho_{\mid G_{0}}^{\prime}\right) .
\end{aligned}
$$

Since $\left(\rho_{0} \circ \pi\right)_{\mid G_{0}}=\rho_{0}$, the composition is the identity of $\operatorname{Rep}_{\mathbf{C}}\left(G_{0}\right)$ as it should. Then one checks that $\overline{\mathcal{C}}$ is identified to the category of triples $\left(\rho, \rho_{0}, u\right)$, where $\rho: G \rightarrow \mathrm{GL}(V)$ and $\rho_{0}: G_{0} \rightarrow \mathrm{GL}\left(V_{0}\right)$ are rational representations and where $u: V \rightarrow V_{0}$ is an isomorphism from $\rho_{\mid G_{0}}$ to $\rho_{0}$, with morphisms from $\left(\rho, \rho_{0}, u\right)$ to $\left(\rho^{\prime}, \rho_{0}^{\prime}, u^{\prime}\right)$ the pairs $\left(\phi, \phi^{\prime}\right)$ where $\phi: V \rightarrow V^{\prime}$ and $\phi^{\prime}: v^{\prime} \rightarrow V_{0}^{\prime}$ yield morphisms $\rho \rightarrow \rho^{\prime}$ and $\rho_{0} \rightarrow \rho_{0}^{\prime}$ of representations and where moreover $\phi_{0} \circ u=u^{\prime} \circ \phi$. The equivalences of $\mathcal{C}$ and $\overline{\mathcal{C}}$ are easy to explicit.

Last, if $M_{0}$ "is" the representation $\rho_{0}: G_{0} \rightarrow \mathrm{GL}(V)$, the fiber $\overline{\mathcal{C}}\left(M_{0}\right)$ is identified with the category with objects the pairs $(\rho, u)$ of a rational representation $\rho: G \rightarrow \operatorname{GL}(V)$ and a map $u: V \rightarrow V_{0}$ which is an isomorphism 
from $\rho_{\mid G_{0}}$ to $\rho_{0}$, with morphisms from $(\rho, u)$ to $\left(\rho^{\prime}, u^{\prime}\right)$ the maps $\phi: V \rightarrow V^{\prime}$ which yield morphisms $\rho \rightarrow \rho^{\prime}$ such that $u=u^{\prime} \circ \phi$.

Lemma 3.3. - The fiber $\overline{\mathcal{C}}\left(M_{0}\right)$ can be identified with the set of representations $\rho: G \rightarrow G L\left(V_{0}\right)$ such that $\rho_{\mid G_{0}}=\rho_{0}$.

Proof. - This set is considered as a category having only identity morphisms. The identification comes from the functor which sends the object $(\rho, u)$ to the representation $\rho_{u}: g \mapsto u \circ \rho(g) \circ u^{-1}$ and every morphism to the corresponding identity morphism. This is a retraction of the obvious inclusion, and an equivalence of categories.

Now we return to our more concrete setting, with $G=\mathfrak{S t} \rtimes G_{0}$. If $\rho_{0}$ : $G_{0} \rightarrow \mathrm{GL}(V)$ is fixed, to specify a representation $\rho: G \rightarrow \mathrm{GL}\left(V_{0}\right)$ such that $\rho_{\mid G_{0}}=\rho_{0}$, we need only to give its restriction $\bar{\rho}$ to $\mathfrak{S t}$, and this is subject to the necessary and sufficient condition:

$$
\forall s \in \mathfrak{S t}, \forall g \in G_{0}, \bar{\rho}\left(s^{g}\right)=(\bar{\rho}(s))^{\rho_{0}(g)} .
$$

Since $\mathfrak{S t}$ is connected and prounipotent, $\bar{\rho}$ is determined by the corresponding representation of the Lie algebra $\mathfrak{s t}$. In the end, we have proved:

Proposition 3.4. - The fiber $\overline{\mathcal{C}}\left(M_{0}\right)$ can be identified with the set:

$$
\left\{\rho: \mathfrak{s t} \rightarrow g l\left(V_{0}\right) \mid \forall D \in \mathfrak{s t}, \forall g \in G_{0}, \rho\left(D^{g}\right)=(\rho(D))^{\rho_{0}(g)}\right\} .
$$

3.3. Linking representations of st with $H^{1}\left(\mathbf{E}_{q}, \lambda_{I}\left(M_{0}\right)\right)$. - The bijection of $H^{1}\left(\mathbf{E}_{q}, \lambda_{I}\left(M_{0}\right)\right)$ with $\operatorname{Rep}_{A_{0}}(\tilde{\mathfrak{s t}})$ resulting from the two descriptions of $\mathcal{F}\left(M_{0}\right)$ (see sections 2.4, 3.1 and 3.2) is obtained as follows: for any matrix $A$ corresponding to a class in $\mathcal{F}\left(M_{0}\right)$, first compute the privileged cocycle $\left(S_{\bar{c}, \bar{d}} \hat{F}_{A}\right) \in Z_{p r}^{1}\left(\mathfrak{U}_{A_{0}}, \Lambda_{I}\left(M_{0}\right)\right)$. Write temporarily $h(A)$ its class in $H^{1}\left(\mathbf{E}_{q}, \lambda_{I}\left(M_{0}\right)\right)$. On the other hand, write $D_{\alpha}$ the residue at $\beta=\alpha$ of the meromorphic function $\beta \mapsto \log S_{\overline{0_{0}, \beta}} \hat{F}_{A}\left(z_{0}\right) \in \mathfrak{g}_{A_{0}}$, and $\Delta_{\alpha}^{(\delta, \bar{c})}(A)$ the components of $D_{\alpha}$ for $\alpha^{\delta}=\bar{c}$. Then call $\rho(A) \in \operatorname{Rep}_{A_{0}}(\tilde{\mathfrak{s t}})$ the unique representation which sends the " $q$-alien derivations" $\Delta_{\alpha}^{(\delta, \bar{c})}$ to the $\Delta_{\alpha}^{(\delta, \bar{c})}(A)$ and $\nu$ to $U$ (the block-diagonal matrix of unipotent components of $A$ ). The bijection puts in correspondance the class $h(A)$ with the representation $\rho(A)$. 
Using $Z_{p r}^{1}\left(\mathfrak{U}_{A_{0}},\left(\Lambda_{I} / \Lambda_{\bar{I}}^{>\delta+1}\right)\left(M_{0}\right)\right)$, we get correspondingly a bijection of $H^{1}\left(\mathbf{E}_{q},\left(\Lambda_{I} / \Lambda_{\bar{I}}^{\geq \delta+1}\right)\left(M_{0}\right)\right)$ with $\operatorname{Rep}_{A_{0}}\left(\tilde{\mathfrak{s t}}^{\leq \delta}\right)$. This gives a commutative diagram with surjective horizontal arrows and bijective vertical arrows:

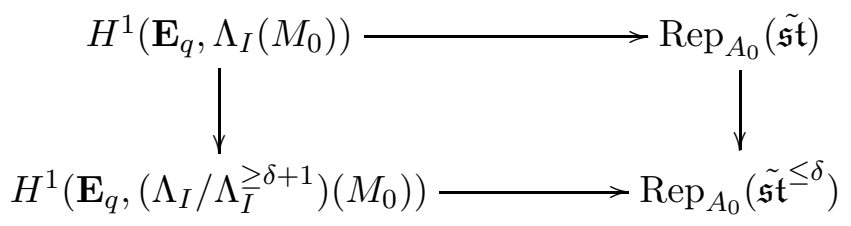

Just with this information, we shall now start to get structural information about $\tilde{\mathfrak{s t}}$. Let $c \in \mathbf{C}^{*}, \delta \in \mathbf{N}^{*}$ and set $A_{0}:=\left(\begin{array}{cc}1 & 0 \\ 0 & c z^{\delta}\end{array}\right)$. Then:

$$
\mathfrak{g}_{A_{0}}=\mathfrak{g}_{A_{0}}{ }^{(\delta, \bar{c})}=\left(\begin{array}{cc}
0 & \mathbf{C} \\
0 & 0
\end{array}\right) .
$$

Since $\mathfrak{g}_{A_{0}}$ is abelian, $\operatorname{Rep}_{A_{0}}(\tilde{\mathfrak{s} t})$ can be identified with the dual space of $\left(\frac{\tilde{\mathfrak{s t}}}{[\tilde{\mathfrak{s} t, \tilde{\mathfrak{s} t}]}}\right)^{(\delta, \bar{c})}$. But since $H^{1}\left(\mathbf{E}_{q}, \Lambda_{I}\left(M_{0}\right)\right)=H^{1}\left(\mathbf{E}_{q}, \mathcal{F}_{c z^{\delta}}\right)$ has dimension $\delta$ 22, 20, we conclude:

$$
\operatorname{dim}_{\mathbf{C}}\left(\frac{\tilde{\mathfrak{s t}}}{[\tilde{\mathfrak{s t}}, \tilde{\mathfrak{s} \mathfrak{t}]}}\right)^{(\delta, \bar{c})}=\delta
$$

Applying theorem 2.8, we see that (the images of) the $\Delta_{\alpha}^{(\delta, \bar{c})}$ for arbitrary $\alpha \in$ $\mathbf{E}_{q}$ generate the complex vector space $\left(\frac{\tilde{\mathfrak{s t}}}{[\mathfrak{s t}, \tilde{\mathfrak{s t} t})}\right)^{(\delta, \bar{c})}$. However, when computing the residues in the case of a matrix $A_{u}:=\left(\begin{array}{cc}1 & u \\ 0 & c z^{\delta}\end{array}\right)$, we only find poles at points such that $\alpha^{\delta}=\bar{c}$. Thus, if all the $\Delta_{\alpha}^{(\delta, \bar{c})}$ such that $\alpha^{\delta}=\bar{c}$ vanish on the class of $A_{u}$, this class is trivial. By duality, this means that (the images of) those $\Delta_{\alpha}^{(\delta, \bar{c})}$ such that $\alpha^{\delta}=\bar{c}$ generate $\left(\frac{\tilde{\mathfrak{s t}}}{[\tilde{s t, j \mathfrak{s t}]}}\right)^{(\delta, \bar{c})}$.

Remark 3.5. - This is in essence the content of [20, theorem 4.9] and represent the basic step in the "abelian (two slopes) case". In [21 we attempted to obtain the general case by devissage of the filtration. Here we achieve this devissage in the next two subsections.

For each pair $(\delta, \bar{c}) \in \mathbf{N}^{*} \times \mathbf{E}_{q}$, we choose $\delta$ among the $\delta^{2}$ points $\alpha \in \mathbf{E}_{q}$ such that $\alpha^{\delta}=\bar{c}$ in such a way that the images of the corresponding $\Delta_{\alpha}^{(\delta, \bar{c})}$ form a basis of $\frac{\tilde{\mathfrak{s t}}}{[\tilde{\mathfrak{s t}}, \tilde{\mathfrak{s t}}]}$. We write $\Delta_{i}^{(\delta, \bar{c})}, 1 \leq i \leq \delta$, the corresponding $q$-alien derivations. In accordance with the analogy explained in the introduction, 
we see them as "pointed" and from now on they will be denoted $\dot{\Delta}_{i}^{(\delta, \bar{c})}$, and $\nu \in \mathfrak{s t}^{(0)}$ will be denoted $\dot{\Delta}^{(0)}$.

Proposition 3.6. - The family of all $\dot{\Delta}_{i}^{(\delta, \bar{c})}$ together with $\dot{\Delta}^{(0)}:=\nu$ topologically generate $\tilde{\mathfrak{s t}}$.

Proof. - Call $S$ the sub-Lie algebra generated by this family. It is naturally $\mathbf{N}$-graded and one has, for all $\delta \in \mathbf{N}$ :

$$
\tilde{\mathfrak{s t}}^{(\delta)}=S^{(\delta)}+[\tilde{\mathfrak{s t}}, \tilde{\mathfrak{s t}}]^{(\delta)}=S^{(\delta)}+\sum_{i+j=\delta}\left[\tilde{\mathfrak{s t}}^{(i)}, \tilde{\mathfrak{s t}}^{(j)}\right]
$$

We shall prove inductively that $S^{(\delta)}=\tilde{\mathfrak{s t}}^{(\delta)}$ for all $\delta \in \mathbf{N}$, which will imply the conclusion. For $\delta=0$, both sides are equal to $\mathbf{C} \nu$. Assuming it to be true for all degrees $<\delta$, we calculate:

$$
\begin{aligned}
\tilde{\mathfrak{s t}}^{(\delta)} & =S^{(\delta)}+\sum_{i+j=\delta}\left[\tilde{\mathfrak{s t}}^{(i)}, \tilde{\mathfrak{s t}}^{(j)}\right] \\
& =S^{(\delta)}+\left[\mathbf{C} \nu, \tilde{\mathfrak{s t}}^{(\delta)}\right]+\sum_{\substack{i+j=\delta \\
i, j<\delta}}\left[\tilde{\mathfrak{s t}}^{(i)}, \tilde{\mathfrak{s t}}^{(j)}\right] \\
& =S^{(\delta)}+\left[\mathbf{C} \nu, \tilde{\mathfrak{s t}}^{(\delta)}\right]+\sum_{\substack{i+j=\delta \\
i, j<\delta}}\left[S^{(i)}, S^{(j)}\right] \\
& =S^{(\delta)}+\left[\mathbf{C} \nu, \tilde{\mathfrak{s t}}^{(\delta)}\right]
\end{aligned}
$$

since $\left[S^{(i)}, S^{(j)}\right] \subset S^{(\delta)}$ when $i+j=\delta$. Iterating, we get $\tilde{\mathfrak{s t}}^{(\delta)}=S^{(\delta)}+\Phi^{k}\left(\tilde{\mathfrak{s t}}^{(\delta)}\right)$ for all $k$, where $\Phi(E):=[\mathbf{C} \nu, E]$. By the topological description of $\tilde{\mathfrak{s t}}$ at the end of 2.1, this ends the proof.

We will show in 3.5 that this family is in some sense free.

3.4. q-Gevrey devissage of the space of representations. - From the $q$-Gevrey dévissage of $H^{1}\left(\mathbf{E}_{q}, \Lambda_{I}\left(M_{0}\right)\right)$ and of $Z_{p r}^{1}\left(\mathfrak{U}_{A_{0}}, \Lambda_{I}\left(M_{0}\right)\right)$, and from the identifications with $\operatorname{Rep}_{A_{0}}(\tilde{\mathfrak{s}})$, we get the following commutative diagram of 
exact sequences (for concision, we do not indicate the dependency on $M_{0}$ ):

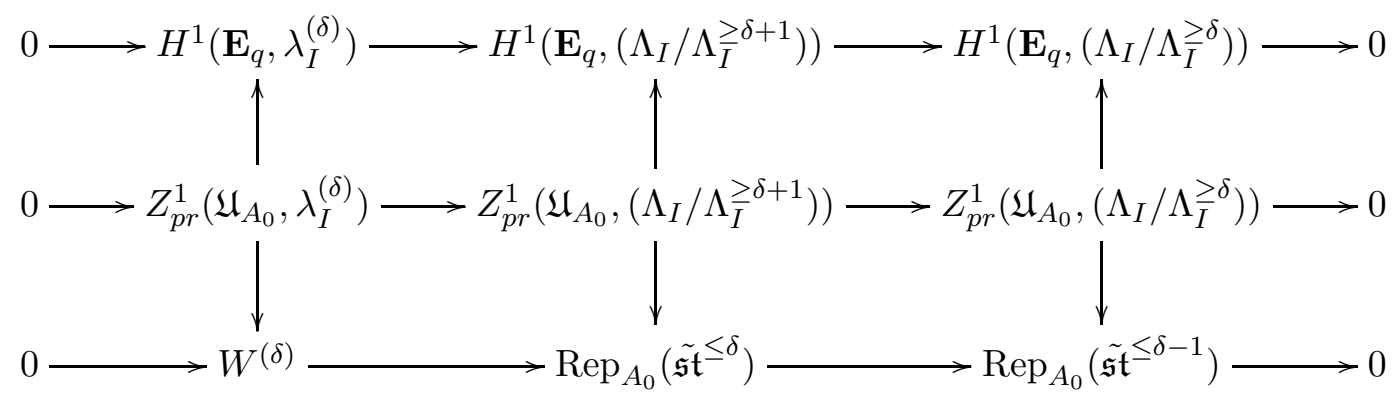

In the last line, being an exact sequence means that $W^{(\delta)}$ is a vector space acting on the middle term with quotient the rightmost term. We shall now describe this space and this action. For this, we recall the description given in [21, prop. 3.3.9] of the fibers of the surjection from $\operatorname{Rep}_{A_{0}}\left(\tilde{\mathfrak{s t}}^{\leq \delta}\right)$ to $\operatorname{Rep}_{A_{0}}\left(\tilde{\mathfrak{s t}}^{\leq \delta-1}\right.$ ) (modulo the change of notation from $\mathfrak{s t}(\delta)$ there to $\tilde{\mathfrak{s t}}^{\leq \delta}$ here). Let $B$ an element of $\mathcal{C}^{\delta-1}$ in Birkhoff-Guenther normal form having graded part $A_{0}$. Two elements $A, A^{\prime}$ of $\mathcal{C}^{\delta}$ lifting $B$ are related by a unique formal gauge transform $\hat{F}_{A, A^{\prime}} \in \mathfrak{G}_{A_{0}}(\mathbf{C}((z)))$. This matrix has null blocks for $0<\mu_{j}-\mu_{i}<\delta$; the blocks corresponding to levels $\mu_{j}-\mu_{i}>\delta$ are irrelevant; and we call $\hat{f}_{A, A^{\prime}}$ the part of $\hat{F}_{A, A^{\prime}}$ corresponding to level $\mu_{j}-\mu_{i}=\delta$. The family of all the $S_{\bar{c}, \bar{d}} \hat{f}_{A, A^{\prime}}$ is a cocycle in $Z_{p r}^{1}\left(\mathfrak{U}_{A_{0}}, \lambda_{I}^{(\delta)}\right)$. Then:

$$
\Delta_{\alpha}^{(\delta)}\left(A, A^{\prime}\right):=\Delta_{\alpha}^{(\delta)}\left(A^{\prime}\right)-\Delta_{\alpha}^{(\delta)}(A)=\operatorname{Res}_{\beta=\alpha} S_{\overline{c_{0}}, \beta} \hat{f}_{A, A^{\prime}}\left(z_{0}\right)
$$

Moreover, all families $\left(\Delta_{\alpha}^{(\delta)}\right)$ arising this way correspond to a difference $\Delta\left(A^{\prime}\right)$ $\Delta(A)$. We thus define:

$$
W^{(\delta)}:=\left\{\left(\operatorname{Res}_{\beta=\alpha} S_{\overline{\bar{c}_{0}}, \beta} f\left(z_{0}\right)\right)_{\alpha}^{(\delta, \bar{c})} \mid f \in Z_{p r}^{1}\left(\mathfrak{U}_{A_{0}}, \lambda_{I}^{(\delta)}\right)\right\} .
$$

If we encode a representation by the family of all the $\dot{\Delta}_{i}^{(\delta, \bar{c})}(A)$, we see that we do get an action of $W^{(\delta)}$ on $\operatorname{Rep}_{A_{0}}\left(\tilde{\mathfrak{s t}}^{\leq \delta}\right)$ with quotient $\operatorname{Rep}_{A_{0}}\left(\tilde{\mathfrak{s t}}^{\leq \delta-1}\right)$.

\subsection{Freeing the alien derivations. -}

Definition 3.7. - Let $\dot{\Delta}^{(0)}$ be a symbol corresponding to to the element $\nu$ introduced in section 2.4. For each $(\delta, \bar{c}) \in \mathbf{N}^{*} \times \mathbf{E}_{q}$ and $i=1, \ldots, \delta$, let $\dot{\Delta}_{i}^{(\delta, \bar{c})}$ be a symbol corresponding to the actual alien derivation with the same notation. We call $L$ the free Lie algebra generated by $\dot{\Delta}^{(0)}$ and all the $\dot{\Delta}_{i}^{(\delta, \bar{c})}$. We graduate it by the semi-group $\{0\} \cup \mathbf{N}^{*} \times \mathbf{E}_{q}$ by taking $\operatorname{deg} \dot{\Delta}^{(0)}=0$ and 
$\operatorname{deg} \dot{\Delta}_{i}^{(\delta, \bar{c})}=(\delta, \bar{c})$. We also endow $L$ with the following action of $T_{1}^{(0)} \times G_{f, 1}^{(0)}$ :

$$
\begin{aligned}
\left(\dot{\Delta}_{i}^{(\delta, \bar{c})}\right)^{(t, \gamma)} & :=t^{\delta} \gamma(\bar{c}) \dot{\Delta}_{i}^{(\delta, \bar{c})} \\
\left(\dot{\Delta}^{(0)}\right)^{(t, \gamma)} & :=\dot{\Delta}^{(0)}
\end{aligned}
$$

We write $\operatorname{Rep}_{A_{0}}(L)$ the set of representations from $L$ to $\mathfrak{g}_{A_{0}}$ compatible with this action (and similarly for all stable sub-Lie algebras of $L$ ).

Write $L^{\leq \delta}$ the sub-Lie algebra generated by $\dot{\Delta}^{(0)}$ and all the $\dot{\Delta}_{i}^{\left(\delta^{\prime}, \bar{c}\right)}, \delta^{\prime} \leq \delta$; and $L^{>\delta}$ the ideal generated by all the $\dot{\Delta}_{i}^{\left(\delta^{\prime}, \bar{c}\right)}, \delta^{\prime}>\delta$. After [4, chap. 2, $\S 2$, no 9 , prop. 10], $L \leq \delta$ is free with basis the stated system of generators, while $L^{>\delta}$ is free with basis the family of all $\left(a d D_{1}\right) \cdots\left(a d D_{k}\right) \dot{\Delta}_{i}^{\left(\delta^{\prime \prime}, \bar{c}\right)}$ where the $D_{i}$ are $\dot{\Delta}_{i}^{\left(\delta^{\prime}, \bar{c}\right)}$ with $\delta^{\prime} \leq \delta$ and where $\delta^{\prime \prime}>\delta$. Moreover, $L=L^{\leq \delta} \oplus L^{>\delta}$. Likewise, we have $L^{\leq \delta}=L^{\leq \delta-1} \oplus L^{(\delta)}$, where $L^{(\delta)}$ is the ideal generated by all the $\dot{\Delta}_{i}^{(\delta, \bar{c})}$.

From the obvious dominant morphisms of Lie algebras $L \rightarrow \tilde{\mathfrak{s t}}$ and $L \leq \delta \rightarrow$ $\tilde{\mathfrak{s t}^{\prime}} \leq \delta$, one deduces a commutative diagram with surjective horizontal maps and injective vertical maps:

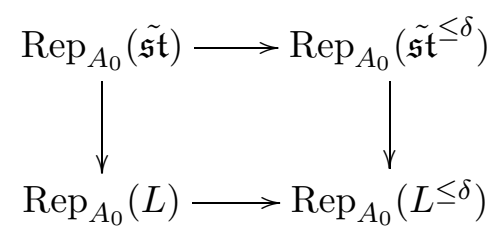

On the other hand, we have identifications:

$$
\begin{gathered}
U:=\operatorname{Rep}_{A_{0}}(L) \approx \bigoplus_{\left(\delta, \bar{c} \in \mathbf{N}^{*} \times \mathbf{E}_{q}\right.}\left(\mathfrak{g}_{A_{0}}{ }^{(\delta, \bar{c})}\right)^{\delta} \\
U^{\leq \delta}:=\operatorname{Rep}_{A_{0}}\left(L^{\leq \delta}\right) \approx \bigoplus_{\substack{\left(\delta^{\prime}, \bar{c}\right) \in \mathbf{N}^{*} \times \mathbf{E}_{q} \\
1 \leq \delta^{\prime} \leq \delta}}\left(\mathfrak{g}_{A_{0}}{ }^{\left(\delta^{\prime}, \bar{c}\right)}\right)^{\delta^{\prime}} \\
U^{(\delta)}:=\operatorname{Rep}_{A_{0}}\left(L^{(\delta)}\right) \approx \bigoplus_{\bar{c} \in \mathbf{E}_{q}}\left(\mathfrak{g}_{A_{0}}(\delta, \bar{c})\right)^{\delta} .
\end{gathered}
$$

Indeed, the value of the generator $\nu$ is imposed since we consider representations in $\operatorname{Rep}_{A_{0}}$, i.e. relative to the fixed $A_{0}$. Then, we can enrich as follows 
the previous diagram of exact sequences:

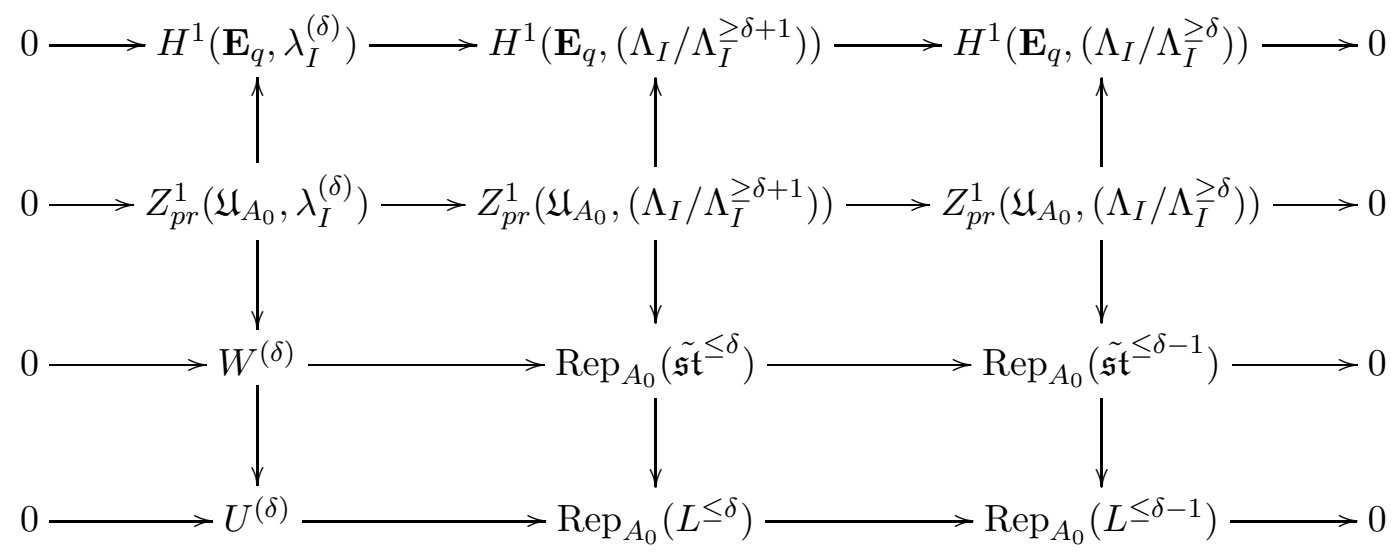

The new vertical arrows are a priori injections.

Theorem 3.8 (Freeness theorem). - The map $\operatorname{Rep}_{A_{0}}(\tilde{\mathfrak{s t}}) \rightarrow \operatorname{Rep}_{A_{0}}(L)$ is bijective.

Proof. - By induction, using the two last lines of the above diagram, it is enough to show that the leftmost vertical arrow is bijective. But it is linear and it sends injectively each $W^{(\delta, \bar{c})}$ to $U^{(\delta, \bar{c})}$, which has the same dimension $\delta$.

3.6. First step in direction of the inverse problem. - Recall from section 2.2 the description of the pure (or formal) Galois group with integral slopes:

$$
G_{p, 1}^{(0)}=\operatorname{Gal}\left(\mathcal{E}_{p, 1}^{(0)}\right)=\mathbf{C}^{*} \times \operatorname{Hom}_{g r}\left(\mathbf{C}^{*} / q^{\mathbf{Z}}, \mathbf{C}^{*}\right) \times \mathbf{C} .
$$

In section 2.4, we took off its unipotent component $\mathbf{C}$ and glued it with the Stokes group, so we now introduce its semi-simple component:

$$
G_{p, 1, s}^{(0)}:=\mathbf{C}^{*} \times \operatorname{Hom}_{g r}\left(\mathbf{C}^{*} / q^{\mathbf{Z}}, \mathbf{C}^{*}\right) .
$$

It acts as follows on the free Lie algebra $L$ : the action on $\mathbf{C} \nu$ is trivial; for each $(\delta, \bar{c}) \in \mathbf{N}^{*} \times \mathbf{E}_{q}$, the action on the component $L^{(\delta, \bar{c})}$ is multiplication by $t^{\delta} \gamma(\bar{c})$.

Definition 3.9. - The wild fundamental group of $\mathcal{E}_{1}^{(0)}$ is the semi-direct product $L \rtimes G_{p, 1, s}^{(0)}$. A representation of the wild fundamental group is the data of a rational linear representation of $G_{p, 1, s}^{(0)}$ together with a representation of $L$, required to be compatible with the corresponding adjoint actions. 
The Tannakian category $\operatorname{Rep}\left(L \rtimes G_{p, 1, s}^{(0)}\right)$ is by definition built with such representations.

Let $\rho: L \rtimes G_{p, 1, s}^{(0)} \rightarrow \mathrm{GL}(V)$ be a representation of the wild fundamental group in the sense of the above definition. It it is easy to check that the restriction $d \rho_{2}: L \rightarrow \operatorname{End}(V)$ is nilpotent and vanishes on every alien derivation but perhaps a finite number. Therefore $d \rho_{2}$ factors by $L^{\dagger}, L^{\dagger}$ being the fpronilpotent completion of the free Lie algebra $L$ ( $c f$. the appendix $\mathrm{A}$ ), which is a pronilpotent proalgebraic Lie algebra.

Remark 3.10. - The natural morphism $L \rightarrow \tilde{\mathfrak{s t}}$ factors into $L \rightarrow L^{\dagger} \rightarrow \tilde{\mathfrak{s t}}$, the first morphism being injective and dominant (i.e. its image is dense) We deduce morphisms:

$$
L \rtimes G_{p, 1, s}^{(0)} \rightarrow L^{\dagger} \rtimes G_{p, 1, s}^{(0)} \rightarrow \tilde{\mathfrak{s t}} \rtimes G_{p, 1, s}^{(0)},
$$

and then functors:

$$
\operatorname{Rep}\left(\tilde{\mathfrak{s t}} \rtimes G_{p, 1, s}^{(0)}\right) \rightarrow \operatorname{Rep}\left(L^{\dagger} \rtimes G_{p, 1, s}^{(0)}\right) \rightarrow \operatorname{Rep}\left(L \rtimes G_{p, 1, s}^{(0)}\right) .
$$

It follows from theorem 3.8 that these are equivalences of categories, therefore:

$$
L^{\dagger} \rtimes G_{p, 1, s}^{(0)} \rightarrow \tilde{\mathfrak{s t}} \rtimes G_{p, 1, s}^{(0)}
$$

is an isomorphism in the obvious proalgebraic sense (proposition A.3) and $L^{\dagger} \rightarrow \tilde{\mathfrak{s t}}$ is an isomorphism of pronilpotent proalgebraic Lie algebras.

Finally we get an isomorphism of proalgebraic groups:

$$
\exp \left(L^{\dagger}\right) \rtimes G_{p, 1, s}^{(0)} \rightarrow \exp (\tilde{\mathfrak{s t}}) \rtimes G_{p, 1, s}^{(0)}=\mathfrak{S t} \rtimes G_{p, 1}^{(0)}=G_{1}^{(0)} .
$$

This is an "explicit description" of the tannakian group $G_{1}^{(0)}$.

To summarize, we have proved:

Theorem 3.11. - There is a natural bijection between representations of the wild fundamental group of $\mathcal{E}_{1}^{(0)}$ and isomorphism classes of objects of $\mathcal{E}_{1}^{(0)}$. All the Galois groups of such objects are images of such representations.

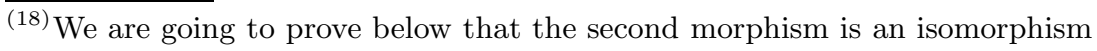




\section{Structure of the global Galois group}

We consider here the Galois theory of equations with matrix in $\mathrm{GL}_{n}(\mathbf{C}(z))$. We shall not develop the theory in such general terms as we did in the previous sections, but just enough to be able to apply it to the inverse problem.

4.1. The global fuchsian Galois group. - We recall here results from [30], mostly its subsection 3.2. Unhappily, some of the results that we need are not completely proven there: details can be found in the thesis "Théorie de Galois des équations aux q-différences fuchsiennes", available at http://www.math.univ-toulouse.fr/ ${ }^{\sim}$ sauloy/PAPIERS/these.pdf. We shall slightly adapt the notations of loc. cit. so that they extend more easily to our case of interest in the next section.

Let $\mathcal{E}_{f}$ be the category with objects the matrices $A \in \mathrm{GL}_{n}(\mathbf{C}(z))$ which are fuchsian (19) at 0 and at $\infty$, and with morphisms $F: A \rightarrow B$ the matrices $F \in \operatorname{Mat}_{p, n}(\mathbf{C}(z))$ such that $\left(\sigma_{q} F\right) A=B F$. It is endowed with a natural tensor structure $(20)$ which makes it a neutral tannakian category. Each object $A$ of $\mathcal{E}_{f}$ can be written, non canonically:

$$
A=M^{(0)}\left[A^{(0)}\right]=M^{(\infty)}\left[A^{(\infty)}\right],
$$

where:

$$
A^{(0)}, A^{(\infty)} \in \mathrm{GL}_{n}(\mathbf{C}) \text { and } M^{(0)} \in \mathrm{GL}_{n}(\mathbf{C}(\{z\})), M^{(\infty)} \in \mathrm{GL}_{n}(\mathbf{C}(\{w\})) .
$$

For the constant matrix $C \in \mathrm{GL}_{n}(\mathbf{C})$, one builds a canonical fundamental solution of $\sigma_{q} X=C X$ in the following way. First, special functions are built from theta functions that satisfy the following elementary equations: $\sigma_{q} l_{q}=l_{q}+1$; and, for all $c \in \mathbf{C}^{*}: \sigma_{q} e_{c}=c e_{c}$. All these functions are meromorphic over $\mathbf{C}^{*}$; moreover, we have $e_{1}=1$ and $e_{q c}=z e_{c}$. Then, from the Jordan decomposition $C=C_{s} C_{u}$, where $C_{s}=P \operatorname{Diag}\left(c_{1}, \ldots, c_{n}\right) P^{-1}$, one draws $e_{C_{u}}:=C_{u}^{l_{q}}$ and $e_{C_{s}}:=P \operatorname{Diag}\left(e_{c_{1}}, \ldots, e_{c_{n}}\right) P^{-1}$. Last, $e_{C}:=e_{C_{s}} e_{C_{u}}$. Thus $\sigma_{q} X=A X$ admits the following non canonical fundamental solutions:

$$
\mathcal{X}^{(0)}:=M^{(0)} e_{A^{(0)}} \text { and } \mathcal{X}^{(\infty)}:=M^{(\infty)} e_{A^{(\infty)}} .
$$

\footnotetext{
${ }^{(19)}$ All definitions and constructions given at 0 can be applied at $\infty$ by using the coordinate $w:=1 / z$.

${ }^{(20)}$ The conventions used to obtain a matrix (and not a quadritensor) as the result of tensoring two matrices are detailed in loc. cit..
} 
The Birkhoff connection matrix is then defined as:

$$
P:=\left(\mathcal{X}^{(\infty)}\right)^{-1} \mathcal{X}^{(0)} \in \mathrm{GL}_{n}\left(\mathcal{M}\left(\mathbf{E}_{q}\right)\right) .
$$

In order to give it a functorial and even galoisian meaning, we record two basic facts. First [30, lemma 1.2.4.1, p. 935], if $F^{(0)}$ is a meromorphic (at 0) morphism from $A^{(0)}$ to $B^{(0)}$, then of course $F^{(0)} e_{A^{(0)}}=e_{B^{(0)}} R^{(0)}$ where $R^{(0)}$ is elliptic. But more is true: from the special form of our solutions, one can deduce that $R^{(0)} \in \operatorname{Mat}_{p, n}(\mathbf{C})$. (Similarly at $\infty$.) This is used in the context of the following commutative diagram:

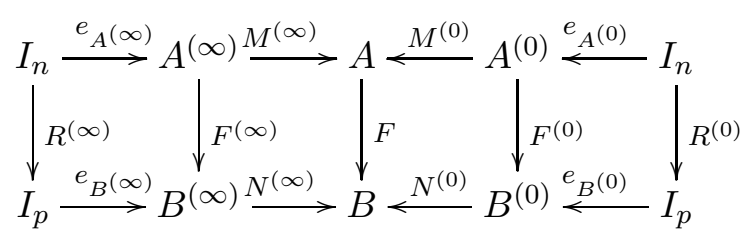

One can start from $F$ and complete it outwards, or start from $R^{(0)}$ and $R^{(\infty)}$ and complete it inwards.

As for the tensor properties, the basic fact is that it is impossible to choose the family of functions $e_{c}$ so that $e_{c} e_{d}=e_{c d}$. Thus we are led to introduce the cocycle of elliptic functions $\phi(c, d):=\frac{e_{c} e_{d}}{e_{c d}}$ and to extend it to matrices (through their eigenvalues) so as to have the formula:

$$
e_{C_{1}} \otimes e_{C_{2}}=e_{C_{1} \otimes C_{2}} \Phi\left(C_{1}, C_{2}\right) .
$$

Note that for unipotent matrices there is no twisting since $e_{1}=1$.

4.1.0.1. The tensor category $\mathcal{C}_{f}$ of connection data. - Its objects are triples $\left(A^{(0)}, P, A^{(\infty)}\right)$, where $A^{(0)}, A^{(\infty)} \in \mathrm{GL}_{n}(\mathbf{C})$ and $P \in \mathrm{GL}_{n}\left(\mathcal{M}\left(\mathbf{E}_{q}\right)\right)$. Morphisms from $\left(A^{(0)}, P, A^{(\infty)}\right)$ to $\left(B^{(0)}, Q, B^{(\infty)}\right)$ are pairs $\left(R^{(0)}, R^{(\infty)}\right) \in$ $\operatorname{Mat}_{p, n}(\mathbf{C})^{2}$ such that:

$$
\begin{gathered}
R^{(\infty)} P=Q R^{(0)}, \\
F^{(0)}:=e_{B^{(0)}} R^{(0)}\left(e_{A^{(0)}}\right)^{-1} \text { is meromorphic at } 0 \\
F^{(\infty)}:=e_{B^{(\infty)}} R^{(\infty)}\left(e_{A^{(\infty)}}\right)^{-1} \text { is meromorphic at } \infty .
\end{gathered}
$$

In loc. cit. an explicit condition is given ensuring these meromorphies, but we shall not need it. (It is used to guarantee that the following constructions do work.) 
Now the tensor product has to be twisted in order to get the theorem we need. For morphisms, and for the left and right components of objects, we use the usual tensor product. For the middle component, we shall use the twisted tensor product, defined as follows:

$$
\left(A_{1}^{(0)}, P_{1}, A_{1}^{(\infty)}\right) \otimes\left(A_{2}^{(0)}, P_{2}, A_{2}^{(\infty)}\right):=\left(A_{1}^{(0)} \otimes A_{2}^{(0)}, P_{1} \otimes P_{2}, A_{1}^{(\infty)} \otimes A_{2}^{(\infty)}\right),
$$

where:

$$
P_{1} \otimes P_{2}:=\Phi\left(A_{1}^{(\infty)}, A_{2}^{(\infty)}\right)\left(P_{1} \otimes P_{2}\right)\left(\Phi\left(A_{1}^{(0)}, A_{2}^{(0)}\right)\right)^{-1} .
$$

Theorem 4.1. - The tensor categories $\mathcal{E}_{f}$ and $\mathcal{C}_{f}$ are equivalent.

Proof. - Because of the non canonical choice, one does not define a functor from one of these categories to the other. Instead, one defines yet another category $\mathcal{S}_{f}$ with objects $\left(A^{(0)}, M^{(0)}, A^{(\infty)}, M^{(\infty)}\right)$ and with morphisms $\left(R^{(0)}, R^{(\infty)}\right)$, all being subject to adequate conditions. The tensor structure on $\mathcal{S}_{f}$ is the natural one. Then functors from $\mathcal{S}_{f}$ to $\mathcal{E}_{f}$ and $\mathcal{C}_{f}$ are easily defined. Note that the proof of the essential surjectivity of the second functor is essentially due to Birkhoff (it rests on his theorem of factorisation of analytic matrices).

4.1.0.2. The Galois group of $\mathcal{E}_{f}$ and $\mathcal{C}_{f}$. - From the description of $\mathcal{C}_{f}$, it is clear how to define fiber functors $\omega_{f}^{(0)}$ and $\omega_{f}^{(\infty)}$ on it. These extend to the local categories obtained by keeping only the 0 or $\infty$ component, and by allowing meromorphic morphisms. One thus obtains the local Galois groups $G_{f}^{(0)}$ and $G_{f}^{(\infty)}$ that were described in section 2.2. We want to use $P$ to connect them. More precisely, we should like each value $P(a) \in \mathrm{GL}_{n}(\mathbf{C})$ to behave like a "connection formula" in Riemann-Hilbert correspondance, and so be a galoisian isomorphism from $\omega^{(0)}(A)$ to $\omega^{(\infty)}(A)$. This does not work because the formation of the Birkhoff matrix is not $\otimes$-compatible: that is, $\left(P_{1} \otimes P_{2}\right)(a) \neq P_{1}(a) \otimes P_{2}(a)$. We shall therefore twist $P$ in order to obtain tensor-compatibility and also functoriality. This is done as follows.

One can define explicitly a family of (abstract) group morphisms $g_{a}$ from $\mathbf{C}^{*}$ to itself such that $g_{a}(q)=a$ for all $a \in \mathbf{C}^{*}$. Then we set $\psi_{a}(c):=\frac{e_{c}(a)}{g_{a}(c)}$ and we extend each function $\psi_{a}$ to a function $\Psi_{a}$ on matrices, through their eigenvalues. Last, we define:

$$
\check{P}(a):=\left(\Psi_{a}\left(A^{(\infty)}\right)^{-1} P(a) \Psi_{a}\left(A^{(0)}\right),\right.
$$

\footnotetext{
$\overline{{ }^{(21)} \text { In }[30,3.2 .2 .2]}$, the stated condition is $g_{a}(q)=1$, but it is a typographical error.
} 
and can prove that, for each $a \in \mathbf{C}^{*}$, one has an isomorphism of fiber functors $\left(A^{(0)}, P, A^{(\infty)}\right) \leadsto \check{P}(a)$ from $\omega_{f}^{(0)}$ to $\omega_{f}^{(\infty)}$. Since $\check{P}(a)$ is not defined for all $a$, this actually applies to a smaller category than $\mathcal{E}_{f}$, but any given object belongs to "most" of these subcategories.

Theorem 4.2. - The group generated by $G_{f}^{(0)}$, one particular conjugate $(\check{P}(a))^{-1} G_{f}^{(\infty)} \check{P}(a)$ and the set of all defined values $(\check{P}(b))^{-1} \check{P}(a)$ is Zariskidense in the global Galois group of $A$.

The proof uses Chevalley criterion: any line in any tensor construction that is fixed by the smaller group is fixed by the bigger one. It rests on the following useful fact: if $x$ is an eigenvector for $G_{f}^{(0)}$, then it is an eigenvector for $\Psi_{a}\left(A^{(0)}\right)$. We shall sketch the proof in our case of interest in the next subsection.

4.2. The global Galois group with integral slopes. - We now extend the results above to the case of irregular equations with integral slopes. As the extension involves no new idea, our presentation will be concise. The category $\mathcal{E}_{1}$ of interest has as objects systems with matrix $A \in \mathrm{GL}_{n}(\mathbf{C}(z))$ such that their slopes at 0 and at $\infty$ are integral; and as morphisms $A \rightarrow B$ matrices $F \in \operatorname{Mat}_{p, n}(\mathbf{C}(z))$ such that $\left(\sigma_{q} F\right) A=B F$. The tensor product is the natural one and makes it a neutral tannakian category. Each object $A$ of $\mathcal{E}_{1}$ can be written, non canonically:

$$
A=M^{(0)}\left[A^{(0)}\right]=M^{(\infty)}\left[A^{(\infty)}\right],
$$

where $M^{(0)} \in \mathrm{GL}_{n}(\mathbf{C}(\{z\})), M^{(\infty)} \in \mathrm{GL}_{n}(\mathbf{C}(\{w\}))$ and $A^{(0)}, A^{(\infty)}$ are in Birkhoff-Guenther normal form.

To define solutions, we choose once and for all a function $\theta$ such that $\sigma_{q} \theta=z \theta$ and an arbitrary direction of summation in $\mathbf{E}_{q}$. Because of this, the following constructions are only valid on a subcategory of $\mathcal{E}_{1}$, but each particular object of $\mathcal{E}_{1}$ belongs to "most" of these subcategories. We shall call $A_{p}^{(0)}, A_{p}^{(\infty)}$ the pure systems associated to $A^{(0)}, A^{(\infty)}$ by the gr functor (hence there block-diagonal parts). Let $S^{(0)}$ the meromorphic isomorphism from $A_{p}^{(0)}$ to $A^{(0)}$ obtained by summation along the selected direction mentioned above; and similarly at infinity. Then, calling $\mu_{1}, \ldots, \mu_{k}$ the slopes of $A^{(0)}$ and $r_{1}, \ldots, r_{k}$ their multiplicities, let $\Gamma^{(0)}:=\operatorname{Diag}\left(\theta^{\mu_{1}} I_{r_{1}}, \ldots, \theta^{\mu_{k}} I_{r_{k}}\right)$. We have $A_{p}^{(0)}=\Gamma^{(0)}\left[A_{f}^{(0)}\right]$ with $A_{f}^{(0)} \in \mathrm{GL}_{n}(\mathbf{C})$. In the end, using the similar 
notations at infinity, we put:

$$
e_{A^{(0)}}:=S^{(0)} \Gamma^{(0)} e_{A_{f}^{(0)}} \text { and } e_{A^{(\infty)}}:=S^{(\infty)} \Gamma^{(\infty)} e_{A_{f}^{(\infty)}} .
$$

Thus $\sigma_{q} X=A X$ admits the following non canonical fundamental solutions:

$$
\mathcal{X}^{(0)}:=M^{(0)} e_{A^{(0)}} \text { and } \mathcal{X}^{(\infty)}:=M^{(\infty)} e_{A^{(\infty)}} .
$$

The Birkhoff connection matrix is then defined as:

$$
P:=\left(\mathcal{X}^{(\infty)}\right)^{-1} \mathcal{X}^{(0)} \in \mathrm{GL}_{n}\left(\mathcal{M}\left(\mathbf{E}_{q}\right)\right) .
$$

Its tensor behaviour is exactly similar to that observed in the fuchsian case and we shall set, in appropriate context:

$$
\left(A_{1}^{(0)}, P_{1}, A_{1}^{(\infty)}\right) \otimes\left(A_{2}^{(0)}, P_{2}, A_{2}^{(\infty)}\right):=\left(A_{1}^{(0)} \otimes A_{2}^{(0)}, P_{1} \otimes P_{2}, A_{1}^{(\infty)} \otimes A_{2}^{(\infty)}\right),
$$

where:

$$
P_{1} \underline{\otimes} P_{2}:=\Phi\left(\left(A_{1}\right)_{f}^{(\infty)},\left(A_{2}\right)_{f}^{(\infty)}\right)\left(P_{1} \otimes P_{2}\right)\left(\Phi\left(\left(A_{1}\right)_{f}^{(0)},\left(A_{2}\right)_{f}^{(0)}\right)\right)^{-1} .
$$

The functorial behaviour requires some more comments. Let $B$ an object of rank $p$ in $\mathcal{E}_{1}$ and $B^{(0)}, N^{(0)}, B_{p}^{(0)}, T^{(0)}, B_{f}^{(0)}, \Delta^{(0)}, \mathcal{Y}^{(0)}, \mathcal{Y}^{(\infty)}$, and $Q$ the associated data corresponding respectively to $A^{(0)}, M^{(0)}, A_{p}^{(0)}, S^{(0)}, A_{f}^{(0)}$, $\Gamma^{(0)}, \mathcal{X}^{(0)}, \mathcal{X}^{(\infty)}$ and $P$. Let $F$ be a morphism from $A$ to $B$. Then we have a commutative diagram:

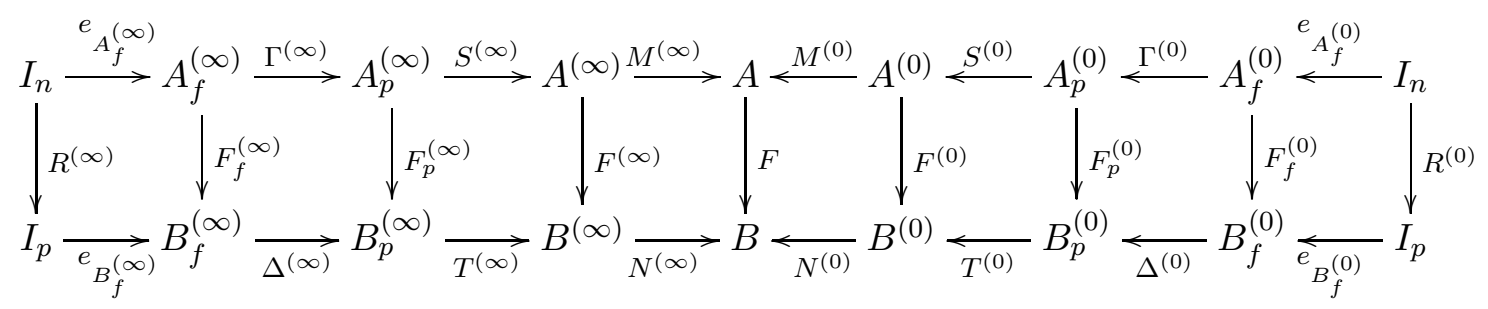

Of course, all vertical arrows can be defined from $F$. For instance, $F^{(0)}:=\left(N^{(0)}\right)^{-1} \circ F \circ M^{(0)} \in \operatorname{Mat}_{p, n}(\mathbf{C}(\{z\}))$ is a morphism in $\mathcal{E}_{1}^{(0)}$, and similarly at $\infty$. Then one can see that $F_{p}^{(0)}:=\left(T^{(0)}\right)^{-1} \circ F^{(0)} \circ S^{(0)}$ is actually $\operatorname{gr} F^{(0)}$ (and similarly at $\infty$ ); and, from the block-diagonal structures of the involved matrices, one can see that $F_{f}^{(0)}:=\left(\Delta^{(0)}\right)^{-1} \circ F_{p}^{(0)} \circ \Gamma^{(0)}$ is actually equal to $F_{p}^{(0)}=\operatorname{gr} F^{(0)}$, the block-diagonal of $F^{(0)}$. Then, from the lemma already quoted [30, lemma 1.2.4.1, p. 935], we see that $R^{(0)}:=\left(e_{B_{f}^{(0)}}\right)^{-1} \circ F_{f}^{(0)} \circ e_{A_{f}^{(0)}} \in \operatorname{Mat}_{p, n}(\mathbf{C})$ and similarly at $\infty$.

Conversely, if we are given the two lines and the most external vertical arrows $R^{(0)}, R^{(\infty)}$, the condition to be able to go inwards and fill in the other 
vertical arrows to get a commutative diagram is that $Q R^{(0)}=R^{(\infty)} P$. The condition to get a rational $F$ is that $F^{(0)} \in \operatorname{Mat}_{p, n}(\mathbf{C}(\{z\}))$ and similarly at $\infty$. Indeed, from the functional equation $\sigma_{q} F^{(0)}=B^{(0)} F^{(0)}\left(A^{(0)}\right)^{-1}$ and the fact that $A^{(0)}, B^{(0)}$ are in Birkhoff-Guenther normal form, one deduces that $F^{(0)}$ is meromorphic on $\mathbf{C}$, and similarly at $\infty$, so that $F$ is actually meromorphic on the Riemann sphere, thus rational.

4.2.0.3. The tensor category $\mathcal{C}_{1}$ of connection data. - Its objects are triples $\left(A^{(0)}, P, A^{(\infty)}\right)$, where $A^{(0)}, A^{(\infty)} \in \mathrm{GL}_{n}(\mathbf{C}(\{z\}))$ are in Birkhoff-Guenther normal form and $P \in \mathrm{GL}_{n}\left(\mathcal{M}\left(\mathbf{E}_{q}\right)\right)$. Morphisms from $\left(A^{(0)}, P, A^{(\infty)}\right)$ to $\left(B^{(0)}, Q, B^{(\infty)}\right)$ are pairs $\left(R^{(0)}, R^{(\infty)}\right) \in \operatorname{Mat}_{p, n}(\mathbf{C})^{2}$ such that:

$$
\begin{gathered}
R^{(\infty)} P=Q R^{(0)}, \\
F^{(0)}:=e_{B^{(0)}} R^{(0)}\left(e_{A^{(0)}}\right)^{-1} \text { is meromorphic at } 0 \\
F^{(\infty)}:=e_{B^{(\infty)}} R^{(\infty)}\left(e_{A^{(\infty)}}\right)^{-1} \text { is meromorphic at } \infty .
\end{gathered}
$$

Remark 4.3. - We saw in 4.1.0.1 that there was an explicit condition (although we did not state it) on $R^{(0)}$ for $F_{p}^{(0)}=F_{f}^{(0)}$ to be meromorphic at 0 . Here, we must add a new condition to ensure that $F^{(0)}$ is also meromorphic at 0 . This condition is obviously related to the summations $S^{(0)}$ and $T^{(0)}$. We have not so far an explicit criterion, but it could be related to the way $F_{p}^{(0)}$ links the classifying cohomology class in $H^{1}\left(\mathbf{E}_{q}, \mathcal{F}_{A_{p}^{(0)}}\right)$ corresponding to $A^{(0)}$ to the classifying cohomology class in $H^{1}\left(\mathbf{E}_{q}, \mathcal{F}_{B_{p}^{(0)}}\right)$ corresponding to $B^{(0)}$.

The tensor structure is defined as follows. For morphisms, and for the left and right components of objects, we use the usual tensor product. For the middle component, we shall use the twisted tensor product, defined as follows:

$$
\left(A_{1}^{(0)}, P_{1}, A_{1}^{(\infty)}\right) \otimes\left(A_{2}^{(0)}, P_{2}, A_{2}^{(\infty)}\right):=\left(A_{1}^{(0)} \otimes A_{2}^{(0)}, P_{1} \otimes P_{2}, A_{1}^{(\infty)} \otimes A_{2}^{(\infty)}\right),
$$

where:

$$
P_{1} \underline{\otimes} P_{2}:=\Phi\left(A_{1}^{(\infty)}, A_{2}^{(\infty)}\right)\left(P_{1} \otimes P_{2}\right)\left(\Phi\left(A_{1}^{(0)}, A_{2}^{(0)}\right)\right)^{-1} .
$$

Recall that we have extended the definition of $\Phi$ to this setting.

Theorem 4.4. - The tensor categories $\mathcal{E}_{1}$ and $\mathcal{C}_{1}$ are equivalent.

Proof. - The method and the proof are the same as in the fuchsian case: we use an enriched category $\mathcal{S}_{1}$ with objects $\left(A^{(0)}, M^{(0)}, A^{(\infty)}, M^{(\infty)}\right)$ and with morphisms $\left(R^{(0)}, R^{(\infty)}\right)$, all being subject to obvious conditions. The tensor structure on $\mathcal{S}_{1}$ is the natural one. Then functors from $\mathcal{S}_{1}$ to $\mathcal{E}_{1}$ and $\mathcal{C}_{1}$ are defined and proved to be $\otimes$-equivalences exactly as in the fuchsian case. 
4.2.0.4. The Galois group of $\mathcal{E}_{1}$ and $\mathcal{C}_{1}$. - From the description of $\mathcal{C}_{1}$, it is clear how to define fiber functors $\omega_{1}^{(0)}$ and $\omega_{1}^{(\infty)}$ on it and that their extension to the local categories $\mathcal{E}_{1}^{(0)}$ and $\mathcal{E}_{1}^{(\infty)}$ yields local Galois groups which are precisely the Galois group $G_{1}^{(0)}$ studied in this paper and its counterpart $G_{1}^{(\infty)}$ at $\infty$.

Also the formula:

$$
\check{P}(a):=\left(\Psi_{a}\left(A^{(\infty)}\right)^{-1} P(a) \Psi_{a}\left(A^{(0)}\right),\right.
$$

extends here with the only adaptation that $\Psi_{a}\left(A^{(0)}\right)$ means $\Psi_{a}\left(A_{f}^{(0)}\right)$, and similarly at $\infty$. Again, one finds that, for each $a \in \mathbf{C}^{*}$, one has an isomorphism of fiber functors $\left(A^{(0)}, P, A^{(\infty)}\right) \leadsto \check{P}(a)$ from $\omega_{1}^{(0)}$ to $\omega_{1}^{(\infty)}$ (again, on appropriate subcategories).

Theorem 4.5. - The group generated by $G_{1}^{(0)}$, one particular conjugate $(\check{P}(a))^{-1} G_{1}^{(\infty)} \check{P}(a)$ and the set of all defined values $(\check{P}(b))^{-1} \check{P}(a)$ is Zariskidense in the global Galois group of $A$.

Proof. - The proof uses again Chevalley criterion in a similar way to loc. cit.. Suppose we have two lines $D^{(0)}$ and $D^{(\infty)}$ that are respectively fixed by $G_{1}^{(0)}$ and $G_{1}^{(\infty)}$ and such that each $\check{P}(a)$ sends $D^{(0)}$ to $D^{(\infty)}$. Taking generators $x^{(0)}, x^{(\infty)}$, we se by tannakian duality that they define rank one subobjects $x^{(0)}: a^{(0)} \rightarrow A^{(0)}$ and $x^{(\infty)}: a^{(\infty)} \rightarrow A^{(\infty)}$. By the lemma quoted at the end of the previous subsection, the fact that $x^{(0)}, x^{(\infty)}$ are respectively eigenvectors of $G_{1}^{(0)}, G_{1}^{(\infty)}$ implies that the value $P(a)$ of the non twisted connection matrix sends $D^{(0)}$ to $D^{(\infty)}$, so that $P(a) x^{(0)}=p(a) x^{(\infty)}$ for some $p(a) \in \mathbf{C}$. But then $p$ is a non trivial elliptic function, $\left(a^{(0)}, p, a^{(\infty)}\right)$ is a rank one object of $\mathcal{C}_{1}$ and $\left(x^{(0)}, x^{(\infty)}\right)$ an embedding of this object as a subobject of $\left(A^{(0)}, P, A^{(\infty)}\right)$. Then, by functoriality, all elements of the global Galois group must fix this subobject, whence the two lines.

Corollary 4.6. - Topological generators of the Stokes Lie algebra at 0 and $\infty$ together with topological generators of the local pure Galois groups and the values of $\check{P}(a)$ are together topological generators of the global Galois group.

\section{The inverse problem}


5.1. Known results. - To our knowledge there existed before almost no result on the local inverse problem that we shall solve below (for the integral slope case). We will review the known results on the global inverse problem.

As far as we know, the first significant result on the global inverse problem of the $q$-difference Galois theory is due to P. Etingof [10] (Proposition 3.4, page 7$)$.

Proposition 5.1. - Let $G$ be any connected complex linear algebraic group, there exists $\rho>0$ (depending on $G$ ) such that, for all $0<|q|<\delta$, there exists a rational regular difference system $\sigma_{q} Y=A Y$ whose q-difference Galois group is $G$.

We recall that the system $\sigma_{q} Y=A Y$ is regular if $A(0)=A(\infty)=I$.

The proof uses the following result ( $c f$. [34]).

Lemma 5.2. - Let $G$ be any complex linear algebraic goup, then there exists $g_{1}, \ldots, g_{m} \in G$ such that the subgroup generated by $g_{1}, \ldots, g_{m}$ is Zariski dense in $G$.

We recall that the Tretkoffs used this lemma (and the Riemann-Hilbert correspondance) to solve the inverse problem of the Galois differential theory with regular singular systems.

If $G$ is abelian, it is possible to improve the proposition 5.1

Proposition 5.3. - Let $G$ be any abelian connected complex linear algebraic group, then, for all $q \in \mathbf{C}^{*},|q| \neq 1$, there exists a rational regular difference system $\sigma_{q} Y=A Y$ whose q-difference Galois group is $G$.

This proposition follows from [30], using the following lemma.

Lemma 5.4. - Let $G$ be any abelian connected complex linear algebraic goup, then there exists a rational dominant map $f: \mathbf{E}_{q} \rightarrow G$.

Here $\mathbf{E}_{q}$ is seen as a projective algebraic curve (an elliptic curve). The proof of this lemma follows from the existence of an isomorphism $G \approx\left(G_{m}\right)^{k} \times\left(G_{u}\right)^{l}$.

From propositions 5.1 and 5.3 we can conjecture that for every connected complex linear algebraic group $G$ and for all $q \in \mathbf{C}^{*},|q| \neq 1$, there exists a rational regular difference system $\sigma_{q} Y=A Y$ whose $q$-difference Galois group is $G$. 
Such a system will have in general "a lot of singularities". Below we will attack the global inverse problem in the opposite direction, searching a system with a minimal number of singularities in the spirit of a $q$-analog of the Abhyankar conjecture.

Another source of solutions of the inverse problem are of course the known solutions of the direct problem, in particular from the computation of the $q$ difference Galois groups of the generalized q-hypergeometric equations (regularsingular or not). One can find a complete solution of this last problem in a series of papers of J. Roques [26, 27, 25. Limiting ourselves to the cases of simple groups, the complete list obtained by $\mathrm{J}$. Roques is: $\mathrm{SL}(n, \mathbf{C}), \mathrm{SO}(n, \mathbf{C})$, $\operatorname{Sp}(2 n, \mathbf{C})$.

It is interesting to compare with the differential case ( $c f .[\mathbf{2}], \mathbf{9}], \mathbf{1 3},[\mathbf{1 8}])$. The simple groups which are differential groups of generalized hypergeometric differential equations (regular-singular or not) are: $\mathrm{SL}(n, \mathbf{C}), \operatorname{SO}(n, \mathbf{C})$, $\operatorname{Sp}(2 n, \mathbf{C})$ and $\ldots$ the group $G_{2}$ ! Therefore the only difference between the $q$-difference case and the differential case is the exceptional group $G_{2}$.

\subsection{Linear algebraic groups: reminders and complements. -}

5.2.1. Notations and definitions. Levi decomposition. - In the following all the algebraic groups are complex linear algebraic groups. In general $G$ is a linear algebraic group, $\mathfrak{g}$ is its Lie algebra, $T \subset G$ is a torus, $\mathfrak{t}$ the Lie algebra of $T$, and $D \subset G$ is an abelian semi-simple group.

An algebraic group $\mathrm{G}$ contains a unique maximal normal solvable subgroup, this subgroup is closed. Its identity component is called the radical $R(G)$ of $G$.

We will denote $R_{u}(G)$ the unipotent radical of $G$ (i.e. the set of unipotent elements of $R(G)$ ). A group $G$ is reductive if and only if $R_{u}(G)=\{e\}$.

Definition 5.5. - A Levi subgroup of a linear algebraic group $G$ is a maximal reductive subgroup.

We have an exact sequence:

$$
\{e\} \rightarrow R_{u}(G) \rightarrow G \rightarrow G / R_{u}(G) \rightarrow\{e\}
$$

and, if $H \subset G$ is a Levi subgroup, then the quotient map $G \rightarrow G / R_{u}(G)$ induces an isomorphism $H \rightarrow G / R_{u}(G)$. More precisely we have the following result (essentially due to Mostow).

Proposition 5.6. - Let $G$ be a linear algebraic group. 
(i) If $H \subset G$ is a Levi subgroup, then $G$ is a semi-direct product: $G=$ $R_{u}(G) \rtimes H$.

(ii) Any two Levi subgroups of $G$ are conjugate under an inner automorphism.

(iii) If $H \subset G$ is a subgroup and if the quotient map $G \rightarrow G / R_{u}(G)$ induces an isomorphism $H \rightarrow G / R_{u}(G)$, then $H$ is a Levi subgroup.

Proof. - For (i) and (ii) cf. [19] (a subgroup is fully reducible if and only if it is reductive).

Let $H \subset G$ be a subgroup such that the quotient map $G \rightarrow G / R_{u}(G)$ induces an isomorphism $H \rightarrow G / R_{u}(G), H$ is reductive, therefore it is contained in a maximal reductive subgroup $H^{\prime}$ and $H=H^{\prime}$.

A Levi decomposition of a linear algebraic group $G$ is an isomorphism $G \approx$ $U \rtimes S$, where $S$ is reductive and $U$ is unipotent.

5.2.2. Diagonalisable and triangularisable groups. - We shall recall the notions of diagonalisable and of triangularizable algebraic group. The properties of the diagonalisable groups and of the triangularisable connected groups are well known, but for the triangularisable non connected groups we do not know good references hence, for sake of completeness, we shall detail the necessary results.

An algebraic group $G$ is diagonalizable if and only if it is abelian and semisimple $\left(G=G_{s}\right)$. If $G$ is diagonalizable, then every representation of $G$ is diagonalizable in the matrix sense. An algebraic group $G$ is diagonalisable if and only if there exists a faithful representation of $G$ which is diagonalisable in the matrix sense.

We will say that a linear algebraic group $G$ is triangularizable if there exists a faithful triangular representation. A triangularizable group is solvable.

A solvable connected linear algebraic group is triangularizable (Lie-Kolchin theorem). In particular a unipotent group is triangularizable.

Proposition 5.7. - A linear algebraic group is triangularizable if and only if $G \approx U \rtimes D$, where $U$ is unipotent and $D$ is abelian and semi-simple. Then $U=R_{u}(G)$ and $D \approx G / R_{u}(G)$.

The Levi subgroups of a triangularizable algebraic group $G$ are the maximal abelian semi-simple subgroups. If $G$ is connected, the Levi subgroups are the maximal tori.

Proof. - 1. If $G \approx U \rtimes D$, where $U$ is unipotent and $D$ is abelian and semi-simple, $G$ is triangularizable by [14, I.7, lemma, p 20]. 
2. We suppose that $G$ is triangularizable, there exists a faithful representation $\rho: G \rightarrow \mathrm{GL}_{n}(\mathbf{C})$, such that $\rho(G)$ is an upper triangular subgroup of $\mathrm{GL}_{n}(\mathbf{C})$, a subgroup of the upper triangular subgroup $T_{n}$. We denote $U_{n}$ (resp. $D_{n}$ ) the unipotent upper-triangular subgroup (resp. the diagonal subgroup) of $\mathrm{GL}_{n}(\mathbf{C})$, then $T_{n}=U_{n} \rtimes D_{n}, T_{n} / U_{n}=D_{n}$.

There exists a Levi decomposition $G=U \rtimes D$, where $U$ is unipotent and $D$ reductive. Then $\rho(U)$ is unipotent, therefore it is a subgroup of $U_{n}$ and $\rho$ induces an injective morphism $D=G / U \rightarrow T_{n} / U_{n}=D_{n}$. The group $D_{n}$ is abelian semi-simple, and $D$ is isomorphic to a subgroup, therefore $D$ is abelian semi-simple.

3. The Levi subgroups are abelian semi-simple and any abelian semi-simple subgroup is reductive, the result follows.

\section{3. $\Theta$-structures on linear algebraic groups. -}

\subsubsection{Weights and coweights. -}

Definition 5.8. - Let $G$ be an abelian semi-simple group. The weight group $G^{\bullet}$ of $G$ is the group of homomorphisms of algebraic groups $G \rightarrow \mathbf{C}^{*}$.

A weight on $G$ is also called a character on $G$.

The group $G^{\bullet}$ is an abelian finitely generated group.

The weight functor $G \leadsto G^{\bullet}$ is an antiequivalence of categories between abelian semi-simple algebraic groups and finitely generated abelian groups. The quasi inverse of the weight functor is $\operatorname{Hom}_{g r}\left(., \mathbf{C}^{*}\right)$.

Let $f: \mathbf{C}^{*} \rightarrow \mathbf{C}^{*}$ be a homomorphism of algebraic groups, then $f: z \mapsto z^{n}$, $n \in \mathbf{Z}$ is the degree of $f$ and we denote $\operatorname{deg} f=n$.

Definition 5.9. - Let $D$ be an abelian semi-simple group. A coweight on $D$ is a homomorphism of algebraic groups $\mathbf{C}^{*} \rightarrow D$.

A coweight on $D$ is also called a one parameter subgroup of $D$.

If $\chi: \mathbf{C}^{*} \rightarrow D$ is a coweight on $D$, its image is contained in the maximal torus $T \subset D$, therefore it is also a coweight on $T$.

For every weight $\xi$ and every coweight $\chi$ on an algebraic abelian semi-simple group $D$, we set

$$
<\xi, \chi>:=\operatorname{deg}(\xi \circ \chi) .
$$


Definition 5.10. - Let $T$ be a complex algebraic torus. The weight lattice $T^{\bullet}$ of $T$ is the group of weights $T \rightarrow \mathbf{C}^{*}$, and the coweight lattice $T_{\bullet}$ of $T$ is the group of coweights $\mathbf{C}^{*} \rightarrow T$.

The groups $T^{\bullet}$ and $T_{\bullet}$ are both free abelian groups whose rank is the dimension of $T$. The map $(\xi, \chi) \mapsto<\xi, \chi>:=\operatorname{deg}(\xi \circ \chi)$ is a canonical non degenerate pairing $T^{\bullet} \times T_{\bullet} \rightarrow \mathbf{Z}$ (cf. for example [12, 16.1]).

The weight functor $T \leadsto T^{\bullet}$, resp. the coweight functor $T \leadsto T_{\bullet}$ is an antiequivalence, resp. an equivalence of categories between algebraic tori and finitely generated free abelian groups. The quasi inverse of the weight functor is $\operatorname{Hom}_{g r}\left(. \mathbf{C}^{*}\right)$.

An isomorphism of algebraic torus $\Phi:\left(\mathbf{C}^{*}\right)^{\mu} \rightarrow T$ gives a $\mathbf{Z}$-basis of $T_{\bullet}$ and the inverse isomorphism $\Phi^{-1}: T \rightarrow\left(\mathbf{C}^{*}\right)^{\mu}$ gives a $\mathbf{Z}$-basis of $T^{\bullet}$.

To a weight $\xi: T \rightarrow \mathbf{C}^{*}$ we associate its infinitesimal counterpart $L \xi: \mathfrak{t} \rightarrow \mathbf{C}$ (remember $\mathfrak{t}$ denotes the Lie algebra of $T$ ). We will sometimes "identify" the group of weights and the group of infinitesimal weights and we will interpret $T^{\bullet}$ as a $\mathbf{Z}$-submodule of the complex dual space $\mathfrak{t}^{*}$ of $\mathfrak{t}$. According to the tradition, we will freely use the additive notation for the weights. We will denote $\mathcal{W}_{\mathbf{R}}$ the real vector space $\mathbf{R} \otimes \mathbf{z} T^{\bullet}$.

For $\xi \in T^{\bullet}, \chi \in T_{\bullet}$, we define:

$$
<L \xi, L \chi>:=L \xi \circ L \chi(1)=<\xi, \chi>.
$$

Let $G$ be a linear algebraic group and let be $D$ an abelian semi-simple group. We recall that the roots of $D$ are the non trivial weights on $D$ for the adjoint action of $D$ on the Lie algebra $\mathfrak{g}$. We denote $\mathfrak{g}_{\xi}$ the root space associated to the root $\xi$ :

$$
\mathfrak{g}_{\xi}:=\{x \in \mathfrak{g} \mid \forall \lambda \in D,(\operatorname{Ad} \lambda)(x)=\xi(\lambda) x\} .
$$

We have $\mathfrak{g}=\mathfrak{g}_{0} \oplus \bigoplus_{\xi \in \mathcal{R}} \mathfrak{g}_{\xi}$, the sum being on the set of roots $\mathcal{R}$ and $\mathfrak{g}_{0}$ being the space of elements invariant by $D$.

If $D=T$ is connected (a torus), then

$$
\mathfrak{g}_{\xi}=\{x \in \mathfrak{g} \mid \forall \tau \in \mathfrak{t},(\operatorname{ad} \tau)(x)=L \xi(\tau) x\} .
$$

Let $\chi$ be a non trivial coweight on a torus $T \subset G$, it induces a grading of Lie algebras on $\mathfrak{g}$ :

$\forall k \in \mathbf{Z}, g r_{\chi}^{k}:=\left\{x \in \mathfrak{g} \mid \forall t \in \mathbf{C}^{*},(\operatorname{Ad} \chi(t))(x)=t^{k} x\right\}=\{x \in \mathfrak{g} \mid[L \chi(1), x]=k x\}$. 
If $\xi$ is a weight on $T$, then there exists a unique $k \in \mathbf{Z}$ such that $\mathfrak{g}_{\xi} \subset g r_{\chi}^{k}$ and we have $k=<\xi, \chi>$. In particular $\mathfrak{g}_{0} \subset g r_{\chi}^{0}$. We have:

$$
g r_{\chi}^{k}=\bigoplus_{<\xi, \chi>=k} \mathfrak{g}_{\xi} .
$$

5.3.2. $\Theta$-coweights and $\Theta$-structures. -

Definition 5.11. - Let $D \subset G$ be an abelian semi-simple subgroup and $\mathcal{P}:=\left\{\xi_{i}\right\}_{i \in I}$ a finite family of non trivial weights on $D$. We will say that a coweight $\chi$ on $D$ is positive (resp. negative) on $\mathcal{P}$ if, for every $i \in I$, $<\xi_{i}, \chi>>0\left(\right.$ resp. $\left.<\xi_{i}, \chi><0\right)$.

Definition 5.12. - Let $G$ be a triangularizable linear algebraic group. Let $D$ be a Levi subgroup of $G$. By proposition [5.7, $D$ is abelian semi-simple. A $\Theta$-coweight on $D$ is a coweight on $D$ which is negative on the family of roots for the adjoint action of $D$ on the Lie algebra $\mathfrak{g}$ of $G$.

If $\chi$ is a $\Theta$-coweight on $D$, then $\mathfrak{g}_{0}=g r_{\chi}^{0}$ and $\mathfrak{g}=\bigoplus_{k \in-\mathbf{N}} g r_{\chi}^{k}$.

\subsubsection{1. $\Theta$-structures. -}

Definition 5.13. - We will say that a linear algebraic group $G$ admits a $\Theta$-structure if it is triangularizable and if there exists a $\Theta$-coweight on a Levi subgroup of $G$.

Then, by conjugation, there exists $\Theta$-coweight on any Levi subgroup of $G$.

Definition 5.14. - Let $G$ be a linear algebraic group, let $D \subset G$ be an abelian semi-simple subgroup and $\chi$ a $\Theta$-coweight on $D$. We will say that $\chi$ is dominant if, for every root $\zeta$ on $D$, we have $\operatorname{dimg}_{\zeta} \leq-<\zeta, \chi>$.

Lemma 5.15. - Let $G$ be a triangularizable complex linear algebraic group, let $D \subset G$ be an abelian semi-simple subgroup. We suppose that $\chi$ is a $\Theta$ coweight on $D$. Then there exists a $\Theta$-coweight on $D$ which is dominant.

Proof. - Let $m \in \mathbf{N}^{*}$ and $\varphi_{m}: \mathbf{C}^{*} \rightarrow \mathbf{C}^{*}$ defined by $\varphi_{m}: t \mapsto t^{m}$. Then $\chi_{m}:=\chi \circ \varphi_{m}$ is a coweight on $T$, and for every root $\xi$, we have $<\xi, \chi><0$, that is $\left\langle\xi, \chi>\leq-1\right.$, whence $\left\langle\xi, \chi_{m}\right\rangle=m<\xi, \chi>\leq-m$, and $\chi_{m}$ is a $\Theta$-coweight. Then, for a sufficiently big $m\left(m \geq \max _{\xi \in \mathcal{R}}\left(\operatorname{dim} \mathfrak{g}_{\xi}\right)\right), \chi_{m}$ is dominant. 


\subsubsection{Existence of a $\Theta$-structure. -}

Remark 5.16. - $\quad$ 1. If $G=U \rtimes D$ is a Levi decomposition such that the semidirect product is not direct and if $D$ is an abelian finite group, then there exists no $\theta$-structure on $G$.

2. We suppose that there exists a $\Theta$-structure on a linear algebraic group $G$. If $\xi$ is a root, then $\xi^{-1}$ is not a root.

3. There exists a triangularizable connected linear algebraic group $G$ such that there exists no $\Theta$-structure on $G$. Let :

$$
G:=\left\{\left(\begin{array}{ccc}
1 & \alpha & \beta \\
0 & t & \gamma \\
0 & 0 & 1
\end{array}\right) \mid t \in \mathbf{C}^{*}, \alpha, \beta, \gamma \in \mathbf{C}\right\}
$$

it is triangular and it admits the infinitesimal roots 1 and -1 , therefore there exists no $\Theta$-structure on $G$.

A triangularizable group being given it seems difficult to find a practical criterium to decide if it admits a $\Theta$-structure. We shall give now a sufficient condition (we will use it below for the case of Borel subgroups of reductive groups).

Definition 5.17. - Let $G$ a linear algebraic group and $T \subset G$ a torus A good system of roots for the adjoint action of $T$ on $\mathfrak{g}$ is a set $\Sigma$ of roots such that

(i) $\Sigma$ is a $\mathbf{R}$-free subset of $W_{\mathbf{R}}$;

(ii) every root $\xi \in \mathcal{R}$ can be written $\xi=\sum_{k \in I} a_{i} \xi_{i}$, with, for every $i \in I$, $a_{i} \in \mathbf{R}_{+}$and $\xi_{i} \in \Sigma$.

Proposition 5.18. - Let $G$ a connected triangularisable group and $T \subset G$ a maximal torus. If there exists a good system of roots for the adjoint action of $T$ on $\mathfrak{g}$, then there exists a $\Theta$-structure on $G$.

Proof. - We prove firstly a preliminary lemma (part (ii) of this lemma will be used later).

Lemma 5.19. - (i) Let $f_{1}, \ldots, f_{\mu^{\prime}}$ independent $\mathbf{R}$-linear forms on $\mathbf{R}^{\mu}$, there exists $p=\left(p_{1}, \ldots, p_{\mu}\right) \in \mathbf{Z}^{\mu}$ such that $f_{i}(p)<0$ for all $i=1, \ldots, \mu^{\prime}$.

(ii) Let $f_{1}, \ldots, f_{\mu^{\prime}}$ non trivial $\mathbf{R}$-linear forms on $\mathbf{R}^{\mu}$, there exists $p=$ $\left(p_{1}, \ldots, p_{\mu}\right) \in \mathbf{Z}^{\mu}$ such that $f_{i}(p) \neq 0$ for all $i=1, \ldots, \mu^{\prime}$. 
Proof. - (i) The set $U:=\left\{y \in \mathbf{R}^{\mu} \mid f_{i}(y)<0, i=1, \ldots, \mu^{\prime}\right\}$ is a non void open subset of $\mathbf{R}^{\mu}$, therefore there exists $p^{\prime}=\left(p_{1}^{\prime}, \ldots, p_{\mu}^{\prime}\right) \in \mathbf{Q}^{\mu} \cap U$. If $y \in U$ and $a \in \mathbf{N}^{*}$, then $a y \in U$, the result follows.

(ii) The set $U:=\left\{y \in \mathbf{R}^{\mu} \mid f_{i}(y) \neq 0, i=1, \ldots, \mu^{\prime}\right\}$ is a non void open subset of $\mathbf{R}^{\mu}$, therefore there exists $p^{\prime}=\left(p_{1}^{\prime}, \ldots, p_{\mu}^{\prime}\right) \in \mathbf{Q}^{\mu} \cap U$. If $y \in U$ and $a \in \mathbf{N}^{*}$, then $a y \in U$, the result follows.

We can now prove the proposition.

Let $\Phi:\left(\mathbf{C}^{*}\right)^{\mu} \rightarrow T$ be an isomorphism of tori.

Let $\Sigma=\left\{\xi_{1}, \ldots, \xi_{\mu^{\prime}}\right\}$ be a good system of roots of $G$. For $i=1, \ldots, \mu^{\prime}$, we set $f_{i}:=L \xi_{i} \circ L \Phi$. We interpret $f_{1}, \ldots, f_{\mu^{\prime}}$ as linear forms on $\mathbf{R}^{\mu}$, by hypothesis they are independent, therefore we can apply the lemma 5.19 above. There exists $p=\left(p_{1}, \ldots, p_{\mu}\right) \in \mathbf{Z}^{\mu}$ such that $f_{i}(p)<0$ for all $i=1, \ldots, \mu^{\prime}$. We define a morphism $\chi: \mathbf{C}^{*} \rightarrow T$ by $\Phi^{-1} \circ \chi: t \rightarrow\left(t_{1}:=t^{p_{1}}, \ldots, t_{\mu}:=t^{p_{\mu}}\right)$, for $i=1, \ldots, \mu^{\prime}$, then we set $v_{i}:=f_{i} \circ L\left(\Phi^{-1} \circ \chi\right):=L \xi_{i} \circ L \chi$. We have $v_{i}(1)=f_{i}(p)<0$. If $\xi$ is a root, then $<L \xi, L \chi>=\sum_{i=1, \ldots, \mu^{\prime}} a_{i} v_{i}(1)$ with $a_{i} \geq 0, a_{1}+\cdots+a_{\mu^{\prime}}>0$ and therefore $<L \xi, L \chi>=<\xi, \chi><0$.

In the following proposition, $T$ is a maximal torus of $G$. One implication is proposition 5.18

Proposition 5.20. - If the dimension of $T$ is one, then $G$ admits admits a $\Theta$-structure if and only if there exists a good system of roots.

Proof. - If $G$ admits admits a $\Theta$-structure, then there exists a surjective morphism $\eta: \mathbf{C}^{*} \rightarrow T$ such that, for every root $\xi,\langle\xi, \eta\rangle$ is negative. Let $\xi_{1}$ be a root, then, for every root $\xi$, we have $L \xi=a L \xi_{1}$ with $a>0$, therefore $\left\{\xi_{1}\right\}$ is a good system of roots.

For basic definitions on Borel subgroups, positive systems of roots... $c f$.[12].

Proposition 5.21. - If $G^{+}$is a Borel subgroup of a connected reductive algebraic group, then there exists a $\Theta$-structure on $G^{+}$.

Proof. - Let $G^{+}$be a Borel subgroup of the connected reductive algebraic group $G$. Let $T$ be a maximal torus of $G$ contained in $G^{+}$, then $G^{+}$corresponds to a positive system of roots $\mathcal{R}^{+}$of $G^{\prime}\left(\mathfrak{g}=\mathfrak{t}+\bigoplus_{\xi \in \mathcal{R}^{+}} \mathfrak{g}_{\rho}\right)$. We denote by $\mathcal{B}:=\left(\xi_{1}, \ldots, \xi_{\mu}\right)$ a basis (or system of simple roots) of this system $\mathcal{R}^{+}$(such a basis exists). Then every root in $\mathcal{R}^{+}$is a linear combination of the roots 
of this basis with positive coefficients (they are integers) and therefore $\mathcal{B}$ is a good system of roots. Then the result follows from the proposition 5.18.

5.4. Some complements on linear algebraic groups. - We shall use later this part for the solution of the local inverse problem and in our study of the global inverse problem. Similar tools were introduced by the first author in order to solve inverse problems in the differential case. For the missing proofs $c f$. [36, 11.3,11.4].

We denote by $L(G)$ the subgroup of an algebraic group $G$ generated by all the maximal tori of $G$, it is a connected algebraic normal subgroup and the maximal torus of the algebraic group $V(G):=G / L(G)$ is reduced to the identity.

Lemma 5.22. - The Lie algebra $\mathfrak{L}$ of $L(G)$ is generated by $\mathfrak{t}$ (the Lie algebra of a maximal torus) and the root-spaces $\mathfrak{g}_{\xi}$.

The group $R_{u}(G) /\left(G^{0}, R_{u}(G)\right)$ is a commutative unipotent group therefore it can be identified with a finite dimensional complex vector space. The finite group $G / G^{0}$ acts naturally on $R_{u}(G) /\left(G^{0}, R_{u}(G)\right)$.

We set $S(G):=R_{u}(G) /\left(G^{0}, R_{u}(G)\right) \rtimes G / G^{0}$. Due to a result of the first author [36. Proposition 1.8, page 276], there is an isomorphim of algebraic groups:

$$
S(G) \rightarrow V(G) /\left(V(G)^{0}, V(G)^{0}\right) .
$$

Lemma 5.23. - The linear algebraic groups $S(G), V(G)$ and $V(G) /\left(V(G)^{0}, V(G)^{0}\right)$ have the same number $m$ of topological generators.

We have $\operatorname{dim} R_{u}(G) /\left(G^{0}, R_{u}(G)\right) \leq m$ and it is an equality if $G$ is connected. If $G$ is topologically generated by s elements, then $m \leq s$.

Lemma 5.24. - Let $G$ be an algebraic group endowed with a $\Theta$-structure defined by a $\Theta$-coweight $\chi$ on a Levi subgroup $D \subset G$. Let $T \subset D$ be the maximal torus of $D$. We set $U:=R_{u}(G)$ and denote $\mathfrak{u}$ its Lie algebra. Then:

(i) $\mathfrak{u}=\mathfrak{u}_{0} \oplus \bigoplus_{\xi \in \mathcal{R}} \mathfrak{g}_{\xi}$ and $\bigoplus_{\xi \in \mathcal{R}} \mathfrak{g}_{\xi}$ is a sub-Lie algebra of $\mathfrak{u}$;

(ii) $\mathfrak{L}=\mathfrak{t} \oplus \bigoplus_{\xi \in \mathcal{R}} \mathfrak{g}_{\xi}, \mathfrak{g}=\mathfrak{g}_{0} \oplus \bigoplus_{\xi \in \mathcal{R}} \mathfrak{g}_{\xi}, \mathfrak{g}_{0}=\mathfrak{u}_{0} \oplus \mathfrak{t}, \mathfrak{g}=\mathfrak{u}_{0} \oplus \mathfrak{L} ;$

Proof. - (i) For any weight $\xi$ on $D, \mathfrak{u}_{\xi} \subset \mathfrak{g}_{\xi}$ and if $\xi$ is a root, $\mathfrak{g}_{\xi}=\mathfrak{u}_{\xi}$.

Let $\alpha, \beta \in \mathcal{R}$, we have $\mathfrak{g}_{\alpha+\beta}=(0)$ or $\left[\mathfrak{g}_{\alpha}, \mathfrak{g}_{\beta}\right] \subset \mathfrak{g}_{\alpha+\beta}$. As $\alpha+\beta \neq 0$, in the second case $\alpha+\beta$ is a root. Hence $\bigoplus_{\xi \in \mathcal{R}} \mathfrak{g}_{\xi}$ is a sub-Lie algebra of $\mathfrak{u}$. 
(ii) For all $\xi \in \mathcal{R},\left[\mathfrak{t}, \mathfrak{g}_{\xi}\right]=\mathfrak{g}_{\xi}$, therefore, using (i) and the lemma 5.22, we get $\mathfrak{L}=\mathfrak{t} \oplus \bigoplus_{\xi \in \mathcal{R}} \mathfrak{g}_{\xi}$.

\section{The local inverse problem}

\subsection{The regular singular case. -}

6.1.1. Universal groups and representations. Necessary conditions. - The universal group for the local regular singular case (at 0) is the commutative proalgebraic group:

$$
G_{f}^{(0)}=G_{f, s}^{(0)} \times G_{f, u}^{(0)},
$$

with:

$$
G_{f, s}^{(0)}=\operatorname{Hom}_{g r}\left(\mathbf{E}_{q}, \mathbf{C}^{*}\right) \text { and } G_{f, s}^{(0)}=\mathbf{C} .
$$

To a germ (at the origin) of meromorphic $q$-difference system $\Delta: \sigma_{q} Y=$ $A Y$, up to meromorphic equivalence, corresponds a rational representation:

$$
\rho_{f}: G_{f}^{(0)} \rightarrow \mathrm{GL}_{n}(\mathbf{C})
$$

and conversely. The $q$-difference Galois group of $\Delta$ is $G=\operatorname{Im} \rho_{f}$. It is abelian.

The knowledge of the representation $\rho$ is equivalent to the knowledge of a pair of commuting representations:

$$
\rho_{f, s}: G_{f, s}^{(0)} \rightarrow \mathrm{GL}_{n}(\mathbf{C}) \quad \rho_{f, u}: G_{f, u}^{(0)} \rightarrow \mathrm{GL}_{n}(\mathbf{C}) .
$$

We have $G_{s}=\operatorname{Im} \rho_{f, s}$ and $G_{u}=\operatorname{Im} \rho_{f, u}$ and our commutation condition means that each element of $G_{s}$ commutes with each element of $G_{u}$.

The commutative unipotent group $G_{u}$ being the image of $\mathbf{C}$ by $\rho_{f, u}$ its dimension is at most one.

The group $\operatorname{Hom}_{g r}\left(\mathbf{E}_{q}, \mathbf{C}^{*}\right)$ is topologically generated by (exactly) two elements [30] and $\mathbf{C}$ is topologically generated by one element. Therefore the groups $G_{s}$ and $G$ are topologically generated by at most two elements, the group $G_{u}$ is topologically generated by one element. The finite group $G / G^{0}$ is algebraically generated by at most two elements.

Proposition 6.1. - Let $G$ be the Galois group of a local regular-singular q-difference system, then:

- $G$ is abelian and topologically generated by two elements;

- $G / G^{0}$ is algebraically generated by at most two elements;

$-\operatorname{dim}_{\mathbf{C}} G_{u} \leq 1$. 
6.1.1.1. A description of $\operatorname{Hom}_{g r}\left(\mathbf{E}_{q}, \mathbf{C}^{*}\right)$. - We recall the description of $\operatorname{Hom}_{g r}\left(\mathbf{E}_{q}, \mathbf{C}^{*}\right)$. We choose $\tau \in \mathbf{C}$ such that $e^{-2 i \pi \tau}=q(\operatorname{Im} \tau>0)$. The map $w \mapsto z:=e^{2 i \pi w}$ induces an isomorphism of $\mathbf{C} /(\mathbf{Z} \oplus \mathbf{Z} \tau)$ on $\mathbf{E}_{q}$. We consider $\mathbf{C}$ as a $\mathbf{Q}$-vector space, we can write it as a direct sum of $\mathbf{Q}$-vector spaces $\mathbf{C}=\mathbf{Q} \oplus \mathbf{Q} \tau \oplus \mathrm{E}$, then we have a product of $\mathbf{Z}$-modules $\mathbf{C} /(\mathbf{Z} \oplus \mathbf{Z} \tau) \approx$ $(\mathbf{Q} / \mathbf{Z}) \times(\mathbf{Q} \tau / \mathbf{Z} \tau) \times \mathrm{E}$ and the corresponding image is the product of $\mathbf{Z}$ modules:

$$
\mathbf{E}_{q}=\underline{\mu} \times \underline{\mu}_{q} \times \mathrm{E}
$$

where $\underline{\mu}:=e^{2 i \pi \mathbf{Q}}$ is the group of the roots of the unity, $\underline{\mu}_{q}=q^{\mathbf{Q}} / q^{\mathbf{Z}}$ is the image in $\mathbf{E}_{q}$ of the subgroup $q^{\mathbf{Q}} \subset \mathbf{C}^{*}\left(\underline{\mu}_{q} \approx \underline{\mu} \approx \mathbf{Q} / \mathbf{Z}\right)$ and $\mathrm{E}$ is a torsion free subgroup (the "universal lattice", defined up to isomorphism).

We shall consider each abelian group as the inductive limit of its finitely generated subgroups.

Recall that the groups written $G^{(0)}$ are (universal) local Galois groups at 0 , while $G^{0}$ means the neutral component of any proalgebraic group $G$. We have a short exact sequence of proalgebraic groups:

$$
\left(G_{f, s}^{(0)}\right)^{0} \rightarrow G_{f, s}^{(0)} \rightarrow G_{f, s}^{(0)} /\left(G_{f, s}^{(0)}\right)^{0},
$$

we get it applying the exact contravariant functor $\operatorname{Hom}_{g r}\left(., \mathbf{C}^{*}\right)$ to the short exact sequence of groups:

$$
\underline{\mu} \times \underline{\mu}_{q} \rightarrow \mathbf{E}_{q} \rightarrow \mathbf{E}_{q} /\left(\underline{\mu} \times \underline{\mu}_{q}\right) .
$$

We have $\mathbf{E}_{q} \rightarrow \mathbf{E}_{q} /\left(\underline{\mu} \times \underline{\mu}_{q}\right) \approx \mathrm{L}$, therefore:

$$
\operatorname{Hom}_{g r}\left(\mathbf{E}_{q} /\left(\underline{\mu} \times \underline{\mu}_{q}\right), \mathbf{C}^{*}\right) \approx \operatorname{Hom}_{g r}\left(\mathrm{E}, \mathbf{C}^{*}\right) .
$$

Hence $\left(G_{f, s}^{(0)}\right)^{0} \approx \operatorname{Hom}_{g r}\left(\mathbf{E}_{q} /\left(\underline{\mu} \times \underline{\mu}_{q}\right), \mathbf{C}^{*}\right)$ is a protorus, we will call it the fuchsian protorus and we will denote it $\mathbf{T}_{\mathbf{f}}$.

We recall that $\operatorname{Hom}_{g r}\left(\mathbf{Q} / \mathbf{Z}, \mathbf{C}^{*}\right)=\hat{\mathbf{Z}}$. Then $\operatorname{Hom}\left(\underline{\mu}, \mathbf{C}^{*}\right)=\hat{\mathbf{Z}}(1)(\hat{\mathbf{Z}}(1)$ is the multiplicative notation for $\hat{\mathbf{Z}})$.

We have $G_{f, s}^{(0)} / \mathbf{T}_{\mathbf{f}} \approx \hat{\mathbf{Z}}(1) \times \hat{\mathbf{Z}}(1)$.

Considering $G_{f, s}^{(0)}$ as a proalgebraic group, we get $\mathbf{E}_{q}=\operatorname{Hom}\left(G_{f, s}^{(0)}, \mathbf{C}^{*}\right)$ (here Hom is for morphisms of proalgebraic groups i.e. rational homomorphisms), as the inductive limit of its finitely generated subgroups. Then we can consider $\mathbf{E}_{q}$ as the group of weights of $G_{f, s}^{(0)}$. More precisely, if $\bar{c} \in \mathbf{E}_{q}$, then the map $\psi_{\bar{c}}: G_{f, s}^{(0)}=\operatorname{Hom}_{g r}\left(\mathbf{E}_{q}, \mathbf{C}^{*}\right) \rightarrow \mathbf{C}^{*}$ defined by $f \in \operatorname{Hom}_{g r}\left(\mathbf{E}_{q}, \mathbf{C}^{*}\right) \mapsto f(\bar{c})$ is a weight on $G_{f, s}^{(0)}$ and conversely if $\varphi$ is a weight on $G_{f, s}^{(0)}$, there exists a unique $\bar{c} \in \mathbf{E}_{q}$ such that $\varphi=\psi_{\bar{c}}$. 
Applying the functor $\operatorname{Hom}\left(., \mathbf{C}^{*}\right)$ to the (non-canonical) decomposition $G_{f, s}^{(0)}=\hat{\mathbf{Z}}(1) \times \hat{\mathbf{Z}}(1) \times \mathbf{T}_{\mathbf{f}}$, we get the (non-canonical) decomposition $\mathbf{E}_{q}=\underline{\mu} \times \underline{\mu}_{q} \times \mathrm{E}$.

6.1.2. The inverse problem for the regular-singular case, a tannakian solution. - We solve the inverse problem for the regular-singular case using the tannakian mechanism, proving that the conditions of the proposition 6.1 are sufficient. Afterwards we will give an elementary proof.

Proposition 6.2. - Let $G$ be an abelian complex linear algebraic group such that:

(i) $G$ is topologically generated by at most two elements;

(ii) $\operatorname{dim}_{\mathbf{C}} G_{u} \leq 1$.

Then $G$ is the local Galois group of a local regular singular meromorphic linear $q$-difference system.

The condition (i) can be replaced by the following (a priori weaker) condition:

(iii) $G / G^{0}$ is generated by at most two elements.

Proof. - We will give a tannakian proof, defining a surjective morphism $\rho$ : $G_{f, s}^{(0)} \rightarrow G$. Then, if $r: G \rightarrow \mathrm{GL}_{n}(\mathbf{C})$ is a faithful representation, the morphism $r \circ \rho: G_{f, s}^{(0)} \rightarrow \mathrm{GL}_{n}(\mathbf{C})$ defines a system of rank $n$ whose Galois group is $r(G)$.

Let $G$ be an abelian linear algebraic group satisfying the above conditions, then $G=G_{u} \times G_{s}$. The natural map $G_{s} / G_{s}^{0} \rightarrow G / G^{0}$ is an isomorphism, therefore there exists an isomorphism $G_{s} \approx \mathbf{Z} / p_{1} \mathbf{Z} \times \mathbf{Z} / p_{2} \mathbf{Z} \times\left(\mathbf{C}^{*}\right)^{\nu}\left(p_{1}, p_{2} \in\right.$ $\mathbf{N}^{*}$ ). We will suppose $p_{1}, p_{2} \geq 2$, leaving the other cases to the reader.

Using a sub-lattice of rank $n$ of $\mathrm{E}$, we get a surjective morphism $\mathbf{T}_{\mathbf{f}} \rightarrow\left(\mathbf{C}^{*}\right)^{\nu}$. There exists also a surjective morphism $\underline{\mu} \times \underline{\mu}_{q} \rightarrow \mathbf{Z} / p_{1} \mathbf{Z} \times \mathbf{Z} / p_{2} \mathbf{Z}$. Hence we get a surjective morphism

$$
\rho_{s}: G_{f, s}^{(0)} \approx \underline{\mu} \times \underline{\mu}_{q} \times \mathbf{T}_{\mathbf{f}} \rightarrow \mathbf{Z} / p_{1} \mathbf{Z} \times \mathbf{Z} / p_{2} \mathbf{Z} \times\left(\mathbf{C}^{*}\right)^{\nu} .
$$

The Lie algebra $\mathfrak{u}$ of $G_{u}$ is of dimension at most one. Therefore there exists $N \in \mathfrak{u}$ such that $G_{u}=\{\exp t N \mid t \in \mathbf{C}\}$. If $N=0$, the end of the proof is trivial. Otherwise, the map $\rho_{u}: G_{f, u}^{(0)} \approx \mathbf{C} \rightarrow G_{u}$ defined by $t \mapsto \exp t N$ is an isomorphism (of algebraic groups).

The representations $\rho_{s}$ and $\rho_{u}$ clearly commute and the morphism $\rho:=$ $\left(\rho_{s}, \rho_{u}\right): G_{f}^{(0)}=G_{f, s} \times G_{f, u} \rightarrow G$ is onto. That ends the proof. 
6.1.3. Explicit descriptions and elementary proof. - We shall recall how to compute the Galois group of a local regular-singular $q$-difference system and shall deduce a (elementary) proof of proposition 6.2 from this computation.

Up to a meromorphic gauge transformation, it is sufficient to consider the case of a constant coefficient system $\Delta: \sigma_{q} Y=A Y, A \in \mathrm{GL}_{n}(\mathbf{C})$.

We suppose that the matrix $A$ is in upper triangular Jordan form. The representation $\rho$ of the universal group $G_{f}^{(0)}=G_{f, s}^{(0)} \times G_{f, u}^{(0)}$ associated to the system $\Delta$ is:

$$
\rho=\left(\rho_{s}, \rho_{u}\right):(\gamma, \lambda) \mapsto \gamma\left(A_{s}\right) A_{u}^{\lambda} .
$$

We have $A_{s}=\operatorname{Diag}\left(a_{1}, \ldots, a_{n}\right)$, then $\gamma\left(A_{i, s}\right)=\operatorname{Diag}\left(\gamma\left(\bar{a}_{1}\right), \ldots, \gamma\left(\bar{a}_{n}\right)\right), \bar{a}_{i}$ being the image of $a_{i}$ in $\mathbf{E}_{q}$.

Let $H$ be the subgroup of $\mathbf{E}_{q}$ generated by the image of $\operatorname{Spec} A$. Using the decomposition $\mathbf{E}_{q}=\underline{\mu} \times \underline{\mu}_{\times} \mathrm{E}$, we get (up to the isomorphism $\underline{\mu}_{q} \approx \underline{\mu}$ ) $H=\mathbf{Z} / p_{1} \mathbf{Z} \times \mathbf{Z} / p_{2} \mathbf{Z} \times \Lambda$, where $\Lambda$ is a lattice of rank $\mu$.

The algebraic group $\operatorname{Hom}_{g r}\left(H, \mathbf{C}^{*}\right)$ is an algebraic quotient of $\operatorname{Hom}_{g r}\left(\mathbf{E}_{q}, \mathbf{C}^{*}\right)$ (using the canonical injection $H \rightarrow \mathbf{E}_{q}$ ) and the semi-simple component $G_{s}$ of the Galois group $G$ of $\Delta$ is the image of the quotient map, that is $\operatorname{Hom}_{g r}\left(H, \mathbf{C}^{*}\right)$, then:

$$
G_{s}=\mathbf{Z} / p_{1} \mathbf{Z} \times \mathbf{Z} / p_{2} \mathbf{Z} \times \operatorname{Hom}_{g r}\left(H, \mathbf{C}^{*}\right) \approx \mathbf{Z} / p_{1} \mathbf{Z} \times \mathbf{Z} / p_{2} \mathbf{Z} \times\left(\mathbf{C}^{*}\right)^{\mu} .
$$

More precisely we get the representation of $G_{s}$ in $\mathrm{GL}_{n}(\mathbf{C})$ corresponding to (8) using the interpretation of $H$ as the group of weights of $G_{s}$. This representation is given by the diagonal weights $\left(\bar{a}_{i}\right)_{i=1, \ldots, n}\left(\bar{a}_{i} \in H\right)$.

We can now solve explicitly the inverse problem.

Let $G$ be an abelian complex linear algebraic group satisfying the conditions of the proposition 6.2. we will compute a matrix $A \in \mathrm{GL}_{n}(\mathbf{C})$ such that the system $\Delta: \sigma_{q} Y=A Y$ admits $G$ as Galois group.

More precisely, we start from a faithful representation of the abelian group $G$ in $\mathrm{GL}_{n}$ in upper triangular form. Then $G_{s}$ is diagonal and (due to condition (ii)) there exists a unipotent matrix $N \in \mathcal{M}_{n}(\mathbf{C})$ such that $G_{u}=\left\{N^{\lambda} \mid \lambda \in\right.$ C\}.

The abelian linear algebraic group $G_{s}$ is isomorphic to the product of a finite group (the quotient $G / G^{0}$ ) by a torus of dimension $\mu$, and the finite component is generated by at most two elements. Then $G_{s} \approx \mathbf{Z} / p_{1} \mathbf{Z} \times \mathbf{Z} / p_{2} \mathbf{Z} \times\left(\mathbf{C}^{*}\right)^{\mu}$. The dual group (group of weights) of $\mathbf{Z} / p_{1} \mathbf{Z} \times \mathbf{Z} / p_{2} \mathbf{Z} \times\left(\mathbf{C}^{*}\right)^{\mu}$ is $\mathbf{Z} / p_{1} \mathbf{Z} \times \mathbf{Z} / p_{2} \mathbf{Z} \times \mathbf{Z}^{\mu}$ 
Using the decomposition $\mathbf{E}_{q}=\underline{\mu} \times \underline{\mu}_{q} \times \mathrm{E}$, we get an isomorphism between $\mathbf{Z} / p_{1} \mathbf{Z} \times \mathbf{Z} / p_{2} \mathbf{Z} \times \mathbf{Z}^{\mu}$ and a subgroup $H$ of $\mathbf{E}_{q}$. We can therefore interpret $H$ as the group of weights on the diagonal group $G_{s}$.

We denote $\varpi_{1}, \ldots, \varpi_{n}$ the diagonal weights of the diagonal group $G_{s}$, they are elements of $H$. Let $a_{1}, \ldots, a_{n} \in \mathbf{C}^{*}$ such that their natural images in $\mathbf{E}_{q}$ are $\varpi_{1}, \ldots, \varpi_{n}$. We moreover require these choices to be consistent in the following sense: each time $\varpi_{i}=\varpi_{j}$, we take $a_{i}=a_{j}$. Then $H$ is generated by $\bar{a}_{1}=\varpi_{1}, \ldots, \bar{a}_{n}=\varpi_{n}$.

We can now define $A \in \mathrm{GL}_{n}(\mathbf{C})$ :

$$
A_{s}:=\operatorname{Diag}\left(a_{1}, \ldots, a_{n}\right) \text { and } A_{u}:=N .
$$

Indded, because of our consistent choices above, $A_{s}$ and $A_{u}$ do commute. Then the Galois group of $\Delta: \sigma_{q} Y=A Y$ is $G$.

\subsection{The pure case with integral slopes. -}

6.2.1. Universal groups and representations. Necessary conditions. - The universal group for the pure case with integral slopes (at 0) is the commutative proalgebraic group:

$$
G_{p, 1}^{(0)}=G_{f, s}^{(0)} \times G_{f, u}^{(0)} \times T_{1}^{(0)}
$$

with:

$$
G_{f, s}^{(0)}=\operatorname{Hom}_{g r}\left(\mathbf{E}_{q}, \mathbf{C}^{*}\right), \quad G_{f, s}^{(0)}=\mathbf{C} \text { and } T_{1}^{(0)}=\mathbf{C}^{*} .
$$

To a germ (at the origin) of meromorphic $q$-difference system, pure with integral slopes, up to meromorphic equivalence, corresponds a morphism:

$$
\rho: G_{p, 1}^{(0)} \rightarrow \mathrm{GL}_{n}(\mathbf{C}) ;
$$

$G=\operatorname{Im} \rho$ is the Galois group of the system, it is commutative.

The knowledge of the representation $\rho$ is equivalent to the knowledge of a triple of pairwise commuting representations:

$$
\rho_{f, s}: G_{f, s}^{(0)} \rightarrow \mathrm{GL}_{n}(\mathbf{C}), \quad \rho_{f, u}: G_{f, u}^{(0)} \rightarrow \mathrm{GL}_{n}(\mathbf{C}), \quad \rho_{\theta}: T_{1}^{(0)} \rightarrow \mathrm{GL}_{n}(\mathbf{C})
$$

We have (up to the obvious reordering of the factors) $G_{s}=\operatorname{Im}\left(\rho_{f, s}, \rho_{\theta}\right), G_{u}=$ $\operatorname{Im} \rho_{f, u}$.

As in the regular singular case, we get the following result.

Proposition 6.3. - Let $G$ be the Galois group of a local pure q-difference system, then:

- $G$ is abelian and topologically generated by two elements;

- $G / G^{0}$ is algebraically generated by at most two elements;

$-\operatorname{dim}_{\mathbf{C}} G_{u} \leq 1$. 
We recall that we have a (non-canonical) decomposition $G_{f, s}^{(0)}=\hat{\mathbf{Z}}(1) \times$ $\hat{\mathbf{Z}}(1) \times \mathbf{T}_{\mathbf{f}}$, where the fuchsian universal protorus is a subgroup of $G_{f, s}^{(0)}$.

We will denote $T_{f}$ the image of $\mathbf{T}_{\mathbf{f}}$ by $\rho_{f, s}$ and we will call it the fuchsian torus of $G$. We will denote $T_{\theta}$ the image of $\mathbf{T}_{\mathbf{1}}{ }^{(0)}$ by $\rho_{f, s}$ and we will call it the $\theta$-torus of $G$. The $\theta$-torus and the fuchsian torus of $G$ generate the maximal torus of $G$.

\subsubsection{Sufficient conditions. -}

Proposition 6.4. - Let $G$ be an abelian complex linear algebraic group and a non trivial coweight $\chi: \mathbf{C}^{*} \rightarrow G_{s}$. We suppose that:

(i) $G$ is topologically generated by at most two elements;

(ii) $\operatorname{dim}_{\mathbf{C}} G_{u} \leq 1$.

Then $G$ is the local Galois group of a local pure meromorphic linear qdifference system with integral slopes such that $\chi=\rho_{\theta}$ (where $\rho=\left(\rho_{f}, \rho_{\theta}\right)$ is the representation defining the system). The condition (i) can be replaced by the following (a priori weaker) condition:

(iii) $G / G^{0}$ is generated by at most two elements.

Proof. - We will prove the existence of a system such that its fuchsian torus $T_{f}$ is a maximal torus, or equivalently such that $T_{\theta} \subset T_{f}$. The proof is tannakian and it is only a slight modification of the proof of the proposition 6.2 ,

Let $T$ be the maximal torus of $G$, it contains the image of $\chi$.

We build as above a surjective representation:

$$
\rho_{f}=\left(\rho_{f, s}, \rho_{f, u}\right): G_{f}^{(0)}=G_{f, s}^{(0)} \times G_{f, u}^{(0)} \rightarrow G .
$$

Then using $\mathbf{T}_{1}^{(0)}=\mathbf{C}^{*}$, we define a representation

$$
\rho=\left(\rho_{f}, \rho_{\theta}\right): G_{p, 1}^{(0)}=G_{f}^{(0)} \times \mathbf{T}_{1}^{(0)} \rightarrow G,
$$

by $\rho_{\theta}=\chi$. (The component representations automatically commute.) It is a surjective morphism and it answers the question.

6.2.3. Explicit descriptions. - We recall how to compute the Galois group of a local pure $q$-difference system with integral slopes and deduce a new (elementary) proof of proposition 6.4 from this computation. 
Up to a meromorphic gauge transformation, it is sufficient to consider the case of a system $\Delta: \sigma_{q} Y=A Y$, such that the matrix $A$ is in upper triangular normal form:

$$
A:=\left(\begin{array}{ccccc}
z^{\mu_{1}} A_{1} & \ldots & \ldots & \ldots & \ldots \\
\ldots & \ldots & \ldots & 0 & \ldots \\
0 & \ldots & \ldots & \ldots & \ldots \\
\ldots & 0 & \ldots & \ldots & \ldots \\
0 & \ldots & 0 & \ldots & z^{\mu_{k}} A_{k}
\end{array}\right)
$$

where, for $1 \leq i \leq k, A_{i} \in \mathrm{GL}_{r_{i}}(\mathbf{C})$ is in Jordan form, and $\mu_{1}, \ldots, \mu_{k} \in \mathbf{Z}$. (Usually we take $\mu_{1}<\cdots<\mu_{k}$, although this has no consequence in the formal case.)

The representation $\rho$ of the universal group $G_{p, 1}^{(0)}=G_{f, s}^{(0)} \times G_{f, u}^{(0)} \times T_{1}^{(0)}$ associated to the system $\Delta$ is:

$$
\rho=\left(\rho_{s}, \rho_{u}, \rho_{\theta}\right):(\gamma, \lambda, t) \mapsto\left(\begin{array}{ccccc}
t^{\mu_{1}} \gamma\left(A_{1, s}\right) A_{1, u}^{\lambda} & \ldots & \ldots & \ldots & \ldots \\
\ldots & \ldots & \ldots & 0 & \ldots \\
0 & \ldots & \ldots & \ldots & \ldots \\
\ldots & 0 & \ldots & \ldots & \ldots \\
0 & \ldots & 0 & \ldots & t^{\mu_{k}} \gamma\left(A_{k, s}\right) A_{k, u}^{\lambda}
\end{array}\right) \text {. }
$$

We can now give a new proof of proposition 6.4. We start from the abelian group $G$ and the one-parameter subgroup $\chi$. We can assume that it is diagonalized:

$$
\forall t \in \mathbf{C}^{*}, \chi(t)=\left(\begin{array}{ccccc}
t^{\mu_{1}} I_{r_{1}} & \ldots & \ldots & \ldots & \ldots \\
\ldots & \ldots & \ldots & 0 & \ldots \\
0 & \ldots & \ldots & \ldots & \ldots \\
\ldots & 0 & \ldots & \ldots & \ldots \\
0 & \ldots & 0 & \ldots & t^{\mu_{k}} I_{r_{k}}
\end{array}\right)
$$

and we apply the explicit proof of proposition 6.2 to each of the regular-singular blocks of ranks $r_{i}$, yielding matrices $A_{i}$ with constant coefficients. Then we set:

$$
A:=\left(\begin{array}{ccccc}
z^{\mu_{1}} A_{1} & \ldots & \ldots & \ldots & \ldots \\
\ldots & \ldots & \ldots & 0 & \ldots \\
0 & \ldots & \ldots & \ldots & \ldots \\
\ldots & 0 & \ldots & \ldots & \ldots \\
0 & \ldots & 0 & \ldots & z^{\mu_{k}} A_{k}
\end{array}\right)
$$


The image of $\chi$ is contained in $G$, therefore the Galois group of the system $\sigma_{q} Y=A Y$ is $G$ and we have $\chi=\rho_{\theta}$.

\subsection{The local inverse problem: the general case with integral slopes.}

\subsubsection{Necessary conditions. -}

Theorem 6.5. - Let $G$ be a complex linear algebraic subgroup. If $G$ is the local Galois group of a meromorphic linear q-difference equation, then:

(i) $G$ is triangularizable;

(ii) $G / L(G)$ is abelian and topologically generated by at most two elements;

(iii) the finite group $G / G^{0}$ is abelian and generated by at most two elements;

(iv) the group $G / G^{0}$ acts trivially on $R_{u}(G) /\left(G^{0}, R_{u}(G)\right)$ and the dimension of the vector space $R_{u}(G) /\left(G^{0}, R_{u}(G)\right)$ is at most one.

Proof. -

(i) Trivial.

(ii) We will use a Tannakian argument which is a variant of an idea due to O. Gabber in the differential case $\mathbf{1 3}$.

Let $G^{(0)}$ be the Tannakian group of the Tannakian category $\mathcal{E}^{(0)}$. To a $q$-difference system of rank $n$, meromorphic at the origin, corresponds a (rational) representation $\rho: G^{(0)} \rightarrow \mathrm{GL}_{n}(\mathbf{C})$ and conversely. If $G=$ $\operatorname{Gal}_{\mathbf{C}(\{z\})}(\Delta)$ is the Galois group of $\Delta$, then $G=\operatorname{Im} \rho$.

Let $\pi: G \rightarrow G / L(G)$ be the canonical map, let $\iota: G / L(G) \rightarrow \mathrm{GL}_{n^{\prime}}(\mathbf{C})$ be a faithful linear representation of $G / L(G)$, then we get a continuous linear representation $\rho^{\prime}: \iota \circ \pi \circ \rho: G^{(0)} \rightarrow \mathrm{GL}_{n^{\prime}}(\mathbf{C})$.

To the representation $\rho^{\prime}$ corresponds a $q$-difference system $\Delta^{\prime}$ of rank $n^{\prime}$ and and $G^{\prime}:=\iota(G / V(G))=\operatorname{Gal}_{\mathbf{C}(\{z\})}\left(\Delta^{\prime}\right)=\operatorname{Im} \rho^{\prime}$.

The maximal torus of $G^{\prime}$ is reduced to the identity, therefore the $\theta$ torus of $\Delta^{\prime}$ is trivial and $\Delta^{\prime}$ is regular singular.

Hence the Galois group $G^{\prime}$ of $\Delta^{\prime}$ is abelian and topologically generated by at most two elements [30]. Moreover $G^{\prime}=G_{s}^{\prime} G_{u}^{\prime}$, where the unipotent group $G_{u}^{\prime}$ is topologically generated by at most one element [30].

(iii) We have $G / L(G)=V(G) \approx G^{\prime}$. The group $G / G^{0}$ is a quotient of $V(G)$ therefore it is abelian and topologically generated by at most two elements, as it is finite it is algebraically generated by at most two elements.

- (iv) We set as in section $5.4 S(G):=R_{u}(G) /\left(G^{0}, R_{u}(G)\right) \rtimes G / G^{0}$, we recall that there is an isomorphim of algebraic groups $S(G) \rightarrow$ 
$V(G) /\left(V(G)^{0}, V(G)^{0}\right)$. The group $V(G)$ being commutative, we get an isomorphism $S(G) \rightarrow V(G), S(G)$ is commutative and the action of $G / G^{0}$ on $R_{u}(G) /\left(G^{0}, R_{u}(G)\right)$ is trivial.

We have an isomorphism $S(G)_{u}=R_{u}(G) /\left(G^{0}, R_{u}(G)\right) \rightarrow V(G)_{u}$. As $V(G)_{u}, S(G)_{u}$ is topologically generated by at most one generator. Then $\operatorname{dim}_{\mathbf{C}} R_{u}(G) /\left(G^{0}, R_{u}(G)\right) \leq 1$.

We think that the four necessary conditions of the above theorem are not sufficient. Anyway if we want to realize $G$ as the Galois group of a meromorphic linear $q$-difference system whose Newton polygon has integral slopes, then there is a new necessary condition ((vi) of the following theorem). This condition is not trivial: there exists a solvable linear algebraic group satisfying the conditions (ii), (iii), (iv) of theorem 6.5 which does not satisfies the condition (vi) of theorem 6.6 below ( $c f$. 3 of remark 5.16, page 49).

Theorem 6.6. - Let $G$ be a complex linear algebraic subgroup. If $G$ is the local Galois group of a meromorphic linear q-difference system whose Newton polygon has integral slopes, then:

(i) $G$ is triangularizable;

(ii) $G / L(G)$ is is abelian and topologically generated by at most two elements;

(iii) $G / G^{0}$ is abelian and generated by at most two elements;

(iv) the dimension of the unipotent component ot the abelian group $G / L(G)$ is at most one;

(v) the dimension of $R_{u}(G) /\left(G^{0}, R_{u}(G)\right)$ is at most one;

(vi) there exists a $\Theta$-structure on $G$.

Proof. - Assertions (i) to (v) follow from the proposition 6.1 and theorem 6.5 .

It remains to prove (vi).

Every system with integral slopes admits, up to meromorphic equivalence, a Birkhoff-Guenther normal form, therefore it is sufficient to prove the result for a system $\sigma_{q} Y=A Y$ in Birkhoff-Guenther normal form: 


$$
A=A_{U}:=\left(\begin{array}{ccccc}
B_{1} & \ldots & \ldots & \ldots & \ldots \\
\ldots & \ldots & \ldots & U_{i, j} & \ldots \\
0 & \ldots & \ldots & \ldots & \ldots \\
\ldots & 0 & \ldots & \ldots & \ldots \\
0 & \ldots & 0 & \ldots & B_{k}
\end{array}\right)
$$

where, for $1 \leq i<j \leq k, U_{i, j} \in \operatorname{Mat}_{r_{i}, r_{j}}(\mathbf{C}(\{z\}))$. Here, $U$ stands short for $\left(U_{i, j}\right)_{1 \leq i<j \leq k} \in \prod_{1 \leq i<j \leq k} \operatorname{Mat}_{r_{i}, r_{j}}(\mathbf{C}(\{z\}))$. (This requirement is actually weaker than the true Birkhoff-Guenther normal form, where the $U_{i, j}$ would have polynomial coefficients, $c f$. section 2.2 .)

We suppose that:

$$
B_{i}=z^{\mu_{i}} A_{i}, A_{i} \in \mathrm{GL}_{r_{i}}(\mathbf{C}), \mu_{1}<\cdots<\mu_{i}<\cdots<\mu_{k},
$$

and we set ((15) ):

$$
A_{0}:=\left(\begin{array}{ccccc}
B_{1} & \ldots & \ldots & \ldots & \ldots \\
\ldots & \ldots & \ldots & 0 & \ldots \\
0 & \ldots & \ldots & \ldots & \ldots \\
\ldots & 0 & \ldots & \ldots & \ldots \\
0 & \ldots & 0 & \ldots & B_{k}
\end{array}\right)
$$

We firstly consider the differential Galois group $G_{0}$ of the pure system $\sigma_{q} Y=$ $A_{0} Y$. This group is abelian, in upper triangular form, and its semi-simple component $D:=\left(G_{0}\right)_{s}$ is diagonal.

We define a coweight of $D$ by $\chi: t \mapsto\left(t^{\mu_{1}} I_{r_{1}}, \ldots, t^{\mu_{k}} I_{r_{k}}\right)$ and we denote its image by $T_{\theta}$ (the theta-torus). Then the maximal torus $T$ of $D$ is generated by the "fuchsian torus" $T_{f}$ and $T_{\theta}$.

We consider now the differential Galois group $G$ of the system $\sigma_{q} Y=A Y$. It is on upper triangular form, it contains $G_{0}$ as a subgroup, moreover $D$ is a Levi subgroup of $G$.

We denote $\varpi_{1}, \ldots, \varpi_{n}$ the diagonal weights of $D$. The root for the adjoint action of $D$ on $\mathfrak{g}$ are elements of the set $\left\{\varpi_{i} \varpi_{j}^{-1} \mid i<j\right\}$. If $\xi$ is a root, the corresponding root space is not trivial: there exists $x \in \mathfrak{g}_{\xi}$ such that $x \neq 0$. Therefore there exists $i, j$, with $i<j$, such that $x_{i j} \neq 0$, then $\langle\xi, \chi>=$ $\mu_{i}-\mu_{j}<0\left(\varpi_{i} \circ \chi(t)=t^{\mu_{i}}\right)$. Hence $\chi$ is a $\Theta$-structure on $G$.

6.3.2. Sufficient conditions. - We will prove in this part that the conditions of the theorem 6.6 are sufficient.

From lemma 5.24 we deduce the following preliminary result. 
Lemma 6.7. - Let $G$ be a complex linear algebraic group admitting a $\Theta$ structure. Then the following conditions are equivalent:

(i) the dimension of $R_{u}(G) /\left(G^{0}, R_{u}(G)\right)$ is at most one;

(ii) if $G=U \rtimes D$ is a Levi decomposition, then the dimension of $\mathfrak{u}_{0}$ (which was defined in lemma 5.24) is at most one;

(iii) if $D$ is a Levi subgroup of $G$, then $\operatorname{dim} C_{G}(D) \leq \operatorname{dim} D+1$ (we write $C_{G}(D)$ the centralizer of $\left.D\right)$.

If these conditions are satisfied, then:

$$
\operatorname{dim} R_{u}(G) /\left(G^{0}, R_{u}(G)\right)=\operatorname{dim} \mathfrak{u}_{0}=\operatorname{dim} C_{G}(D)-\operatorname{dim} D .
$$

Theorem 6.8. - Let $G$ be a complex linear algebraic group such that:

(i) $G / L(G)$ is abelian and topologically generated by at most two elements;

(ii) the dimension of $R_{u}(G) /\left(G^{0}, R_{u}(G)\right)$ is at most one;

(iii) $G$ admits a $\Theta$-structure,

then $G$ is the local Galois group of a meromorphic linear q-difference system whose Newton polygon has integral slopes.

More precisely, if $r: G \rightarrow G L_{n}(\mathbf{C})$ is a faithful representation, it is possible to find a meromorphic linear q-difference system whose Newton polygon has integral slopes and whose Galois group is $r(G)$.

It is possible to replace the condition (ii) by the following:

(ii') the dimension of the unipotent component of the abelian group $G / L(G)$ is at most one.

Proof. - The proof is tannakian, starting from an algebraic group $G$, we will obtain the system as a rational representation of the total Galois group with integral slopes:

$$
\rho=\left(\rho_{w}, \rho_{p, 1}\right): G_{1}^{(0)}=\mathfrak{S t} \rtimes G_{p, 1}^{(0)} \rightarrow G
$$

whose image is $G$.

We will built this representation using our main result on the description of the representations of the Tannakian group $G_{1}^{(0)}$ via the representations of the wild fundamental group $L \rtimes G_{p, 1, s}^{(0)}$. We recall ( $c f$. sections 2 and 3) that the knowledge of $\rho_{w}$ is equivalent to the knowledge of its infinitesimal counterpart $L \rho_{w}$ and that the knowledge of $L \rho_{w}$ is equivalent to the knowledge of a representation of $L: \lambda: L \rightarrow \mathfrak{g}$, compatible with the corresponding adjoint actions of $G_{p, 1, s}^{(0)}$ and $\rho_{p, 1}\left(G_{p, 1, s}^{(0)}\right)$ (cf. section 2.4). Moreover we have $\operatorname{Im} L \rho_{w}=\operatorname{Im} \lambda$. 
Let $G$ be a triangularizable complex linear algebraic goup. Let $\chi$ be a $\Theta$ coweight on a Levi subgroup $D$ of $G$. Using lemma 5.15, we can suppose that $\chi$ is dominant.

We will build the representation $\rho$ in three steps:

- we will define a rational representation $\rho_{f}: G_{f}^{(0)} \rightarrow G$, whose image is the centralizer $C_{G}(D)$;

- using the coweight $\chi: \mathbf{C}^{*} \rightarrow D$ and the canonical injection $D \rightarrow G$, we get a morphism $\rho_{\theta}: \mathbf{C}^{*} \rightarrow G$ and we define a rational representation $\rho_{p, 1}=\left(\rho_{\theta}, \rho_{f}\right): G_{p, 1}^{(0)} \rightarrow G$;

- we will define a representation $\lambda: L \rightarrow \mathfrak{g}$ such that, if $L \rho_{w}: \mathfrak{s t} \rightarrow \mathfrak{g}$ is the associated representation, then $\rho:=\left(\rho_{w}, \rho_{p, 1}\right): G_{1}^{(0)} \rightarrow G$ is onto.

6.3.2.1. Definition of $\rho_{f}$ and $\rho_{p, 1}$. - We consider the centralizer $C_{G}(D)$, its Lie algebra is $\mathfrak{u}^{0} \oplus \mathfrak{t}$ and, according to the hypothesis and to lemma6.7, $\operatorname{dim} \mathfrak{u}^{0} \leq$ 1. We choose a generator $N$ of the vector space $\mathfrak{u}^{0}$, then $C_{G}(D)=U_{0} \times D$, where $U_{0}=\exp \mathfrak{u}^{0}=\{\exp t N \mid t \in \mathbf{C}\}$, in particular $C_{G}(D)$ is abelian.

We consider the abelian algebraic group $C_{G}(D)$ and the coweight $\chi$ on $D \subset$ $C_{G}(D)$. They satisfy the conditions of proposition $6.3\left(C_{G}(D) /\left(C_{G}(D)\right)^{0} \approx\right.$ $\left.G / G^{0}\right)$, therefore there exists a representation

$$
\rho_{p, 1}^{\prime}: G_{p, 1}^{(0)} \rightarrow C_{G}(D)
$$

such that $\operatorname{Im} \rho_{p, 1}^{\prime}=C_{G}(D)$ and such that the corestriction of $\rho_{\theta}^{\prime}: \mathbf{T}_{1}^{(0)} \rightarrow$ $C_{G}(D)$ to $D$ is equal to the coweight $\chi$.

By composition of $\rho_{p, 1}^{\prime}$ by the canonical injection $C_{G}(D) \rightarrow G$, we get a representation:

$$
\rho_{p, 1}=\rho_{p, 1}^{\prime} \circ r: G_{p, 1}^{(0)} \rightarrow G .
$$

Its image is topologically generated by $D$ and $\exp N$.

6.3.2.2. Definition of $\rho_{w}$ and $\rho$. - We want to extend the representation $\rho_{p, 1}$ into a surjective representation

$$
\rho=\left(\rho_{w}, \rho_{p, 1}\right): G_{1}^{(0)}=\mathfrak{S} \rtimes G_{p, 1}^{(0)} \rightarrow G .
$$

As we recalled above, the knowledge of $\rho_{w}: \mathfrak{S t} \rightarrow G$ is equivalent to the knowledge of a representation:

$$
\lambda: L \rightarrow \mathfrak{g},
$$

the images of $\lambda$ and $L \rho_{w}$ being equal. 
We will define $\lambda$ such that its image contains all the root spaces $\mathfrak{g}_{\xi}, \xi \in \mathcal{R}$.

We have a surjective map

$$
\rho_{f, s}: G_{f, s}^{(0)}=\operatorname{Hom}_{g r}\left(\mathbf{E}_{q}, \mathbf{C}^{*}\right) \rightarrow D .
$$

Let $\xi$ be a root on $D$, then $\xi \circ \rho_{f, s}$ defines a weight on $\operatorname{Hom}_{g r}\left(\mathbf{E}_{q}, \mathbf{C}^{*}\right)$, that is an element $\bar{c} \in \mathbf{E}_{q}$. We set $\langle\xi, \chi\rangle=:-\delta, \delta \in \mathbf{N}^{*}$. Therefore to each root $\xi$ we associate a label $(\delta, \bar{c}) \in \mathbf{N}^{*} \times \mathbf{E}_{q}$. We denote by $\Sigma \subset \mathbf{N}^{*} \times \mathbf{E}_{q}$ the finite subset of labels obtained from the roots by this procedure.

If $(\delta, \bar{c}) \notin \Sigma$, for all $i=1, \ldots, \delta$, we set $\lambda\left(\dot{\Delta}_{i}^{(\delta, \bar{c})}\right):=0$. It remains to define $\lambda\left(\dot{\Delta}_{i}^{(\delta, \bar{c})}\right)$ for $(\delta, \bar{c}) \in \Sigma$ and for all $i=1, \ldots, \delta$.

We set $d_{\xi}:=\operatorname{dim} \mathfrak{g}_{\xi}$. The $\Theta$-coweight $\chi$ is dominant, therefore $d_{\xi} \leq \delta$. We choose a basis $\left(e_{\xi, 1}, \ldots, e_{\xi, d_{\xi}}\right)$ of the vector space $\mathfrak{g}_{\xi}$ and we set $\lambda\left(\dot{\Delta}^{(\delta, \bar{c})}\right):=e_{\xi, i}$ if $i=1, \ldots, d_{\xi}$ and $\left.\dot{\Delta}_{i}^{(\delta, \bar{c})}\right):=0$ if $i=d_{\xi}+1, \ldots, \delta$.

Then, for every root $\xi \in \mathcal{R}$, the image of $\lambda$ contains the root space $\mathfrak{g}_{\xi}$.

6.3.2.3. End of the proof. - By construction, the image of $\lambda$, and therefore the image of $L \rho_{w}$ contains the sum of the root spaces $\bigoplus_{\xi \in \mathcal{R}} \mathfrak{g}_{\xi}=\bigoplus_{\xi \in \mathcal{R}} \mathfrak{u}_{\xi}$ and the image of $L \rho_{f, u}$ is $u_{0}$. Therefore the image of $L \rho$ contains $\mathfrak{u}$ and the image of $\rho$ contains $U=R_{u}(G)$. The image of $\rho$ contains also $C_{G}(D)$ and a fortiori $D$. Finally the image of $\rho$ is $G$.

Using proposition 5.21 we get the following result.

Corollary 6.9. - Let $G$ be a Borel subgroup of a connected reductive algebraic group, then it is the local Galois group of a meromorphic linear qdifference system whose Newton polygon has integral slopes.

\section{About the global inverse problem}

We have a "glueing" lemma.

Lemma 7.1. - (i) Let $A^{(0)}\left(\right.$ resp. $\left.A^{(\infty)}\right)$ be an object of $\mathcal{E}_{1}^{(0)}\left(\right.$ resp. $\left.\mathcal{E}_{1}^{(\infty)}\right)$. We suppose that $A^{(0)}$ and $A^{(\infty)}$ are in Birkhoff-Guenther normal form and that $A_{f}^{(0)}=A_{f}^{(\infty)} \in G L_{n}(\mathbf{C})$. Let $G_{1}^{(0)}$ (resp. $G_{1}^{(\infty)}$ ) be the Galois group of $A^{(0)}$ (resp. $\left.A^{(\infty)}\right)$ and $G$ the Galois group of the global system defined by $\left(A^{(0)}, I_{n}, A^{(\infty)}\right)$. Then $G$ is the Zariski closure in $G L_{n}(\mathbf{C})$ of the subgroup generated by $G_{1}^{(0)}$ and $G_{1}^{(\infty)}$.

(ii) Let $G^{+}$and $G^{-}$be two connected algebraic subgroups of $G L_{n}(\mathbf{C})$ satisfying the conditions of theorem 6.8 (or equivalently such that they are local 
Galois group of meromorphic linear q-difference systems whose Newton polygon have integral slopes). We suppose that $G^{+}$and $G^{-}$admit a same maximal torus. We denote $G$ the Zariski closure in $G L_{n}(\mathbf{C})$ of the subgroup generated by $G^{+}$and $G^{-}$. Then $G$ is the global Galois group of a meromorphic linear q-difference system whose Newton polygon has integral slopes at 0 and $\infty$.

Proof. - (i) follows easily from theorem 4.5.

(ii) Going back to the proof of theorem 6.8, we can find $A^{(0)}$ (reps. $A^{(\infty)}$ ) such that $G^{+}$(reps. $G^{-}$) is the Galois group of $A^{(0)}$ (reps. $A^{(\infty)}$ ) and such that $A_{f}^{(0)}=A_{f}^{(\infty)}$ (we choose $A_{f}^{(0)}$ such that the subgroup generated by its semi-simple part is Zariski dense in $T$ ). Then the result follows from (i).

Proposition 7.2. - Let $G$ be a connected reductive linear algebraic group, then $G$ is the global Galois group of a meromorphic linear q-difference system whose Newton polygons at 0 and $\infty$ have integral slopes.

Moreover it is possible to get a q-difference system admitting $G$ as a Galois group with a trivial (generalized) Birkhoff connection matrix and such that the local groups at 0 and $\infty$ are Borel subgroups.

Proof. - If the maximal torus of $G$ is trivial, then the conditions of the proposition 6.1 are satisfied, therefore $G$ is the Galois group of a local regular singular equation. It is easy to conclude using [30].

We can suppose that $G \subset \mathrm{GL}_{n}(\mathbf{C})$ and that the maximal torus $T$ of $G$ is not trivial and in diagonal form.

We denote $G^{+}$and $G^{-}$two opposite Borel subgroups of $G$ and we choose as explained above a coweight $\chi$ of $T$ such that $\chi$ is a $\Theta$-coweight for $G^{+}$and $\chi^{-1}$ is a $\Theta$-coweight for $G^{-}$. Using 6.9 we prove that $G^{+}$(resp. $G^{-}$) is the local Galois group of a meromorphic linear $q$-difference system whose Newton polygon has integral slopes. We end the proof using lemma 7.1

Theorem 7.3. - Let $G$ be a connected linear algebraic group,. We suppose that the dimension of the vector space $R_{u}(G) /\left(G, R_{u}(G)\right)$ is at most 2 . Then $G$ is the global Galois group of a rational linear q-difference system whose Newton polygons at 0 and $\infty$ have integral slopes.

In particular we can apply this result to a connected group. It generalizes proposition 7.2 . 
Proof. - If the maximal torus of $G$ is trivial, then the conditions of proposition 6.1 are satisfied, therefore $G$ is the Galois group of a local regular singular equation. It is easy to conclude using [30].

We can suppose that $G \subset \mathrm{GL}_{n}(\mathbf{C})$ and that the maximal torus $T$ of $G$ is not trivial and in diagonal form.

Lemma 7.4. - There exists a coweight $\chi$ on $T$ which is non null on each root $\xi$ for the adjoint action of $T$ on the Lie algebra $\mathfrak{g}$ of $G:\langle\xi, \chi\rangle \neq 0$.

Proof. - The proof is a variant of an argument used above.

Let $\Phi:\left(\mathbf{C}^{*}\right)^{\mu} \rightarrow T$ be an isomorphism of tori.

Let $\Sigma=\left\{\xi_{1}, \ldots, \xi_{\nu}\right\}$ be the set of of roots of $G$. For $i=1, \ldots, \nu$, we set $f_{i}:=L \xi_{i} \circ L \Phi$. We interpret $f_{1}, \ldots, f_{\mu^{\prime}}$ as linear forms on $\mathbf{R}^{\mu}$. There exists $p=\left(p_{1}, \ldots, p_{\mu}\right) \in \mathbf{Z}^{\mu}$ such that $f_{i}(p) \neq 0$ for all $i=1, \ldots, \nu$ (cf. lemma 5.19). We define a coweight $\chi: \mathbf{C}^{*} \rightarrow T$ by $\Phi^{-1} \circ \chi: t \mapsto\left(t_{1}:=t^{p_{1}}, \ldots, t_{\mu}:=t^{p_{\mu}}\right)$, then, for $i=1, \ldots, \nu$, we set $v_{i}:=f_{i} \circ L\left(\Phi^{-1} \circ \chi\right):=L \xi_{i} \circ L \chi$. We have $v_{i}(1)=f_{i}(p) \neq 0$, then $\left\langle L \xi_{i}, L \chi>=<\xi_{i}, \chi>\neq 0\right.$.

We return to the proof of the theorem. We will suppose that we are in the "worst case" that is $\operatorname{dim} R_{u}(G) /\left(G, R_{u}(G)\right)=2$, the reader will easily adapt the proof to the other cases.

We denote by $\mathcal{R} \subset \mathfrak{g}^{*}$ the set of roots.

The commutative group $V(G) \approx R_{u}(G) /\left(G, R_{u}(G)\right)$ is topologically generated by two elements ( $c f$. lemma 5.23).

The Lie algebra of $V(G)$ is the image of $\mathfrak{g}_{0}^{n}$ induced by the quotient map (cf. lemma 5.22). Hence there exist $N^{+}, N^{-} \in \mathfrak{g}_{0}^{n}$ whose images generate the Lie algebra of $V(G)$. Then the Lie algebra $\mathfrak{g}$ is generated by $\mathbf{C} N^{+}, \mathbf{C} N^{-}$and the Lie algebra of $L(G)$, therefore by $\mathbf{C} N^{+}, \mathbf{C} N^{-}, \mathfrak{t}$ and the root spaces $\mathfrak{g}_{\xi}$, $\xi \in \mathcal{R}$ ( $c f$. lemma 5.22 ).

We set $\mathcal{R}^{+}:=\{\xi \in \mathcal{R} \mid<\xi, \chi><0\}$ and $\mathcal{R}^{-}:=\{\xi \in \mathcal{R} \mid<\xi, \chi>>0\}$. We have a partition $\mathcal{R}=\mathcal{R}^{+} \cup \mathcal{R}^{-}$.

We denote by $G^{+}$(reps. $G^{-}$) the algebraic subgroup of $G$ topologically generated by, $T, \exp \left(\mathbf{C} N^{+}\right)$and the $\exp \mathfrak{g}_{\xi}, \xi \in \mathcal{R}^{+}\left(\right.$resp. $T, \exp \left(\mathbf{C} N^{-}\right)$ and the exp $\mathfrak{g}_{\xi}, \xi \in \mathcal{R}^{-}$). The group $G$ is clearly topologically generated by $G^{+}$and $G^{-}$.

Then $\chi$ defines a $\Theta$-structure on $G^{+}$and $\chi^{-1}$ defines a $\Theta$-structure on $G^{-}$. Using 6.8 we prove that $G^{+}$(resp. $G^{-}$) is the local Galois group of a meromorphic linear $q$-difference system whose Newton polygon has integral slopes. We end the proof using lemma 7.1 . 
Remark 7.5. - In fact as we noticed above, we proved more than what is stated in the proposition. In some sense the only singularities of the constructed equation are 0 and $\infty(c f .[29])$. This is a first step towards a $q$-analog version of the Abhyankar conjecture. The reader will compare with the solution of the differential Abhyankar conjecture due to the first author.

Theorem 7.6. - If a complex linear algebraic group $G$ is the q-difference Galois group of a rational system, then $V(G):=G / L(G)$ is the q-difference Galois group of a rational regular-singular system.

The proof is "Tannakian" and similar to the first part of the proof of the theorem 6.5

Conversely we can conjecture that, using a variant of the proof of the proposition 7.3, the condition of the theorem is not only necessary but that it is also sufficient (the reader will compare with the proof of the corresponding result in the differential case by the first author).

\section{Appendix A}

\section{Pronilpotent completions}

To a family $\left(x_{i}\right)_{i \in I}$, we associate the free Lie algebra $\operatorname{Lib}\left(\left(x_{i}\right)_{i \in I}\right)$ generated over $\mathbf{C}$. We will denote $\operatorname{Lib}^{\wedge}\left(\left(x_{i}\right)_{i \in I}\right)$ the completion of $\operatorname{Lib}\left(\left(x_{i}\right)_{i \in I}\right)$ for the descending central filtration:

$$
L^{\wedge}:=\operatorname{Lib}^{\wedge}\left(\left(x_{i}\right)_{i \in I}\right)=\lim _{n \in \mathbf{N}} L / L^{n},
$$

with $L:=\operatorname{Lib}\left(\left(x_{i}\right)_{i \in I}\right)$ and $L^{1}:=L, L^{n+1}:=\left[L, L^{n}\right]$.

If $I$ is finite, we refer to [6] for the following properties. Then each $L / L^{n}$ is a finite dimensional nilpotent complex Lie algebra, therefore it is an algebraic Lie algebra and $L$ is a pronilpotent proalgebraic Lie algebra.

The functor "Lie algebra" is an equivalence between the category of unipotent algebraic groups and the category of finite dimensional nilpotent Lie algebras. We shall denote exp the inverse equivalence.

We set:

$$
\exp \left(L^{\wedge}\right):=\lim _{n \in \mathbf{N}} \exp \left(L / L^{n}\right)
$$

It is a prounipotent algebraic group, whose Lie algebra is $L \hat{\text {. }}$

If $I$ is infinite, then the situation is more complicated. The dimension of each nilpotent Lie algebra $L / L^{n}$ is infinite and the pronilpotent completion 
$L^{\wedge}$ is not satisfying for our purposes. Therefore we will introduce another completion of $L$, the f-pronilpotent completion $L^{\dagger}$.

Let $J \subset I$ be a finite subset. We have a natural map of Lie algebras:

$$
p_{J}: \operatorname{Lib}\left(\left(x_{i}\right)_{i \in I}\right) \rightarrow \operatorname{Lib}\left(\left(x_{j}\right)_{j \in J}\right),
$$

defined by $p_{J}\left(x_{i}\right):=0$ if $i \notin J$ and $p_{J}\left(x_{i}\right):=x_{i}$ if $i \in J$. We define similarly maps $p_{J_{1}, J_{2}}: \operatorname{Lib}\left(\left(x_{i}\right)_{i \in J_{2}}\right) \rightarrow \operatorname{Lib}\left(\left(x_{j}\right)_{j \in J_{1}}\right)$ if $J_{1} \subset J_{2} \subset I$ ( $J_{2}$ finite).

Going to the nilpotent completions, we get maps:

$$
\hat{p}_{J}: \operatorname{Lib}^{\wedge}\left(\left(x_{i}\right)_{i \in I}\right) \rightarrow \operatorname{Lib}^{\wedge}\left(\left(x_{j}\right)_{j \in J}\right), \quad p_{J_{1}, J_{2}}: \operatorname{Lib}^{\wedge}\left(\left(x_{i}\right)_{i \in J_{2}}\right) \rightarrow \operatorname{Lib}^{\wedge}\left(\left(x_{j}\right)_{j \in J_{1}}\right)
$$

The $\operatorname{Lib}^{\wedge}\left(\left(x_{j}\right)_{j \in J}\right)(J \subset I, J$ finite $)$ are pronilpotent proalgebraic Lie algebras and the $p_{J_{1}, J_{2}}\left(J_{2} \subset I\right.$ finite, $\left.J 1 \subset J_{2}\right)$ are morphisms of proalgebraic Lie algebras.

We thus get a projective system of prounipotent proalgebraic Lie algebras and, by definition, the f-pronilpotent completion $L^{\dagger}:=\operatorname{Lib}^{\dagger}\left(\left(x_{i}\right)_{i \in I}\right)$ of $L:=$ $\operatorname{Lib}\left(\left(x_{i}\right)_{i \in I}\right)$ is the projective limit of this system,

$$
L^{\dagger}:=\lim _{\overleftarrow{J \subset I}} \operatorname{Lib}^{\wedge}\left(\left(x_{j}\right)_{j \in J}\right), \quad J \text { finite. }
$$

It can be interpreted as a projective limit of prounipotent proalgebraic Lie algebras. Then we can pass to groups, using the functor exp, and we can define a projective limit of unipotent groups $\exp L^{\dagger}$, whose Lie algebra is $L^{\dagger}$.

The natural map $L \rightarrow L^{\dagger}$ is injective and dominant (its image is dense).

Remark A.1. - If $I$ is finite, then $L^{\dagger}=L^{\wedge}$.

If $I$ is infinite, then we have maps $L \rightarrow L^{\wedge} \rightarrow L^{\dagger}$ and $L^{\wedge} \rightarrow L^{\dagger}$ is not an isomorphism.

We shall consider now some actions of an abelian proalgebraic group $G$ on a free Lie algebra $L$ and the corresponding "semi-direct products" $L \rtimes G$.

In what follows we will suppose that each one dimensional complex vector space $\mathbf{C} x_{i}$ is stable under the action of $G$ and that the action of $G$ on $\mathbf{C} x_{i}$ is, for all $i \in I$, algebraic. Therefore the representations $\rho_{i}: G \rightarrow \mathbf{C}^{*}$, given by $g \in G \mapsto \rho_{i}(g)$, with $g\left(x_{i}\right)=\rho_{i}(g) x_{i}$ are rational, they are weights on $G$.

By definition a representation $\rho$ of $L \rtimes G$ is the data of a rational linear representation $\rho^{\prime}$ of $G\left(\rho^{\prime}: G \rightarrow \mathrm{GL}(V)\right)$, together with a representation $d \rho^{\prime \prime}$ of $L$ in the same space $\left(d \rho^{\prime \prime}: L \rightarrow \operatorname{End}(V)\right)$, required to be compatible with the corresponding adjoint actions. We consider the corresponding Tannakian 
category $\operatorname{Rep}(L \rtimes G)$.

In what follows we will suppose that:

(i) for all weight on $G$, there exists only a finite set of $i \in I$ such that $\rho_{i}=\rho$;

(ii) for every representation $\rho=\left(\rho^{\prime}, d \rho^{\prime \prime}\right)$ of $L \rtimes G$, the image of $d \rho^{\prime \prime}$ is a nilpotent subalgebra of $\operatorname{End}(V)$.

Lemma A.2. - Let $\rho=\left(\rho^{\prime}, d \rho^{\prime \prime}\right)$ be a representation of $L \rtimes G$. Then there exists only a finite set of $i \in I$ such that $d \rho^{\prime \prime}\left(x_{i}\right) \neq 0$.

Proof. - Let $\rho=\left(\rho^{\prime}, d \rho^{\prime \prime}\right)$ be a representation of $L \rtimes G$ in a finite dimensional space $V$. Let $i \in I$, for all $g \in G$ :

$$
\operatorname{Ad}_{\rho^{\prime}(g)}\left(d \rho^{\prime \prime}\left(x_{i}\right)\right)=d \rho^{\prime \prime}\left(g\left(x_{i}\right)\right)=d \rho^{\prime \prime}\left(\rho_{i}(g) x_{i}\right)=\rho_{i}(g) d \rho^{\prime \prime}\left(x_{i}\right) .
$$

We suppose that $d \rho^{\prime \prime}\left(x_{i}\right) \neq 0$. There exists $g_{0} \in G$ such that $\rho_{i}\left(g_{0}\right) \neq 1$, then $\operatorname{Ad}_{\rho^{\prime}\left(g_{0}\right)}\left(d \rho^{\prime \prime}\left(x_{i}\right)\right)=\rho_{i}\left(g_{0}\right) d \rho^{\prime \prime}\left(x_{i}\right)$, therefore there exists a root $\xi$ for the adjoint action of $\rho_{1}(G)$ on $\operatorname{End} V$ such that $d \rho^{\prime \prime}\left(x_{i}\right)$ belongs to the corresponding root space and we have $\rho_{i}=\xi \circ \rho^{\prime}$. The number of roots $\xi$ is finite, the result follows, using the condition (i).

If $J \subset I$ is a finite subset such that, for all $i \in I \backslash J, d \rho^{\prime \prime}\left(x_{i}\right)=0$, then the representation $d \rho^{\prime \prime}$ factors by $\operatorname{Lib}\left(\left(x_{j}\right)_{j \in J}\right)$ and, as the image of $d \rho^{\prime \prime}$ is nilpotent, it factors by $\operatorname{Lib}^{\wedge}\left(\left(x_{j}\right)_{j \in J}\right)$. Therefore the natural map:

$$
\operatorname{Lib}\left(\left(x_{i}\right)_{i \in I}\right) \rightarrow \operatorname{Lib}^{\dagger}\left(\left(x_{i}\right)_{i \in I}\right),
$$

induces an isomorphism:

$$
\operatorname{Rep}\left(\operatorname{Lib}^{\dagger}\left(\left(x_{i}\right)_{i \in I}\right) \rtimes G\right) \rightarrow \operatorname{Rep}\left(\operatorname{Lib}\left(\left(x_{i}\right)_{i \in I}\right) \rtimes G\right) .
$$

Proposition A.3. - Under the above conditions, the tannakian group of the tannakian category $\operatorname{Rep}\left(\operatorname{Lib}\left(\left(x_{i}\right)_{i \in I}\right) \rtimes G\right)$ is isomorphic to Lib ${ }^{\dagger}\left(\left(x_{i}\right)_{i \in I}\right) \rtimes G$. More precisely, if we have a $G$-equivariant morphism of prounipotent proalgebraic Lie algebras $\varphi: \operatorname{Lib}^{\dagger}\left(\left(x_{i}\right)_{i \in I} \rightarrow \Lambda\right.$ inducing an isomorphism:

$$
\operatorname{Rep}(\Lambda \rtimes G) \rightarrow \operatorname{Rep}\left(\operatorname{Lib}^{\dagger}\left(\left(x_{i}\right)_{i \in I}\right) \rtimes G\right),
$$

then $\varphi$ is an isomorphism. 
Example A.4. - Our main example is:

$$
I:=\left\{\iota=(\delta, \bar{c}, i) \mid(\delta, \bar{c}) \in \mathbf{N}^{*} \times \mathbf{E}_{q}, i=1, \ldots, \delta\right\} \cup\{0\},
$$

with $x_{\iota}:=\dot{\Delta}_{i}^{(\delta, \bar{c})}$ if $\iota \neq 0$ and $x_{0}:=\dot{\Delta}^{(0)}$. Then $L:=\operatorname{Lib}\left(\left(x_{\iota}\right)_{\iota \in I}\right), G:=G_{p, 1, s}^{(0)}$. The weight $\rho_{i}$ are defined by:

$$
\rho_{\iota}:=\delta \bar{c},
$$

$\mathbf{E}_{q}$ being interpreted as the group of weights on $\left.\operatorname{Hom}_{g r}\left(\mathbf{E}_{q}, \mathbf{C}^{*}\right)\right)$ if $\iota \neq 0$ and $\rho_{1}:=1$.

It is easy ro check that the conditions (i), (ii) are satisfied.

Using the proposition A.3, we prove that

$$
L^{\dagger} \rightarrow \tilde{\mathfrak{s t}}
$$

is an isomorphism of pronilpotent proalgebraic Lie algebras. it follows that

$$
\exp \left(L^{\dagger}\right) \rtimes G_{p, 1, s}^{(0)} \rightarrow \exp (\tilde{\mathfrak{s t}}) \rtimes G_{p, 1, s}^{(0)}=G_{1}^{(0)}
$$

is an isomorphism of proalgebraic groups, giving a transcendental explicit description of the $q$-difference universal local Galois group $G_{1}^{(0)}$.

A.0.0.1. Variations. - It is possible to use (more complicated) variants of the above formalism for various problems of local classification of dynamical systems.

1. Local classification of meromorphic linear differential equations. In that case condition (ii) is not satisfied.

2. Local classification of meromorphic linear difference equations.

3. Local classification of meromorphic saddle nodes in the plane. In that case it is necessary to use some infinite dimensional representations. As an exercise the reader can explicit this example using the dictionnary between the Martinet-Ramis classification and the Ecalle resurgent classification detailed in 33 .

There are also some analogies with the wild ramification phenomena in the classical Galois theory of local fields, but that is another story.

\section{References}

[1] Yves André. Idées galoisiennes. 2012.

[2] Frits Beukers, W.Dale Brownawell, and Gert Heckman. Siegel normality. Annals of Mathematics, (127):279-308, 1988.

[3] George D. Birkhoff and Paul E. Guenther. Note on a canonical form for the linear q-difference system. Proc. Nat. Acad. Sci. U. S. A., 27:218-222, 1941. 
[4] Nicolas Bourbaki. Elements of mathematics. Lie groups and Lie algebras. Chapters 2 and 3. (Éléments de mathématique. Groupes et algèbres de Lie. Chapitres 2 et 3.) Reprint of the 1972 original. Berlin: Springer. 320 p., 2006.

[5] Alain Connes and Matilde Marcolli. Renormalization and motivic Galois theory. Int. Math. Res. Not., 2004(76):4073-4091, 2004.

[6] P. Deligne and A.B. Goncharov. Groupes fondamentaux motiviques de tate mixtes. Ann. Sci. École Norm. Sup. (4), 38(1):1-56, 2005.

[7] Pierre Deligne, Bernard Malgrange, and Jean-Pierre Ramis. Irregular singularities. Correspondence and documents. (Singularités irrégulières. Correspondance et documents.). Documents Mathématiques (Paris) 5. Paris: Société Mathématique de France. x, 188 p. EUR 47.00 , 2007.

[8] Pierre Deligne and J.S. Milne. Tannakian categories. Hodge cycles, motives, and Shimura varieties, Lect. Notes Math. 900, 101-228 (1982)., 1982.

[9] Anne Duval and Claude Mitschi. Matrices de Stokes et groupe de Galois des équations hypergéometriques confluentes generalisées. (Stokes matrices and Galois groups of generalized confluent hypergeometric equations). Pac. J. Math., 138(1):25$56,1989$.

[10] Pavel Etingof. Galois groups and connection matrices for $q$-difference equations. Electron. Res. Announc. Am. Math. Soc., 1(1):1-9, 1995.

[11] Jean Frenkel. Cohomologie non abélienne et espaces fibrés. Bull. Soc. Math. Fr., 85:135-220, 1957.

[12] James E. Humphreys. Linear algebraic groups. Corr. 2nd printing. Graduate Texts in Mathematics, 21. New York - Heidelberg - Berlin: Springer-Verlag., 1981.

[13] Nicholas M. Katz. On the calculation of some differential Galois groups. Invent. Math., 87:13-61, 1987.

[14] E.R. Kolchin. Algebraic matric groups and the Picard-Vessiot theory of homogeneous linear ordinary differential equations. Annals of Math., 49(2):1-42, 1948.

[15] Michèle Loday-Richaud and Pascal Remy. Resurgence, Stokes phenomenon and alien derivatives for level-one linear differential systems. J. Differ. Equations, 250(3):1591-1630, 2011.

[16] Jean Martinet and Jean-Pierre Ramis. Théorie de Galois différentielle et resommation. (Differential Galois theory and resummation.). Computer algebra and differential equations, Colloq., Comput. Math. Appl., 117-214 (1988)., 1988.

[17] Jean Martinet and Jean-Pierre Ramis. Elementary acceleration and multisummability. I. Ann. Inst. Henri Poincaré Phys. Théorique, 54(4):331-401, 1991.

[18] Claude Mitschi. Differential Galois groups of confluent generalized hypergeometric equations: An approach using Stokes multipliers. Pac. J. Math., 176(2):365-405, 1996.

[19] G.D. Mostow. Fully reducible subgroups of algebraic groups. Am. J. Math., 78:200-221, 1956.

[20] J.-P. Ramis and J. Sauloy. The $q$-analogue of the wild fundamental group. I. In Algebraic, analytic and geometric aspects of complex differential equations and their deformations. Painlevé hierarchies, RIMS Kôkyûroku Bessatsu, B2, pages 167-193. Res. Inst. Math. Sci. (RIMS), Kyoto, 2007. 
[21] Jean-Pierre Ramis and Jacques Sauloy. The $q$-analogue of the wild fundamental group. II. In Differential Equations and Singularities, 60th years of J.M. Aroca, volume 323. Société Mathématique de France, 2009.

[22] Jean-Pierre Ramis, Jacques Sauloy, and Changgui Zhang. Local analytic classification of irregular q-difference equations. Accepted for publication by Astérisque; meanwhile, see [23, 24] and http://front.math.ucdavis.edu/0903.0853

[23] Jean-Pierre Ramis, Jacques Sauloy, and Changgui Zhang. La variété des classes analytiques d'équations aux $q$-différences dans une classe formelle. C. R. Math. Acad. Sci. Paris, 338(4):277-280, 2004.

[24] Jean-Pierre Ramis, Jacques Sauloy, and Changgui Zhang. Développement asymptotique et sommabilité des solutions des équations linéaires aux $q$-différences. C. R. Math. Acad. Sci. Paris, 342(7):515-518, 2006.

[25] Julien Roques. On classical irregular q-difference equations. Composition Mathematica (to appear).

[26] Julien Roques. Galois groups of the basic hypergeometric equations. Pac. J. Math., 235(2):303-322, 2008.

[27] Julien Roques. Generalized basic hypergeometric equations. Invent. Math., 184(3):499-528, 2011.

[28] Neantro Saavedra Rivano. Catégories tannakiennes. Lecture Notes in Mathematics. 265. Berlin-Heidelberg-New York: Springer-Verlag., 1972.

[29] Jacques Sauloy. Systèmes aux q-différences singuliers réguliers: classification, matrice de connexion et monodromie. Ann. Inst. Fourier (Grenoble), 50(4):10211071, 2000.

[30] Jacques Sauloy. Galois theory of Fuchsian q-difference equations. Ann. Sci. École Norm. Sup. (4), 36(6):925-968 (2004), 2003.

[31] Jacques Sauloy. Algebraic construction of the Stokes sheaf for irregular linear q-difference equations. Astérisque, 296:227-251, 2004. Analyse complexe, systèmes dynamiques, sommabilité des séries divergentes et théories galoisiennes. I.

[32] Jacques Sauloy. La filtration canonique par les pentes d'un module aux $q$ différences et le gradué associé. Ann. Inst. Fourier (Grenoble), 54(1):181-210, 2004.

[33] David Sauzin. Mould expansions for the saddle-node and resurgence monomials. Connes, Alain (ed.) et al., Renormalization and Galois theories. Selected papers of the CIRM workshop, Luminy, France, March 2006. Zürich: European Mathematical Society. IRMA Lectures in Mathematics and Theoretical Physics 15, 83-163 (2009)., 2009.

[34] Carol Tretkoff and Marvin Tretkoff. Solution of the inverse problem of differential Galois theory in the classical case. Am. J. Math., 101:1327-1332, 1979.

[35] Marius van der Put and Marc Reversat. Galois theory of $q$-difference equations. Ann. Fac. Sci. Toulouse Math. (6), 16(3):665-718, 2007.

[36] Marius van der Put and Michael F. Singer. Galois theory of linear differential equations. Grundlehren der Mathematischen Wissenschaften 328. Berlin: Springer., 2003. 
Jean-Pierre Ramis, Institut de France (Académie des Sciences) and Institut de Mathématiques, CNRS UMR 5219, Équipe Émile Picard, U.F.R. M.I.G., Université Paul Sabatier (Toulouse 3), 31062 Toulouse CEDEX 9

E-mail : ramis.jean-pierre@wanadoo.fr

JACQUES SAuloy, Institut de Mathématiques, CNRS UMR 5219, Équipe Émile

Picard, U.F.R. M.I.G., Université Paul Sabatier (Toulouse 3), 31062

Toulouse CEDEX 9 • E-mail : sauloy@math.univ-toulouse.fr

Url : www.math.univ-toulouse.fr/ $/$ sauloy/ 